\title{
From Heun Class Equations to Painlevé Equations
}

Jan DEREZIŃSKI a ${ }^{\text {, Artur ISHKHANYAN }}{ }^{\text {bc }}$ and Adam LATOSIŃSKI ${ }^{\mathrm{a}}$

a) Department of Mathematical Methods in Physics, Faculty of Physics, University of Warsaw, Pasteura 5, 02-093, Warszawa, Poland

E-mail: jan.derezinski@fuw.edu.pl,adam.latosinski@fuw.edu.pl

b) Russian-Armenian University, 0051 Yerevan, Armenia

E-mail: aishkhanyan@gmail.com

c) Institute for Physical Research of NAS of Armenia, 0203 Ashtarak, Armenia

Received August 25, 2020, in final form May 25, 2021; Published online June 07, 2021

https://doi.org/10.3842/SIGMA.2021.056

\begin{abstract}
In the first part of our paper we discuss linear 2nd order differential equations in the complex domain, especially Heun class equations, that is, the Heun equation and its confluent cases. The second part of our paper is devoted to Painlevé I-VI equations. Our philosophy is to treat these families of equations in a unified way. This philosophy works especially well for Heun class equations. We discuss its classification into 5 supertypes, subdivided into 10 types (not counting trivial cases). We also introduce in a unified way deformed Heun class equations, which contain an additional nonlogarithmic singularity. We show that there is a direct relationship between deformed Heun class equations and all Painlevé equations. In particular, Painlevé equations can be also divided into 5 supertypes, and subdivided into 10 types. This relationship is not so easy to describe in a completely unified way, because the choice of the "time variable" may depend on the type. We describe unified treatments for several possible "time variables".
\end{abstract}

Key words: linear ordinary differential equation; Heun class equations; isomonodromy deformations; Painlevé equations

2020 Mathematics Subject Classification: 34A30; 34B30; 34M55; 34M56

\section{Contents}

1 Introduction $\quad 2$

2 Second order linear differential equations in the complex domain 6

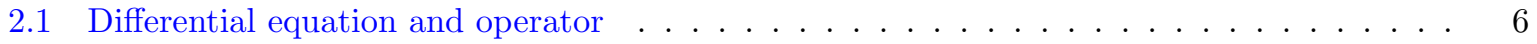

2.2 Singularities of functions $\ldots \ldots \ldots \ldots \ldots \ldots \ldots \ldots$

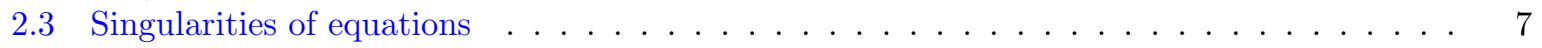

2.4 Sandwiching with functions $\ldots \ldots \ldots \ldots \ldots \ldots \ldots$

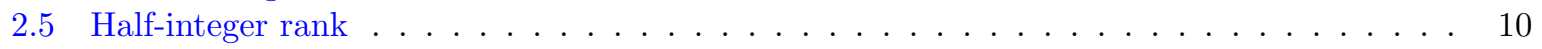

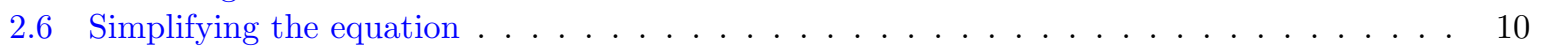

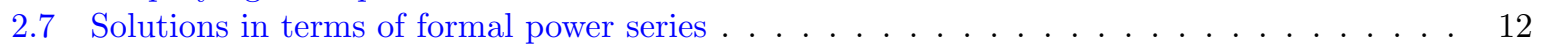

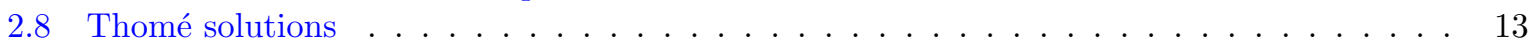

2.9 Nonlogarithmic singularities . . . . . . . . . . . . . . . . . 16

3 Equations with rational coefficients $\quad 17$

3.1 The $M_{n}$ class and the grounded $M_{n}$ class $\ldots \ldots \ldots \ldots \ldots$

3.2 Generalized Fuchs relation . . . . . . . . . . . . . . . . . . . . . . . . . . . 19

3.3 Riemann class equations . . . . . . . . . . . . . . . . . . . . . . . . . . . . . . 19

3.4 Heun class equations . . . . . . . . . . . . . . . . . . . . . . . . 20

3.5 Deformed Heun class equations . . . . . . . . . . . . . . . . . . . . 21

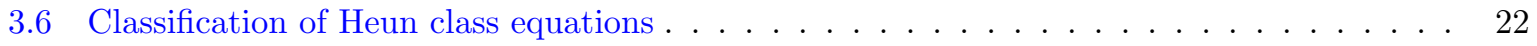


4 From Heun class to Painlevé equations $\quad 24$

4.1 Method of isomonodromic deformations . . . . . . . . . . . . . . . 24

4.2 Isomonodromic deformations in presence of a non-logarithmic singularity $\ldots \ldots \ldots$

4.3 Isomonodromic deformations of Heun class equations . . . . . . . . . . . . . . . . 26

4.4 Correspondence between Heun class and Painlevé equations . . . . . . . . . . . . . . . 30

4.5 From Heun $(111 ; 1)$ to Painlevé VI . . . . . . . . . . . . . . . . . . . 32

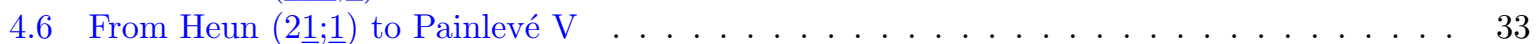

4.7 From Heun $\left(\frac{3}{2} \underline{1} ; \underline{1}\right)$ to degenerate Painlevé V . . . . . . . . . . . . . . . . . 34

4.8 From Heun $(2 ; 2)$ to non-degenerate Painlevé III' $\ldots \ldots \ldots \ldots$. . . . . . . . . 34

4.9 From Heun $\left(2 ; \frac{3}{2}\right)$ to degenerate Painlevé III' $\ldots \ldots \ldots \ldots$

4.10 From Heun $\left(\frac{3}{2} ; 2\right)$ to degenerate Painlevé III' $\ldots \ldots \ldots \ldots \ldots$

4.11 From Heun $\left(\frac{3}{2} ; \frac{3}{2}\right)$ to doubly degenerate Painlevé III' $\ldots \ldots \ldots$. . . . . . . . . . 36

4.12 From Heun $(1 ; 3)$ to Painlevé IV . . . . . . . . . . . . . . . . . . . . 37

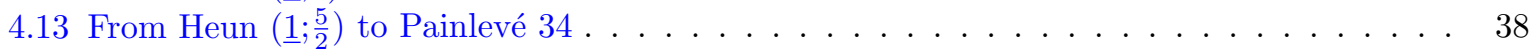

4.14 From Heun $(; 4)$ to Painlevé II . . . . . . . . . . . . . . . . . . . . . . 38

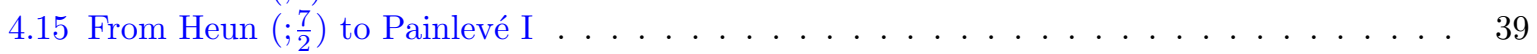

5 Five supertypes of Painlevé equation 39

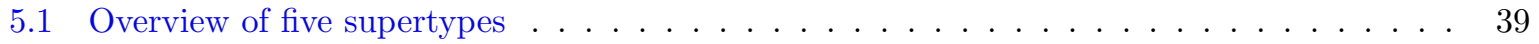

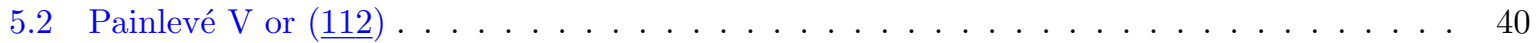

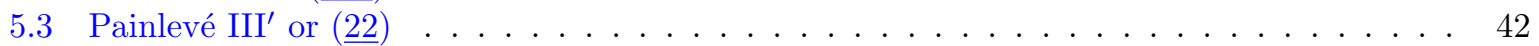

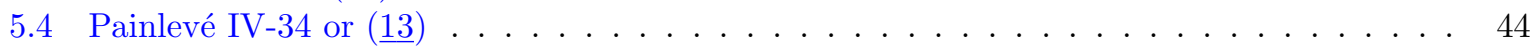

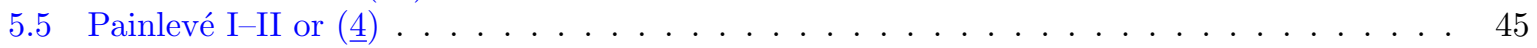

$\begin{array}{ll}\text { A Proof of Theorems 4.1, 4.2 and 4.3 } & 47\end{array}$

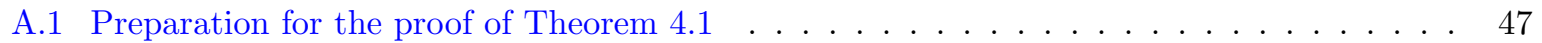

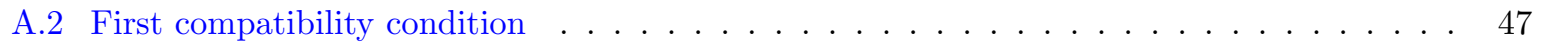

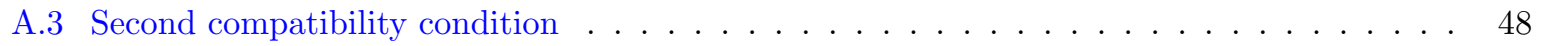

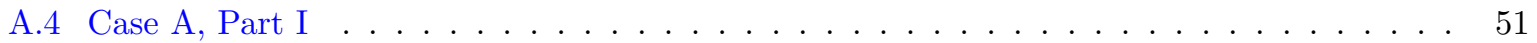

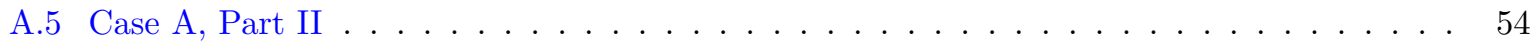

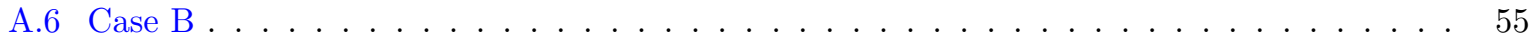

B Hamilton equations $\quad \mathbf{5 6}$

B.1 From Hamilton equations to second order equations . . . . . . . . . . . . . . 56

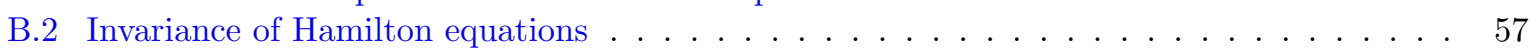

B.3 Hamiltonian solvable in quadratures . . . . . . . . . . . . . . . . . 57

$\begin{array}{ll}\text { References } & 58\end{array}$

\section{Introduction}

There are many types of differential equations and special functions. Typically, within a given class there is one generic type and many confluent types. This is the case of Riemann (hypergeometric) class equations, Heun class equations, as well as Painlevé equations. For instance, the generic type of Riemann class equations can always be reduced to the Gauss hypergeometric equation, but there are also confluent types such as Kummer's confluent equation, the $F_{1}$ equation, and the Hermite equation, see, e.g., [2, 3, 17, 24, 25].

One can try to understand the process of confluence by considering equations depending holomorphically on parameters. Some properties of the whole class can be described in a uniform way, without splitting the class into types. For instance, one can identify various transformations ("symmetries") that leave the class invariant.

In order to study the equations in more detail, one needs to split the class into types. Within a given type one can simplify the equation by symmetries and convert it to a normal form, thereby reducing the number of parameters. This has to be done case by case. 
In the case of hypergeometric class equations, this idea was successfully applied in the book by Nikiforov-Uvarov [18]. It works especially well for hypergeometric polynomials, that is Jacobi, Laguerre, Bessel and Hermite polynomials, which can be elegantly treated in a unified way.

In this paper, we try to apply this idea to the derivation of Painlevé equations from Heun class equations by the method of isomonodromic deformation. We will see that each type of Heun class equation corresponds to a (properly understood) type of Painlevé equation. The passage from Heun class to Painlevé can be accomplished in a fairly uniform way, although one has to consider several similar but distinct cases.

We start our paper by Section 2 containing basic theory of singularities of 2 nd order scalar ordinary differential equations. This is a classic subject with several well-known textbooks, such as $[9,23]$. We follow to a large extent the treatment described in the monograph by SlavyanovLay [25] and the appendix to [24], written by Slavyanov. Sometimes we introduce new notation and terminology to make precise some concepts which in [25] are implicit.

The central concept of the theory of linear differential equations in the complex domain is the rank of a singularity. In our paper we introduce several kinds of the rank. In particular, we distinguish between the usual rank and the absolute rank (the infimum over the ranks of all possible transformed forms of a given equation). The rank can be an integer or a half-integer. We also introduce the rounded rank, which has always an integer value. Thus if the rank is $m$ or $m-\frac{1}{2}$, where $m$ is an integer, then we say that its rounded rank is $m$. In particular, the rounded rank is 1 if the singularity is Fuchsian (also called regular). We believe that all these concepts clarify the theory of ordinary differential equations. We also discuss formal power series solutions of these equations (the so-called Thomé solutions). We introduce the concept of indices of a singular point. This is of course well-known for Fuchsian singularities. For non-Fuchsian singularities this concept is not so well known, although it is implicit in [25].

In Section 3 we discuss equations with rational coefficients. Such equations have a finite number of singularities on the Riemann sphere. Following [25], the class of equations with $n$ singularities in $\mathbb{C}$ and a singularity at infinity, and their confluent cases are called $M_{n}$ class equations. red It is easy to see that these equations can be written as

$$
\left(\sigma(z) \partial_{z}^{2}+\tau(z) \partial_{z}+\eta(z)\right) u(z)=0
$$

where $\sigma$ is a polynomial of degree $\leq n, \tau$ of degree $\leq n-1$ and $\sigma \eta$ of degree $\leq 2 n-2$. We introduce also a closely related grounded $M_{n}$ class, for which one of indices of all finite singularities is zero - hence the name "grounded". (In [25] such equations are called canonical. In our opinion, the word canonical is overused, hence not appropriate for this meaning.) They can be written as (1.1) with the same conditions on $\sigma, \tau$, and with $\eta$ a polynomial of degree $\leq n-2$.

The best known classes of equations with rational coefficients are the $M_{2}$ class and the grounded $M_{2}$ class. We call $M_{2}$ the Riemann class, since its generic representative is the Riemann equation with one singularity at $\infty$. The grounded $M_{2}$ class is especially often encountered in the literature. It is the main subject of the textbook by Nikiforov-Uvarov [18], where its elements are called "hypergeometric type equations". Note that the difference between the full and grounded Riemann class is minor - the only type of equations contained in the full Riemann class but not represented in the grounded Riemann class is the Airy equation.

One of the central objects of our paper is the $M_{3}$ class, called also the Heun class, for which $\sigma$ is a polynomial of degree $\leq 3, \tau$ of degree $\leq 2$ and $\sigma \eta$ of degree $\leq 4$. The main type within the Heun class is the standard Heun type, that is the equation with 4 Fuchsian singularities in the Riemann sphere, one of which put at $\infty$. It was first analyzed by Heun [8], see also [16] for a recent study.

In the literature the name Heun class equations is employed in two meanings: the meaning that we have just described is used in $[24,25]$. It is also common to use it for what we call the 
grounded Heun class, see, e.g., [5]. The grounded Heun class (not counting types of the Riemann class) is divided into 5 types: standard, confluent, biconfluent, doubly confluent and triconfluent. The full Heun class, beside the above five types has five more types, which we call degenerate confluent, degenerate biconfluent, degenerate doubly confluent, doubly degenerate doubly confluent and degenerate triconfluent. ([25] uses the word "reduced" instead of "degenerate".)

Sometimes a singularity of an equation does not lead to a singularity of its solutions. We call such points non-logarithmic singularities. Non-logarithmic singularities play an important role in the following construction.

Let us start with an equation (1.1). We introduce the deformed form of (1.1) to be the equation

$$
\begin{aligned}
& \left(\sigma(z) \partial_{z}^{2}+\left(\tau(z)-\frac{\sigma(z)}{z-\lambda}\right) \partial_{z}+\eta(z)-\eta(\lambda)-\mu^{2} \sigma(\lambda)-\mu\left(\tau(\lambda)-\sigma^{\prime}(\lambda)\right)+\frac{\mu \sigma(\lambda)}{z-\lambda}\right) \\
& \quad \times v(z)=0 .
\end{aligned}
$$

Note that all finite singularities of (1.2) are the same as in (1.1), except that there is an additional non-logarithmic singularity with indices $0,2 . \lambda$ is the position of this singularity and $\mu=\frac{v^{\prime}}{v}(\lambda)$. If (1.1) is a Heun class equation, then (1.2) is called a deformed Heun class equation [25].

Let us note that non-logarithmic singularities of an equation can be produced by a change of gauge, that is by replacing the unknown $v(z)$ with $c_{0}(z) v(z)+c_{1}(z) v^{\prime}(z)$, where $c_{0}, c_{1}$ are some rational functions. This procedure applied to grounded Heun class equations produces the so-called derivative Heun class equations, see [5, 10, 26]. Kimura proved in [15] that in deformed Riemann class equations one can remove all non-logarithmic singularities by a change of gauge. We do not know if the same is possible for all kinds of deformed Heun class equations.

Painlevé equations is a famous class of nonlinear differential equations with the so called Painlevé property-the absence of moving essential and branch singularities in its solutions. They were discovered in the beginning of 20th century [7, 21, 22]. Traditionally, Painlevé equations are divided into 6 types, called Painlevé I, II, III, IV, V and VI [12]. As noted by OhyamaOkumura [19], it is actually natural to subdivide some of them into smaller types, obtaining altogether 10 types. Each of them corresponds to one of types of the Heun class equations. We obtain the following correspondence between types of the Heun class and Painlevé:

\begin{tabular}{cll}
\hline$(\underline{1111})$ & (standard) Heun & Painlevé VI \\
\hline$(\underline{112})$ & confluent Heun & Painlevé nondegenerate V \\
\hline$\left(\underline{11} \frac{3}{2}\right)$ & degenerate confluent Heun & Painlevé degenerate V \\
\hline$(22)$ & doubly confluent Heun & Painlevé nondegenerate III' \\
\hline$\left(\frac{3}{2} 2\right)$ & degenerate doubly confluent Heun & Painlevé degenerate III' $^{\prime}$ \\
\hline$\left(\frac{3}{2} \frac{3}{2}\right)$ & doubly degenerate doubly confluent Heun & Painlevé doubly degenerate III' \\
\hline$(\underline{13})$ & bi-confluent Heun & Painlevé IV \\
\hline$\left(\underline{1} \frac{5}{2}\right)$ & degenerate bi-confluent Heun & Painlevé 34 \\
\hline$(4)$ & tri-confluent Heun & Painlevé II \\
\hline$\left(\frac{7}{2}\right)$ & degenerate tri-confluent Heun & Painlevé I \\
\hline
\end{tabular}

Above, the symbols such as (112) indicate the ranks of the singularities in the Heun class equation. We use underline to indicate the rounded rank: thus
1 denotes either 1 or $\frac{1}{2}$ (a Fuchsian singularity);
2 denotes either 2 or $\frac{3}{2}$;
$\underline{3}$ denotes either 3 or $\frac{5}{2}$;
4 denotes either 4 or $\frac{7}{2}$. 
Following the literature, instead of Painlevé III we prefer to use an equivalent equation Painlevé III'.

Note that the Painlevé deg-V is equivalent to Painlevé $\mathrm{III}^{\prime}$ and Painlevé 34 is equivalent to Painlevé II by a relatively complicated change of variables.

As noted by Ohyama-Okumura [19], there also exists another, coarser classification of Painlevé equations into five supertypes. It corresponds to a coarser classification of Heun class equations into supertypes, where we use rounded ranks instead of the usual ranks. We obtain the following table, which is discussed in detail in Section 5:

\begin{tabular}{cll}
\hline$(\underline{1111})$ & (standard) Heun & Painlevé VI \\
\hline$(\underline{112})$ & confluent Heun & Painlevé V \\
\hline$(\underline{22})$ & doubly confluent Heun & Painlevé III' \\
\hline$(\underline{13})$ & bi-confluent Heun & Painlevé IV-34 \\
\hline$(\underline{4})$ & tri-confluent Heun & Painlevé II-I \\
\hline
\end{tabular}

The main topic of our paper, described in Section 4, is a derivation of Painlevé equations from Heun class equations. The first step of this derivation is a choice of a family of Heun class equations depending on a parameter denoted $t$ and called the time. Then we consider then the corresponding deformed Heun class equation, (1.2), which depends on two additional variables: $\lambda, \mu$. The conditions for a constant monodromy lead to a set of nonlinear differential equation for $\lambda, \mu$ in terms of $t$. These equations can be interpreted as Hamilton equations generated by $H(t, \lambda, \mu)$, which we will call Painlevé Hamiltonians.

The most difficult ingredient of the passage from Heun class to Painlevé is the choice of the time variable and of the so-called compatibility functions $a, b$, which control the isomonodromic deformations. The main idea of our paper is to present this passage in a unified way. Our first attempt in this direction is described in Theorem 4.1. The description of compatibility functions and the corresponding Hamiltonian contained in this theorem is unified, however it is not very transparent. Theorem 4.2, which can be viewed as the first part of the main result of this paper, is more satisfactory. The method of isomodronomic deformation is here divided into two slightly different ansatzes, called Cases $\mathrm{A}$ and $\mathrm{B}$. The main condition on $\sigma, \tau, \eta, t$ for the applicability of Ansatz A is the existence of a zero of $\sigma$, so that we can write $\sigma(z)=(z-s) \rho(z)$, where $\rho$ is a polynomial of degree $\leq 2$. Under these conditions there exists a function $t \mapsto m(t)$ such that

$$
H(t, \lambda, \mu)=m\left(\sigma(\lambda) \mu^{2}+\left(\tau(\lambda)-(\lambda-s) \rho^{\prime}(\lambda)\right) \mu+\eta(\lambda)\right)
$$

is a Painlevé Hamiltonian.

Among the conditions on $\sigma, \tau, \eta, t$ needed for Ansatz B the most important is $\operatorname{deg} \sigma \leq 2$. Then we can show that there exists $t \mapsto m(t)$ such that

$$
H(t, \lambda, \mu)=m\left(\sigma(\lambda) \mu^{2}+\left(\tau(\lambda)-\sigma^{\prime}(\lambda)\right) \mu+\eta(\lambda)\right)
$$

is a Painlevé Hamiltonian.

It seems impossible to implement Theorem 4.2 in a fully unified way, and one has to subdivide Cases A and B into several subcases. Case A splits into Subcases A1, Ap, Aq and Case B splits into Subcases Bp and Bq. We describe these subcases in Theorem 4.3. The main difference between the subcases is the choice of the time variable $t$ :

In Subcase A1 the variable $t$ is the position of one of Fuchsian singularities.

In Subcases Ap and Bp the variable $t$ is contained in $\tau$.

In Subcases $\mathrm{Aq}$ and $\mathrm{Bq}$ the variable $t$ is contained in $\eta$.

In the following list we informally describe when we can apply various subcases.

$$
\begin{aligned}
& \text { A1. } \sigma(t)=0, \sigma^{\prime}(t) \neq 0, \operatorname{deg}(z-t) \eta \leq 2 . \\
& \text { Ap. } \sigma(s)=\sigma^{\prime}(s)=0, \tau(s) \neq 0, \sigma \eta(s)=(\sigma \eta)^{\prime}(s)=0 .
\end{aligned}
$$


Aq. $\sigma(s)=\sigma^{\prime}(s)=0, \tau(s)=0, \sigma \eta(s)=0,(\sigma \eta)^{\prime}(s) \neq 0$.

Bp. $\operatorname{deg} \sigma \leq 2, \operatorname{deg} \tau=2, \operatorname{deg} \sigma \eta \leq 2$.

Bq. $\operatorname{deg} \sigma \leq 2, \operatorname{deg} \tau \leq 1, \operatorname{deg} \sigma \eta=3$.

Note that Subcase A1 works in the standard Heun type (1111), leading to Painlevé VI. Hence it works in under generic conditions. However, it does not works for some confluent types. For instance, for most degenerate types one needs to use either Subcase Aq or Subcase Bq. Altogether, Theorem 4.3 works for certain normal forms of all types of Heun class equations. This allows us to derive all types of Painlevé equations.

The derivation of the Painlevé VI equation from the Heun equation can be traced back to a paper by Fuchs [6] from the early 20th century (written by the son of Fuchs from whose name the adjective "Fuchsian" comes). The approach was generalized to other Painlevé equations by Okamoto [20] and refined by Ohyama-Okumura [19]. A discussion of the relationship between the biconfluent Heun type and the Painlevé IV equation can be also found in [1]. Thus derivations described in Sections 4.5-4.15 are known. They are in particular described by Ohyama-Okumura [19], where they were checked case by case. Our approach allows us to automatize these derivations and view them as implementations of a unified algorithm. In fact, our paper can be to some extent viewed as an explanation of the principles that underly the results of [19].

Slavyanov and Lay devote in [25] a whole chapter to the Heun class - Painlevé correspondence. In particular, they stress that Painlevé Hamiltonians can be viewed as "dequantizations" of the corresponding Heun class equations. In fact, the symbol of Heun class operators, that is the expression obtained from (1.1) by replacing $\partial_{z}$ with $\mu$ and $z$ with $\lambda$, is very similar to (1.3) and (1.4). (The difference is a "lower order term", which can be interpreted as a result of an "ordering prescription".) Nevertheless, to our understanding, [25] contains only a sketch of a program. The details of this program are quite involved and [25] does not provide its full description.

Clearly, every 2nd order scalar equation can be rewritten as a system of two 1st order equations. For instance, we can rewrite (1.1) as

$$
\sigma(z) \partial_{z}\left[\begin{array}{l}
u(z) \\
v(z)
\end{array}\right]=\left[\begin{array}{cc}
0 & 1 \\
-\sigma(z) \eta(z) & -\tau(z)+\sigma^{\prime}(z)
\end{array}\right]\left[\begin{array}{l}
u(z) \\
v(z)
\end{array}\right]
$$

(or in other ways). In particular, Heun class equations are essentially equivalent to certain systems of 1st order equations called sometimes Heun connections. This suggests a different approach to deriving Painlevé equations starting from Heun connections. This alternative approach was studied, e.g., by Jimbo and Miwa [13, 14]. A recent exposition of this approach can be found in [11].

Note that in the above references each type of Painlevé equations were treated separately. Besides, degenerate cases were usually left out. It would be interesting to investigate to what extent a derivation of all types Painlevé equations from Heun connections can be treated in a uniform way, in the spirit of Theorems 4.2 and 4.3. Anyway, in this paper we do not consider this question and we stick to (scalar 2nd order) deformed Heun class equations.

\section{Second order linear differential equations in the complex domain}

\subsection{Differential equation and operator}

Let us recall basic concepts of ordinary 2 nd order linear differential equations in the complex domain with holomorphic coefficients. They have the form (1.1), where $\sigma, \tau$ and $\eta$ are holo- 
morphic functions. We will often describe the equation (1.1) by specifying the corresponding operator

$$
\sigma(z) \partial_{z}^{2}+\tau(z) \partial_{z}+\eta(z)
$$

Clearly, by multiplying an equation from the left by an arbitrary nonzero holomorphic function we obtain an equivalent equation. However, we change the corresponding operator. Speaking of operators instead of equations, which we will often do, has two advantages. First it saves a little space, since we do not need to write the function $u$. Besides, an operator contains more information than the corresponding equation, therefore sometimes allows for making more precise statements.

Divide (1.1) and (2.1) by $\sigma(z)$ and set

$$
p(z):=\frac{\tau(z)}{\sigma(z)}, \quad q(z):=\frac{\eta(z)}{\sigma(z)} .
$$

This leads to the so-called principal form of the equation and the corresponding principal operator:

$$
\begin{aligned}
& \left(\partial_{z}^{2}+p(z) \partial_{z}+q(z)\right) u(z)=0, \\
& A:=\partial_{z}^{2}+p(z) \partial_{z}+q(z) .
\end{aligned}
$$

The principal form is often used as the standard form. However, we will often prefer different forms.

\subsection{Singularities of functions}

Let $p$ be a function holomorphic on an open subset of the Riemann sphere $\mathbb{C} \cup\{\infty\}$. Let $z_{0} \in \mathbb{C} \cup\{\infty\}$ be its singularity, so that

$$
\begin{aligned}
& p(z)=\sum_{k=-\infty}^{\infty} p_{z_{0}, k}\left(z-z_{0}\right)^{k}, \quad\left|z-z_{0}\right|<r \quad \text { for some } \quad r>0, \quad \text { if } \quad z_{0} \in \mathbb{C}, \\
& p(z)=\sum_{k=-\infty}^{\infty} p_{\infty, k} z^{k}, \quad|z|>R \quad \text { for some } \quad R \geq 0, \quad \text { if } \quad z_{0}=\infty .
\end{aligned}
$$

We define the degree of the singularity of $p$ at $z_{0}$ by

$$
\begin{aligned}
\operatorname{deg}\left(p, z_{0}\right) & :=-\min \left\{k \mid p_{z_{0}, k} \neq 0\right\}, \quad z_{0} \in \mathbb{C}, \\
\operatorname{deg}(p, \infty) & :=\max \left\{k \mid p_{\infty, k} \neq 0\right\}, \quad z_{0}=\infty .
\end{aligned}
$$

(If $p$ is a polynomial, then $\operatorname{deg}(p, \infty)=\operatorname{deg}(p)$ is its usual degree.)

Note that if $\phi$ is a biholomorphic transformation of a neighborhood of $z_{0}$ onto a neighborhood of $\phi\left(z_{0}\right)$, then

$$
\operatorname{deg}\left(p, z_{0}\right)=\operatorname{deg}\left(p \circ \phi^{-1}, \phi\left(z_{0}\right)\right) .
$$

\section{$2.3 \quad$ Singularities of equations}

Consider now the equation (2.2) (in the principal form) with holomorphic coefficients represented by the operator (2.3), which we denote by $A$. We say that $z_{0}$ is a regular point of $A$ if

$$
\begin{aligned}
& \operatorname{deg}\left(p, z_{0}\right) \leq 0, \quad \operatorname{deg}\left(q, z_{0}\right) \leq 0, \quad z_{0} \in \mathbb{C}, \\
& \operatorname{deg}\left(p-\frac{2}{z}, \infty\right) \leq-2, \quad \operatorname{deg}(q, \infty) \leq-4, \quad z_{0}=\infty .
\end{aligned}
$$

Otherwise we say that it is a singular point of $A$. 
We say that the singular point $z_{0}$ is regular or Fuchsian if

$$
\begin{array}{ll}
\operatorname{deg}\left(p, z_{0}\right) & \leq 1, \quad \operatorname{deg}\left(q, z_{0}\right) \leq 2, \quad z_{0} \in \mathbb{C}, \\
\operatorname{deg}(p, \infty) \leq-1, & \operatorname{deg}(q, \infty) \leq-2, \quad z_{0}=\infty .
\end{array}
$$

It is standard to introduce two indices of a Fuchsian singular point:

the roots of $\lambda(\lambda-1)+p_{z_{0},-1} \lambda+q_{z_{0},-2}$ are called indices of $z_{0} \in \mathbb{C}$,

the roots of $\lambda(\lambda+1)-p_{\infty,-1} \lambda+q_{\infty,-2}$ are called indices of $z_{0}=\infty$.

The rank of $A$ at $z_{0}$ is defined as follows. If $z_{0}$ is a regular point, we set $\operatorname{rk}\left(A, z_{0}\right)=0$. The case of rank equal to $\frac{1}{2}$ is somewhat special:

$$
\begin{aligned}
& \operatorname{rk}\left(A, z_{0}\right):=\frac{1}{2} \quad \text { if } \quad \operatorname{deg}\left(p-\frac{1}{2\left(z-z_{0}\right)}, z_{0}\right) \leq 0, \quad \operatorname{deg}\left(q, z_{0}\right) \leq 1, \quad z_{0} \in \mathbb{C}, \\
& \operatorname{rk}(A, \infty):=\frac{1}{2} \quad \text { if } \quad \operatorname{deg}\left(p-\frac{3}{2 z}, \infty\right) \leq-2, \quad \operatorname{deg}(q, \infty) \leq-3, \quad z_{0}=\infty
\end{aligned}
$$

If $\operatorname{rk}\left(A, z_{0}\right) \neq 0, \frac{1}{2}$, then we set

$$
\begin{aligned}
& \operatorname{rk}\left(A, z_{0}\right):=\max \left\{\operatorname{deg}\left(p, z_{0}\right), \frac{1}{2} \operatorname{deg}\left(q, z_{0}\right), 1\right\}, \quad z_{0} \in \mathbb{C}, \\
& \operatorname{rk}(A, \infty):=\max \left\{\operatorname{deg}(p, \infty)+2, \frac{1}{2} \operatorname{deg}(q, \infty)+2,1\right\}, \quad z_{0}=\infty .
\end{aligned}
$$

The rank and indices of a singularity are invariants of biholomorphic transformations. For instance, this is the case of homographies, that is $w=\frac{a z+b}{c z+d}$, or $z=\frac{d w-b}{-c w+a}$, where $a d-b c=1$. We obtain

$$
\begin{aligned}
\partial_{z}^{2}+p(z) \partial_{z}+q(z) & =(-c w+a)^{4} \partial_{w}^{2} \\
& +\left(-2 c(-c w+a)^{3}+p\left(\frac{d w-b}{-c w+a}\right)(-c w+a)^{2}\right) \partial_{w}+q\left(\frac{d w-b}{-c w+a}\right)
\end{aligned}
$$

In order to obtain the principal form we need to divide $(2.3)$ by $(-c w+a)^{4}$.

Note that the rank is always an integer or a half-integer. A singularity of rank $m-\frac{1}{2}$ with $m \in\{1,2, \ldots\}$ can be often treated as a degeneration of a singularity of rank $m$. This motivates us to introduce the rounded rank, denoted $\lceil\mathrm{rk}\rceil$ :

$$
\lceil\operatorname{rk}\rceil\left(A, z_{0}\right):= \begin{cases}\operatorname{rk}\left(A, z_{0}\right), & \text { if } \quad \operatorname{rk}\left(A, z_{0}\right) \in\{0,1,2, \ldots\}, \\ \operatorname{rk}\left(A, z_{0}\right)+\frac{1}{2}, & \text { if } \quad \operatorname{rk}\left(A, z_{0}\right) \in\left\{\frac{1}{2}, \frac{3}{2}, \frac{5}{2}, \ldots\right\} .\end{cases}
$$

Equivalently,

$$
\begin{aligned}
& \lceil\operatorname{rk}\rceil\left(A, z_{0}\right):=\left\lceil\max \left\{\operatorname{deg}\left(p, z_{0}\right), \frac{1}{2} \operatorname{deg}\left(q, z_{0}\right), 0\right\}\right], \quad z_{0} \in \mathbb{C}, \\
& \lceil\operatorname{rk}\rceil(A, \infty):=\left\lceil\max \left\{\operatorname{deg}(p, \infty)+2, \frac{1}{2} \operatorname{deg}(q, \infty)+2,0\right\}\right], \quad z_{0}=\infty
\end{aligned}
$$

Here $\lceil\cdot\rceil$ is the ceiling function, that is

$$
\lceil x\rceil:=\inf \{n \in \mathbb{Z} \mid x \leq n\} .
$$

The singularity $z_{0}$ is Fuchsian if its rank is $\frac{1}{2}$ or 1 . Thus $z_{0}$ is a Fuchsian singularity iff its rounded rank is 1 .

According to our definition, a Fuchsian singularity has rank $\frac{1}{2}$ if its indices are $0, \frac{1}{2}$. The splitting of the Fuchsian case into two subcases, the rank $\frac{1}{2}$ and 1 , is quite useful, even if its definition is not obvious. Nevertheless, in our paper we will not make much use of this splitting and both will be usually treated as one case, denoted 1 . 


\subsection{Sandwiching with functions}

The family of equations (2.2) is preserved by several kinds of transformations of the form

$$
\begin{aligned}
\mathrm{e}^{-r(z)}\left(\partial_{z}^{2}+p(z) \partial_{z}+q(z)\right) \mathrm{e}^{r(z)} & =\partial_{z}^{2}+\left(p(z)+2 r^{\prime}(z)\right) \partial_{z}+q(z)+p(z) r^{\prime}(z)+r^{\prime}(z)^{2}+r^{\prime \prime}(z) \\
& =: \partial_{z}^{2}+\tilde{p}(z) \partial_{z}+\tilde{q}(z)=\tilde{A}
\end{aligned}
$$

Sandwiching with powers. For $r(z)=\kappa \log (z)$

$$
\begin{aligned}
\left(z-z_{0}\right)^{-\kappa}\left(\partial_{z}^{2}+p(z) \partial_{z}+q(z)\right)\left(z-z_{0}\right)^{\kappa}= & \partial_{z}^{2}+\left(p(z)+2 \kappa\left(z-z_{0}\right)^{-1}\right) \partial_{z}+q(z) \\
& +p(z) \kappa\left(z-z_{0}\right)^{-1}+\left(\kappa^{2}-\kappa\right)\left(z-z_{0}\right)^{-2}
\end{aligned}
$$

If $z_{0} \in \mathbb{C} \cup\{\infty\}$ is a singularity of $(2.2)$ and $\operatorname{rk}\left(z_{0}\right)>1$, then the transformation (2.8) preserves its rank. If $z_{0}$ is a Fuchsian singularity, then after the transformation it is also Fuchsian.

If $\rho_{1}, \rho_{2}$ are the indices of (2.2) at $z_{0} \in \mathbb{C}$, then $\rho_{1}+\kappa, \rho_{2}+\kappa$ are the indices of (2.8) at $z_{0}$. The same is true for $\infty$, except that the indices of (2.8) at $\infty$ are $\rho_{1}-\kappa, \rho_{2}-\kappa$.

Sandwiching with exponentials. Let $k=2,3, \ldots$ We have

$$
\begin{aligned}
\exp ( & \left.\frac{\kappa\left(z-z_{0}\right)^{-k+1}}{k-1}\right)\left(\partial_{z}^{2}+p(z) \partial_{z}+q(z)\right) \exp \left(-\frac{\kappa\left(z-z_{0}\right)^{-k+1}}{k-1}\right) \\
= & \partial_{z}^{2}+\left(p(z)+2 \kappa\left(z-z_{0}\right)^{-k}\right) \partial_{z}+q(z)+p(z) \kappa\left(z-z_{0}\right)^{-k} \\
& +\kappa^{2}\left(z-z_{0}\right)^{-2 k}-\kappa k\left(z-z_{0}\right)^{-k-1} .
\end{aligned}
$$

Hence this transformation preserves $\operatorname{rk}\left(z_{0}\right)$ if it is $>k$, and preserves or decreases $\operatorname{rk}\left(z_{0}\right)$ if it is $=k$. The transformation does not change the coefficients $p_{z_{0},-1}, q_{z_{0},-1}, q_{z_{0},-2}$. Therefore, it also does not change the indices of $z_{0}$.

The same is true for $\infty$ under the transformation

$$
\begin{aligned}
\exp & \left(-\frac{\kappa z^{k+1}}{k+1}\right)\left(\partial_{z}^{2}+p(z) \partial_{z}+q(z)\right) \exp \left(\frac{\kappa z^{k+1}}{k+1}\right) \\
& =\partial_{z}^{2}+\left(p(z)+2 \kappa z^{k}\right) \partial_{z}+q(z)+p(z) \kappa z^{k}+\kappa^{2} z^{2 k}+\kappa k z^{k-1} .
\end{aligned}
$$

More generally, we have transformations of the form (2.7), where

$$
\begin{aligned}
& r(z)=\sum_{k=-m+1}^{-1} \frac{w_{k}}{k}\left(z-z_{0}\right)^{k}+w_{0} \log \left(z-z_{0}\right), \\
& \text { so that } r^{\prime}(z)=\sum_{k=-m+1}^{0} w_{k}\left(z-z_{0}\right)^{k-1} \quad \text { if } \quad z_{0} \in \mathbb{C} ; \\
& r(z)=-\sum_{k=1}^{m-1} \frac{w_{k}}{k} z^{k}-w_{0} \log (z), \\
& \text { so that } r^{\prime}(z)=-\sum_{k=0}^{m-1} w_{k} z^{k-1} \quad \text { if } \quad z_{0}=\infty .
\end{aligned}
$$

We define the absolute rank of $A$ at $z_{0}$ as

$$
\operatorname{Rk}\left(A, z_{0}\right):=\inf \left\{\operatorname{rk}\left(\tilde{A}, z_{0}\right)\right\}
$$

where $\tilde{A}$ are all possible transforms of $A$ of the form (2.7) with $r$ as in (2.11) or (2.12). 


\subsection{Half-integer rank}

In this subsection we discuss singular points with a half-integer rank. They are in a sense exceptional and have special properties.

Suppose that the equation (2.2) has a singular point at $z_{0}$ and $\operatorname{rk}\left(A, z_{0}\right)=m+\frac{1}{2}$, where $m=0,1, \ldots$ It is easy to see that this implies

$$
\operatorname{Rk}\left(A, z_{0}\right)=\operatorname{rk}\left(A, z_{0}\right) .
$$

Without loss of generality we can assume that the singularity is at 0 . This is equivalent to

$$
\begin{aligned}
& \operatorname{deg}(p, 0) \leq m, \quad \operatorname{deg}(q, 0)=2 m+1, \quad m \geq 1, \\
& \operatorname{deg}\left(p-\frac{1}{2 z}, 0\right) \leq 0, \quad \operatorname{deg}(q, 0) \leq 1, \quad m=0 .
\end{aligned}
$$

Let us make the substitution

$$
z=y^{2}, \quad y=\sqrt{z} .
$$

Using $\partial_{z}=\frac{1}{2 y} \partial_{y}$ we transform (2.3) into

$$
\frac{1}{4 y^{2}} \partial_{y}^{2}-\frac{1}{4 y^{3}} \partial_{y}+\frac{p\left(y^{2}\right)}{2 y} \partial_{y}+q\left(y^{2}\right)
$$

Multiplying (2.14) by $4 y^{2}$ we obtain an equation in the principal form

$$
\partial_{y}^{2}+2 y\left(p\left(y^{2}\right)-\frac{1}{2 y^{2}}\right) \partial_{y}+4 y^{2} q\left(y^{2}\right)
$$

Now

$$
\begin{aligned}
& \operatorname{deg}\left(2 y\left(p\left(y^{2}\right)-\frac{1}{2 y^{2}}\right), 0\right) \leq 2 m-1, \\
& \operatorname{deg}\left(4 y^{2} q\left(y^{2}\right), 0\right)=2(2 m+1)-2=4 m, \quad m \geq 1, \\
& \operatorname{deg}\left(4 y^{2} q\left(y^{2}\right), 0\right) \leq 0, \quad m=0 .
\end{aligned}
$$

Thus the rank of (2.15) at zero is $2 m$.

Thus we have shown that by a quadratic substitution we can reduce a singularity of a halfinteger rank to a singularity of integer rank. The resulting equation (2.15) is in addition invariant with respect to the substitution $y \rightarrow-y$ and the rank of the singularity is even.

Note that our definition of rank $\frac{1}{2}$ has been chosen so that the above quadratic reduction works for all half-integer ranks.

\subsection{Simplifying the equation}

Suppose that the equation (2.2) has a singular point at $z_{0}$. Obviously, we have 3 exclusive possibilities:

(0) $\operatorname{Rk}\left(A, z_{0}\right) \leq 1$

(1) $\operatorname{Rk}\left(A, z_{0}\right)$ is an integer and $\geq 2$;

(2) $\operatorname{Rk}\left(A, z_{0}\right)$ is a half-integer and $\geq \frac{3}{2}$.

We would like to simplify the equation around this singularity by sandwiching with $\mathrm{e}^{r}$, where $r$ is given by (2.11) or (2.12). The transformed operator will be, as usual, denoted $\tilde{A}$. We will see that the simplification will be quite different depending on Case (1) and (2). (Case (0) is simple enough, therefore we do not discuss it in the following proposition). 


\section{Proposition 2.1.}

(1) If $\operatorname{Rk}\left(A, z_{0}\right)$ is an integer and $\geq 2$, then there exist exactly two transformations such that

$$
\begin{aligned}
& \operatorname{Rk}\left(A, z_{0}\right)=\operatorname{rk}\left(\tilde{A}, z_{0}\right)=\operatorname{deg}\left(\tilde{p}, z_{0}\right) \geq \operatorname{deg}\left(\tilde{q}, z_{0}\right), \quad z_{0} \in \mathbb{C}, \\
& \operatorname{Rk}(A, \infty)=\operatorname{rk}(\tilde{A}, \infty)=\operatorname{deg}(\tilde{p}, \infty)+2 \geq \operatorname{deg}(\tilde{q}, \infty)+4, \quad z_{0}=\infty
\end{aligned}
$$

(2) If $\operatorname{Rk}\left(A, z_{0}\right)$ is a half-integer and $\geq \frac{3}{2}$, then there exists a unique transformation such that

$$
\begin{aligned}
& \operatorname{deg}\left(\tilde{p}, z_{0}\right) \leq 0 \quad \text { and } \quad \operatorname{Rk}\left(A, z_{0}\right)=\operatorname{rk}\left(\tilde{A}, z_{0}\right)=\frac{1}{2} \operatorname{deg}\left(\tilde{q}, z_{0}\right), \quad z_{0} \in \mathbb{C}, \\
& \operatorname{deg}(\tilde{p}, \infty) \leq-2 \quad \text { and } \quad \operatorname{Rk}(A, \infty)=\operatorname{rk}(\tilde{A}, \infty)=\frac{1}{2} \operatorname{deg}(\tilde{q}, \infty)+2, \quad z_{0}=\infty
\end{aligned}
$$

Proof. Without loss of generality we can assume that the singularity is at 0 . We will use the identity

$$
\tilde{q}(z)=\sum_{n=-2 m}^{\infty} z^{n}\left(\sum_{k=\max (-m, n+1)}^{\min (-1, n+m)} w_{k+1} w_{n-k+1}+(n+1) w_{n+2}+\sum_{k=-m}^{\min (-1, n+m)} w_{k+1} p_{n-k}+q_{n}\right) .
$$

We will apply one of the following three transformations, denoted I, II and III.

Transformation $I$. Suppose that the rank of the initial equation is $m-\frac{1}{2}, m=2,3, \ldots$ Then $\operatorname{deg}(q, 0)=2 m-1$ and

$$
p(z)=\sum_{j=-m+1}^{\infty} p_{j} z^{j} .
$$

We choose $r(z)$ such that

$$
r^{\prime}(z)=-\frac{1}{2} \sum_{j=-m+1}^{-1} p_{j} z^{j}
$$

Then $\operatorname{deg}(\tilde{p}, 0) \leq 0$ and $\operatorname{deg}(\tilde{q}, 0)=\operatorname{deg}(q, 0)$. The transformed equation satisfies $(2.17)$.

For transformations II and III we suppose that the rank of the initial equation is $m=2,3, \ldots$.

Transformation II. Assume that

$$
p_{-m}^{2} \neq 4 q_{-2 m} .
$$

Let $w_{-m+1}$ be one of two solutions of

$$
0=w_{-m+1}^{2}+w_{-m+1} p_{-m}+q_{-2 m} .
$$

Then $\tilde{q}_{-2 m}=0$. Equating $\tilde{q}_{-m+j}=0$ for $j=-m+1, \ldots,-1$ we obtain from (2.18) the recurrence relations

$$
\begin{aligned}
0= & w_{j+1}\left(2 w_{-m+1}+p_{-m}\right) \\
& +\sum_{k=-m+1}^{j-1} w_{k+1} w_{-m+j-k+1}+(-m+j+1) w_{-m+j+2}+\sum_{k=-m}^{j-1} w_{k+1} p_{-m+j-k}+q_{-m+j} .
\end{aligned}
$$

Using

$$
2 w_{-m+1}+p_{-m}=\sqrt{p_{-m}^{2}-4 q_{-2 m}} \neq 0
$$

we can solve the recurrence relations. The transformed equation satisfies (2.16). 
Transformation III. If

$$
p_{-m}^{2}=4 q_{-2 m},
$$

then we sandwich with $\mathrm{e}^{r}$, where $r(z)=\frac{p_{-m} z^{-m+1}}{2(-m+1)}$. The transformed operator $\tilde{A}$ has $\operatorname{deg}(\tilde{p}, 0) \leq$ $m-1$ and $\operatorname{deg}(\tilde{q}, 0) \leq 2 m-1$. Thus after the transformation $\operatorname{rk}(0, \tilde{A}) \leq m-\frac{1}{2}$. If the resulting rank is half-integer, then we apply I and stop. If the resulting rank is an integer, we apply II and stop, or III and we iterate.

We have thus two possibilities:

Case (1) First a finite number of III, and then II. At the end we obtain equation satisfying (2.16). Steps III are uniquely determined and II has two possibilities.

Case (2) First a finite number of III, and then I obtaining (2.17). All steps are uniquely determined.

We will say that the operator $A$ has a grounded form at $z_{0}$ if $\operatorname{deg}\left(p, z_{0}\right) \geq \operatorname{deg}\left(q, z_{0}\right)$. If $z_{0}$ is Fuchsian, then this is equivalent to one of the indices being 0 .

It follows from Proposition 2.1 that if $\operatorname{Rk}\left(A, z_{0}\right)$ is an integer or $\frac{1}{2}$, then the equation can be brought to a grounded form at $z_{0}$.

\subsection{Solutions in terms of formal power series}

We consider the equation (2.2) and try to solve it in terms of a nontrivial, not necessarily convergent power series

$$
\sum_{k=0}^{\infty} v_{k} z^{k}
$$

As we will see, this is not always possible.

Proposition 2.2. Set $m:=\operatorname{deg}(p, 0)$ and $l:=\operatorname{deg}(q, 0)$.

1. If $m \leq 0$ and $l \leq 0$, then for any $v_{0}, v_{1}$ there exists a power series solution.

2. If $m \leq 1$ and $l \leq 2$, then there are no power series solutions unless for some $n=1,2, \ldots$ we have

$$
n(n-1)+n p_{-1}+q_{-2}=0 .
$$

3. If $m \geq 2$ and $l \leq m$, then for any $v_{0}$ there is a unique power series solution.

4. If $m \geq 2$ and $l=m+1$, then there is no power series solution unless for some $n=1,2, \ldots$

$$
p_{-m} n+q_{-m-1}=0 \text {. }
$$

5. If $m \geq 1$ and $l \geq m+2$, then there are no power series solutions.

Proof. By equating the terms at $z^{n}$ to zero in

$$
\left(\partial_{z}^{2}+\sum_{j=-m}^{\infty} p_{j} z^{j} \partial_{z}+\sum_{j=-l}^{\infty} q_{j} z^{j}\right) \sum_{k=0}^{\infty} v_{k} z^{k}=0
$$

we obtain the following equations:

$$
(n+2)(n+1) v_{n+2}+\sum_{k=1}^{n+1+m} p_{n+1-k} k v_{k}+\sum_{k=0}^{n+l} q_{n-k} v_{k}=0, \quad n \in \mathbb{Z} .
$$


Let us prove (5). For $j:=n+l=0, \ldots, l-m-1$ we obtain the equations

$$
\begin{aligned}
& 0=q_{-l} v_{0}, \\
& \text {. } \\
& 0=q_{-l} v_{j}+\cdots+q_{-l+j} v_{0},
\end{aligned}
$$

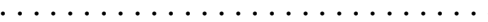

$$
\begin{aligned}
& 0=q_{-l} v_{l-m-1}+\cdots+q_{-m-1} v_{0} .
\end{aligned}
$$

Thus $0=v_{0}=\cdots=v_{l-m-1}$.

If $j \geq l-m$, there are additional terms coming from the 1st order derivative. $v_{k}$ with the highest $k$ contained in such a term has $k=m-l+j+1$. But because of $l \geq m+2$ we have $k \leq j-1$. Thus $v_{k}=0$ by one of previous recursion steps.

If $j \geq l$, there is an additional term coming from the 2nd order derivative, involving $v_{k}$ with $k=-l+j+2$. But $l \geq 3$ implies $k \leq j-1$. Again, this $v_{k}=0$ by one of previous recursion steps.

Let us prove (4). We have recursion relations

$$
\begin{aligned}
& 0=q_{-l} v_{0}, \\
& 0=\left(p_{-l+1}+q_{-l}\right) v_{1}+q_{-l+1} v_{0}, \\
& \ldots \ldots \ldots \ldots \ldots \ldots \ldots \ldots \ldots \ldots \ldots \ldots
\end{aligned}
$$

where dots denote terms depending on $v_{j-1}, \ldots, v_{0}$. If (2.20) has no solutions, then we can solve the recurrence obtaining $0=v_{0}=v_{1}=\cdots$.

Let us prove (3). We have the recursion relations

$$
\begin{aligned}
& 0=p_{-m} v_{1}+q_{-l} v_{0}, \\
& 0=p_{-m} 2 v_{2}+p_{-m+1} v_{1}+q_{-m} v_{1}+q_{-m+1} v_{0} \text {, } \\
& \text {. } \\
& 0=j p_{-m} v_{j}+\cdots,
\end{aligned}
$$

where $\cdots$ involve $v_{0}, \ldots, v_{j-1}$. If $m=2$, there is an additional term $2 v_{0}$ in $(2.21)$. Note that $p_{-m} \neq 0$. Hence, for any $v_{0}$ we can solve the recurrence obtaining $v_{1}, v_{2}, \ldots$

(2) follows immediately from the well-known theory of solutions around a Fuchsian singular point.

(1) is the well-known fact about the Cauchy problem in the regular case.

\subsection{Thomé solutions}

By the so-called Frobenius method, if $z_{0}$ is a Fuchsian singularity and $\rho_{1}, \rho_{2}$ are its indices such that $\rho_{1}-\rho_{2} \notin \mathbb{Z}$, then solutions of the equation (2.2) are spanned by two convergent power series indexed by $i=1,2$ with $v_{i, 0} \neq 0$ :

$$
\begin{aligned}
& \sum_{j=0}^{\infty} v_{i, j}\left(z-z_{0}\right)^{j+\rho_{i}}, \quad z_{0} \in \mathbb{C}, \\
& \sum_{j=-\infty}^{0} v_{i, j} z^{j-\rho_{i}}, \quad z_{0}=\infty .
\end{aligned}
$$


If $\rho_{1}-\rho_{2} \in \mathbb{Z}$ this is not always true. One can then assume that $\rho_{1}-\rho_{2} \geq 0$. There exists one solution as above with $i=1$ and the second has the form

$$
\begin{aligned}
& \sum_{j=0}^{\infty} v_{2, j}\left(z-z_{0}\right)^{\rho_{2}+j}+\log z \sum_{j=0}^{\infty} v_{1, j}\left(z-z_{0}\right)^{\rho_{1}+j}, \quad z_{0} \in \mathbb{C}, \\
& \sum_{j=-\infty}^{0} v_{2, j} z^{-\rho_{2}+j}+\log z \sum_{j=-\infty}^{0} v_{1, j} z^{-\rho_{1}+j}, \quad z_{0}=\infty .
\end{aligned}
$$

If the singular point is not Fuchsian, then we can also look for solutions in a similar form, however the resulting power series are usually no longer convergent. One obtains the so-called Thomé solutions. Note that in some way the situation is simpler, because we do not have the logarithmic case. On the other hand, half-integer ranks need to be treated separately and lead to power series in $\sqrt{z}$.

Proposition 2.3. Let $z_{0}$ be a singular point of $A$ with $\operatorname{rk}\left(A, z_{0}\right)=n$. Then there exist two formal solutions of $A$, indexed by $i=1,2$.

1. If $\operatorname{Rk}\left(A, z_{0}\right)$ is an integer $\geq 2$, they have the form

$$
\begin{aligned}
& \exp \left(\sum_{k=-n+1}^{-1} \frac{\left(z-z_{0}\right)^{k}}{k} w_{i, k}\right) \sum_{j=0}^{\infty} v_{i, j}\left(z-z_{0}\right)^{w_{i, 0}+j}, \quad z_{0} \in \mathbb{C}, \\
& \exp \left(-\sum_{k=1}^{n-1} \frac{z^{k}}{k} w_{i, k}\right) \sum_{j=-\infty}^{0} v_{i, j} z^{-w_{i, 0}+j}, \quad z_{0}=\infty
\end{aligned}
$$

2. Let $\operatorname{Rk}\left(A, z_{0}\right)$ be a half integer $\geq \frac{3}{2}$. The two formal solutions have the form

$$
\begin{aligned}
& \exp \left(\sum_{k=-n+1}^{-\frac{1}{2}} \frac{\left(z-z_{0}\right)^{k}}{k} w_{i, k}\right) \sum_{j=0}^{\infty} v_{i, j}\left(z-z_{0}\right)^{w_{i, 0}+j}, \quad z_{0} \in \mathbb{C}, \\
& \exp \left(-\sum_{k=\frac{1}{2}}^{n-1} \frac{z^{k}}{k} w_{i, k}\right) \sum_{j=-\infty}^{0} v_{i, j} z^{-w_{i, 0}+j}, \quad z_{0}=\infty .
\end{aligned}
$$

Here $\sum^{\prime}$ denotes the sum where the index $k$ within its range runs over both integers and half-integers. We have

$$
w_{0, k}=(-1)^{2 k} w_{1, k}, \quad v_{0, k}=(-1)^{2 k} v_{1, k} .
$$

Proof. For simplicity, assume that $z_{0}=0$.

Suppose that $\operatorname{Rk}(A, 0)$ is an integer $\geq 2$. By Proposition 2.1(1) we can transform the equation to a grounded form in two distinct ways. By Proposition 2.2(3), the grounded form has a solution in terms of the power series.

If $\operatorname{Rk}(A, 0)=m+\frac{1}{2}$ is a half-integer $\geq \frac{3}{2}$, first we reduce the equation to the form with a half-integer rank, see Proposition 2.1(2). Then we apply the quadratic transformation, as described in Section 2.5. We obtain an even equation in $\sqrt{z}$ of the rank $2 m$, with $\tilde{p}_{-2 m}=0$ and $\tilde{q}_{-4 m} \neq 0$. We already know that it has a solution of the form described in 1 :

$$
\exp \left(\sum_{k=-2 m+1}^{-1} \frac{(\sqrt{z})^{k}}{k} \tilde{w}_{i, k}\right) \sum_{j=0}^{\infty} \tilde{v}_{j}(\sqrt{z})^{\tilde{w}_{i, 0}+j} .
$$


Using $2 m \geq 2$ and (2.19) we obtain

$$
\tilde{w}_{-2 m+1}=\sqrt{-\tilde{q}_{-4 m}} \neq 0 .
$$

Clearly, (2.23) with $\sqrt{z}$ replaced by $-\sqrt{z}$ is also a solution. By $(2.24)$ both solutions are not proportional to one another. This proves (2.3).

Note that Proposition 2.3 is also true in the Fuchsian case, except that for $\operatorname{Rk}\left(A, z_{0}\right)=1$, one has to make an obvious modification in the logarithmic case, and for $\operatorname{Rk}\left(A, z_{0}\right)=\frac{1}{2}(2.3)$ does not have to be true.

In the Fuchsian case $w_{i, 0}, i=1,2$, coincide with the indices of $z_{0}$. In what follows, the numbers $w_{i, 0}, i=1,2$, will be called indices of $z_{0}$ in the general case as well. We also introduce the alternative notation

$$
\rho_{z_{0}, i}:=w_{i, 0}, \quad i=1,2 .
$$

Proposition 2.4. Let $z_{0}$ be a singularity of $A$.

1. We have

$$
\begin{aligned}
& \rho_{z_{0}, 1}+\rho_{z_{0}, 2}=-p_{z_{0},-1}+\operatorname{Rk}\left(A, z_{0}\right), \quad z_{0} \in \mathbb{C}, \\
& \rho_{\infty, 1}+\rho_{\infty, 2}=p_{\infty,-1}-2+\operatorname{Rk}(A, \infty), \quad z_{0}=\infty .
\end{aligned}
$$

2. If $\operatorname{Rk}\left(A, z_{0}\right) \in\left\{\frac{3}{2}, \frac{5}{2}, \ldots\right\}$, then

$$
\begin{aligned}
& \rho_{z_{0}, 1}=\rho_{z_{0}, 2}=\frac{1}{2}\left(-p_{z_{0},-1}+\operatorname{Rk}\left(A, z_{0}\right)\right), \quad z_{0} \in \mathbb{C}, \\
& \rho_{\infty, 1}=\rho_{\infty, 2}=\frac{1}{2}\left(p_{\infty,-1}-2+\operatorname{Rk}(A, \infty)\right), \quad z_{0}=\infty .
\end{aligned}
$$

3. If $z_{0} \in \mathbb{C}$ is grounded, then

$$
\left\{\rho_{z_{0}, 1}, \rho_{z_{0}, 2}\right\}=\left\{0,-p_{z_{0},-1}+\operatorname{Rk}\left(A, z_{0}\right)\right\} .
$$

Proof. (1) Without loss of generality we can assume that $z_{0}=0$. When we apply sandwiching with $\mathrm{e}^{r}$, where $r(z)$ has the form (2.11), then $p_{-1}$ and $\rho_{i}$ are transformed into $p_{-1}-2 w_{0}$ and $\rho_{i}+w_{0}$. This does not affect the identity (2.26).

Assume first that $\operatorname{Rk}(A, 0)$ is an integer. Then by a sandwiching transformation we can reduce the equation to a grounded form at 0 . The first $m$ recurrence relations of (2.18) read then

$$
\begin{aligned}
& 0=\sum_{k=-m}^{n+m} w_{k+1}\left(w_{n-k+1}+p_{n-k}\right), \quad n=-2 m, \ldots,-m-2, \\
& 0=\sum_{k=-m}^{-1} w_{k+1}\left(w_{-m-k}+p_{-m-1-k}\right)-m w_{-m+1} .
\end{aligned}
$$

Apart from the solution $w_{k+1}=0, k=-m, \ldots,-1$, this is solved by

$$
\begin{aligned}
& w_{k+1}:=-p_{k}, \quad k=-m, \ldots,-2, \\
& w_{0}:=-p_{-1}+m .
\end{aligned}
$$

Thus $\left\{\rho_{1}, \rho_{2}\right\}=\left\{0,-p_{-1}+m\right\}$. Hence $(2.26)$ is satisfied. 
Assume next that $\operatorname{Rk}(A, 0)$ is a half integer equal $m+\frac{1}{2}$. After an appropriate sandwiching transformation we can assume that $\operatorname{Rk}(A, 0)=\operatorname{rk}(A, 0)$. Then we can apply the quadratic transformation (2.13) obtaining an equation

$$
\tilde{A}=\partial_{y}^{2}+\tilde{p}(y) \partial_{y}+\tilde{q}(y) .
$$

Let $\tilde{\rho}_{i}, i=1,2$ be the indices of $\tilde{A}$ at zero. Now $\operatorname{rk}(\tilde{A}, 0)=2 m$, which is an integer. Hence we can apply the formula (2.26)

$$
\tilde{\rho}_{1}+\tilde{\rho}_{2}=-\tilde{p}_{-1}+2 m \text {. }
$$

But the indices of $A$ at 0 are $\rho_{i}=\frac{\tilde{\rho}_{i}}{2}, i=1,2$ and $\tilde{p}_{-1}=2 p_{-1}-1$. Hence

$$
\rho_{1}+\rho_{2}=\frac{1}{2}\left(\tilde{\rho}_{1}+\tilde{\rho}_{2}\right)=\frac{1}{2}\left(-2 p_{-1}+1+2 m\right)=-p_{-1}+m+\frac{1}{2} .
$$

This ends the proof (2.26).

To prove (2.27) we apply the transformation $w=\frac{1}{z}$. We note that $\operatorname{Rk}(A, \infty)$ is transformed to $\operatorname{Rk}(A, 0)$ and $\rho_{\infty, i}$ are transformed to $\rho_{0, i}, i=1,2$. Besides, $p_{\infty,-1}$ is transformed to $-p_{0,-1}+2$.

(1) and (2.3) imply (2).

(1) and (2.2) of Proposition 2.2 imply (3).

\subsection{Nonlogarithmic singularities}

Let $z_{0}$ be a singular point of the equation (2.2). We say that $z_{0}$ is nonlogarithmic or apparent iff all solutions of the equation are meromorphic around this singularity. If $z_{0}$ is a nonlogarithmic Fuchsian singular point, then both its indices are integers.

The following proposition shows how to deform a given equation so that one obtains an additional nonlogarithmic singularity with indices 0,2 . This deformation depends on two parameters $\lambda, \mu$ : the additional singularity is located at $\lambda$, and solutions of the deformed equation satisfy $\mu v(\lambda)=v^{\prime}(\lambda)$. It will play the central role in the derivation of Painlevé equations from Heun class equations in Section 4.

Proposition 2.5. Let $\sigma, \tau, \eta$ be analytic at $\lambda$ and $\sigma(\lambda) \neq 0$. Let $\mu \in \mathbb{C}$. Then all solution of the equation given by

$$
\sigma(z) \partial_{z}^{2}+\left(\tau(z)-\frac{\sigma(z)}{z-\lambda}\right) \partial_{z}+\eta(z)-\eta(\lambda)-\mu^{2} \sigma(\lambda)-\mu\left(\tau(\lambda)-\sigma^{\prime}(\lambda)\right)+\frac{\mu \sigma(\lambda)}{z-\lambda}
$$

are analytic at $\lambda$. Thus the equation given by (2.28) has a nonlogarithmic singularity at $z=\lambda$. The singularity is Fuchsian with indices $0,2$.

Proof. We look for a solution analytic around $\lambda$ :

$$
v(z)=\sum_{n=0}^{\infty} v_{n}(z-\lambda)^{n} .
$$

We obtain

$$
\begin{aligned}
& \left(-\sigma(\lambda) v_{1}+\mu \sigma(\lambda) v_{0}\right)(z-\lambda)^{0}+\left(\sigma(\lambda) 2 v_{2}+\left(\tau(\lambda)-\sigma^{\prime}(\lambda)\right) v_{1}-\sigma(\lambda) 2 v_{2}-(\mu \tau(\lambda)\right. \\
& \left.\left.\quad-\mu \sigma^{\prime}(\lambda)+\mu^{2} \sigma(\lambda)\right) v_{0}+\mu \sigma(\lambda) v_{1}\right)(z-\lambda)^{1} \\
& \quad+\sum_{n=3}^{\infty}\left(\sigma(\lambda) n(n-2) v_{n}+\cdots\right)(z-\lambda)^{n-1}=0
\end{aligned}
$$

We have $\sigma(\lambda) \neq 0$. Hence the first line implies $v_{1}=\mu v_{0}$. Then the second line is identically zero, and $v_{2}$ is left unspecified. The next terms yield recurrence relations for $v_{n}, n=3, \ldots$. 


\section{Equations with rational coefficients}

\subsection{The $M_{n}$ class and the grounded $M_{n}$ class}

Consider an equation given by the operator

$$
A:=\partial_{z}^{2}+p(z) \partial_{z}+q(z)
$$

where $p(z), q(z)$ are rational functions.

If $z_{1}, \ldots, z_{k} \in \mathbb{C} \cup\{\infty\}$ are its singularities and their ranks are $m_{1}, \ldots, m_{k}$, then we will say that the equation (3.1) is of type $\left(m_{1} m_{2} \cdots m_{k}\right)$

Often we will need a more precise description of (3.1), which gives information what is the rank of the singularity at $\infty$. We will then put it at the end of the sequence, so that $z_{k}=\infty$, and precede it with a semicolon. We will write that (3.1) is of type $\left(m_{1} m_{2} \cdots m_{k-1} ; m_{\infty}\right)$.

By writing $m_{i}$ instead of $m_{i}$ we will mean the rounded rank. We will use it especially often for 1 . Thus $\underline{1}$ means a Fuchsian singularity $\left(1\right.$ or $\left.\frac{1}{2}\right)$.

Every equation having no more than $n+1$ singular points in the Riemann sphere, all of them Fuchsian and at most $n$ finite, is given by an operator of the form

$$
\partial_{z}^{2}+\sum_{j=1}^{n} \frac{a_{j}}{z-z_{j}} \partial_{z}+\sum_{j=1}^{n} \frac{b_{j}}{z-z_{j}}+\sum_{j=1}^{n} \frac{c_{j}}{\left(z-z_{j}\right)^{2}} \quad \text { with } \quad \sum_{j=1}^{n} b_{j}=0
$$

where $z_{1}, \ldots, z_{n}$ are distinct points in $\mathbb{C}$. The family of equations $(3.2)$ will be called the $M_{n}$ type. The corresponding symbol is $(\underset{n-1}{\underline{1} \text { times }} ; \underline{1})$.

Each finite singularity has at least one index equal 0 if and only if $c_{1}=\cdots=c_{n-1}=0$. Thus such equations are given by operators

$$
\partial_{z}^{2}+\sum_{j=1}^{n} \frac{a_{j}}{z-z_{j}} \partial_{z}+\sum_{j=1}^{n} \frac{b_{j}}{z-z_{j}} \quad \text { with } \quad \sum_{j=1}^{n} b_{j}=0 .
$$

The family of equations given by (3.3) will be called the grounded $M_{n}$ type.

Proposition 3.1. By sandwiching with powers, as in (2.8), we can always transform an $M_{n}$ type equation into a grounded $M_{n}$ type equation.

We say that a differential equation belongs to the $M_{n}$ class if it is given by

$$
\partial_{z}^{2}+\frac{\tau(z)}{\sigma(z)} \partial_{z}+\frac{\xi(z)}{\sigma(z)^{2}}
$$

where $\sigma, \tau, \xi$ are polynomials satisfying

$$
\sigma \neq 0, \quad \operatorname{deg} \sigma \leq n, \quad \operatorname{deg} \tau \leq n-1, \quad \operatorname{deg} \xi \leq 2 n-2 .
$$

We will often use the shorthand

$$
\eta(z):=\frac{\xi(z)}{\sigma(z)}
$$

where $\eta$ does not have to be a polynomial.

We say that a differential equation belongs to the grounded $M_{n}$ class if it is given by

$$
\partial_{z}^{2}+\frac{\tau(z)}{\sigma(z)} \partial_{z}+\frac{\eta(z)}{\sigma(z)},
$$

where $\sigma, \tau, \eta$ are polynomials satisfying

$$
\sigma \neq 0, \quad \operatorname{deg} \sigma \leq n, \quad \operatorname{deg} \tau \leq n-1, \quad \operatorname{deg} \eta \leq n-2 .
$$

The name "the $M_{n}$ class" is borrowed from Lay-Slavyanov [25]. 


\section{Proposition 3.2.}

1. The $M_{n}$ type is contained in the $M_{n}$ class. An equation of the $M_{n}$ class is of the $M_{n}$ type iff $\sigma$ possesses $n$ distinct roots.

2. The grounded $M_{n}$ type is contained in the grounded $M_{n}$ class. An equation of the grounded $M_{n}$ class is of the grounded $M_{n}$ type iff $\sigma$ possesses $n$ distinct roots.

Proof. Let us prove (1). Consider (3.2). Set

$$
\sigma(z):=\left(z-z_{1}\right) \cdots\left(z-z_{n}\right) .
$$

Then clearly $\sigma$ is a nonzero polynomial with $n$ distinct roots. We easily see that (3.2) can be rewritten as (3.4) with (3.5) satisfied.

Conversely, consider (3.4) such that $\sigma$ has $n$ distinct roots, namely, $z_{1}, \ldots, z_{n}$. Then we can decompose $\frac{\tau(z)}{\sigma(z)}$ and $\frac{\xi(z)}{\sigma(z)^{2}}$ into simple fractions, obtaining (3.2).

The proof of (2) is analogous.

Proposition 3.3. Let $\left(z_{1}, \ldots, z_{k}\right)$ be the singularities of an equation $A$ of the $M_{n}$ class. Then

$$
\lceil\operatorname{rk}\rceil\left(A, z_{1}\right)+\cdots+\lceil\mathrm{rk}\rceil\left(A, z_{k}\right) \leq n+1 .
$$

Proof. Without loss of generality we can assume that $z_{1}, \ldots, z_{k-1} \in \mathbb{C}$ and $z_{k}=\infty$. Let

$$
\sigma(z)=\left(z-z_{1}\right)^{m_{1}} \cdots\left(z-z_{k-1}\right)^{m_{k-1}}
$$

with distinct $z_{i}$ 's. Then $z_{1}, \ldots, z_{k-1}$ are the finite singular points. Clearly, $\lceil\operatorname{rk}\rceil\left(A, z_{i}\right) \leq m_{i}$. Therefore,

$$
\lceil\mathrm{rk}\rceil\left(A, z_{1}\right)+\cdots+\lceil\mathrm{rk}\rceil\left(A, z_{k-1}\right) \leq \operatorname{deg} \sigma .
$$

Now

$$
\begin{aligned}
\lceil\mathrm{rk}\rceil(A, \infty) & =\left\lceil\max \left(\operatorname{deg} \tau-\operatorname{deg} \sigma+2, \frac{1}{2}(\operatorname{deg} \xi-2 \operatorname{deg} \sigma)+2\right)\right] \\
& \leq\left\lceil\max \left(n-1-\operatorname{deg} \sigma+2, \frac{1}{2}(2 n-2-2 \operatorname{deg} \sigma)+2\right)\right]=n+1-\operatorname{deg} \sigma .
\end{aligned}
$$

Then we sum (3.7) and (3.8).

We will often represent $M_{n}$ class equations by operators obtained by multiplying (3.4) or (3.6) from the right by $\sigma(z)$ :

$$
\sigma(z) \partial_{z}^{2}+\tau(z) \partial_{z}+\eta(z) .
$$

Obviously, $M_{n}$ class equations and operators are defined by coefficients of the polynomials $\sigma$, $\tau, \xi$. Therefore, they form a complex manifold parameterized by

$$
\left(\mathbb{C}^{n} \backslash\{0\}\right) \times \mathbb{C}^{3 n-3} .
$$

The condition saying that $\sigma$ has $n$ distinct roots defines an open dense subset in (3.10). Thus the $M_{n}$ type is an open dense subset of the $M_{n}$ class. Hence the $M_{n}$ class consists of the $M_{n}$ type and its limiting points in the topology of (3.10). These limiting points are traditionally called confluent cases.

Similarly, grounded $M_{n}$ class equations and operators are defined by $\sigma, \tau, \eta$. Therefore, they form a complex manifold parameterized by

$$
\left(\mathbb{C}^{n} \backslash\{0\}\right) \times \mathbb{C}^{2 n-3}
$$

Clearly, the grounded $M_{n}$ type is an open dense subset of the grounded $M_{n}$ class. One can say that the grounded $M_{n}$ class consists of the grounded $M_{n}$ type and its confluent cases. 


\subsection{Generalized Fuchs relation}

Recall that for any singular point $z_{0}$ of an equation $A$ we defined its two indices $\rho_{z_{0}, 1}$ and $\rho_{z_{0}, 2}$. For Fuchsian singularities they were defined in (2.4), (2.5) and for non-Fuchsian singularities in (2.25). If all singularities are regular then the well-known Fuchs relation says that the sum of all indices equals the number of singularities minus 2 . In the following proposition we describe its generalization which is valid if some of the singularities are non-Fuchsian.

Proposition 3.4. Let $z_{1}, \ldots, z_{k}$ be the singular points of an equation A. Then

$$
\sum_{j=1}^{k}\left(\rho_{z_{j}, 1}+\rho_{z_{j}, 2}\right)=\sum_{j=1}^{k} \operatorname{Rk}\left(A, z_{j}\right)-2 .
$$

Proof. Without loss of generality we can assume that $z_{k}=\infty$. We have

$$
\begin{aligned}
& \rho_{z_{i}, 1}+\rho_{z_{i}, 2}=-p_{z_{j},-1}+\operatorname{Rk}\left(A, z_{j}\right), \quad j=1, \ldots, k-1, \\
& \rho_{\infty, 1}+\rho_{\infty, 2}=p_{\infty,-1}-2+\operatorname{Rk}(A, \infty) \\
& p_{\infty,-1}=\sum_{j=1}^{k-1} p_{z_{j},-1}
\end{aligned}
$$

where (3.12) and (3.13) follows from Proposition 2.4. Summing up the above three relations we obtain (3.11).

\subsection{Riemann class equations}

The simplest nontrivial $M_{n}$ class is the $M_{2}$ class. We call it the Riemann class since it consists of the Riemann equation with one singularity at $\infty$ and its confluent cases. Thus Riemann class operators have the form (3.4), where

$$
\sigma \neq 0, \quad \operatorname{deg} \sigma \leq 2, \quad \operatorname{deg} \tau \leq 1, \quad \operatorname{deg} \xi \leq 2 .
$$

The grounded Riemann class operators has the form (3.6), where

$$
\sigma \neq 0, \quad \operatorname{deg} \sigma \leq 2, \quad \operatorname{deg} \tau \leq 1, \quad \eta \quad \text { is a number. }
$$

Note that grounded Riemann class equations appear in the literature very often. They are often called hypergeometric type equations, see [2, 4, 18].

It is well known that by a division by a constant, transformations $z \mapsto a z+b$, sandwiching with powers and exponentials all Riemann class operators can be transformed into one of the following types:

\begin{tabular}{lcl}
\hline the ${ }_{2} F_{1}$ operator & $(\underline{11} ; \underline{1})$ & $z(1-z) \partial_{z}^{2}+(c-(a+b+1) z) \partial_{z}-a b$ \\
\hline the ${ }_{2} F_{0}$ operator & $(2 ; \underline{1})$ & $z^{2} \partial_{z}^{2}+(-1+(a+b+1) z) \partial_{z}+a b$ \\
\hline the ${ }_{1} F_{1}$ operator & $(\underline{1} ; 2)$ & $z \partial_{z}^{2}+(c-z) \partial_{z}-a$ \\
\hline the ${ }_{0} F_{1}$ operator & $\left(\underline{1} ; \frac{3}{2}\right)$ & $z \partial_{z}^{2}+c \partial_{z}-1$ \\
\hline the Hermite operator & $(; 3)$ & $\partial_{z}^{2}-2 z \partial_{z}-2 a$ \\
\hline the Airy operator & $\left(; \frac{5}{2}\right)$ & $\partial_{z}^{2}+z$ \\
\hline the Euler II operator & $(\underline{1} ; \underline{1})$ & $z^{2} \partial_{z}^{2}+c z \partial_{z}$ \\
\hline the Euler I operator & $(\underline{1} ; \underline{1})$ & $z \partial_{z}^{2}+c \partial_{z}$ \\
\hline the 1d Helmholtz operator & $(; 2)$ & $\partial_{z}^{2}+1$ \\
\hline the 1d Laplace operator & $(; \underline{1})$ & $\partial_{z}^{2}$ \\
\hline
\end{tabular}


Let us make some remarks.

1. The Euler II and Euler I operators yield the same equations.

2. The last four equations from the table can be solved in elementary functions.

3. In this table, only the Airy equation cannot be brought to the grounded form.

4. When we take into account the transformation $z \mapsto z^{-1}$, then the types $(2 ; \underline{1})$ and $(\underline{1} ; 2)$ are equivalent.

5. There are more relations between various types when we consider more complicated transformations.

\subsection{Heun class equations}

$M_{3}$ type equations were studied by Heun in [8]. Therefore, it is natural to call the $M_{3}$ type the Heun type. Consequently, the $M_{3}$ class will be called the Heun class. The grounded $M_{3}$ class will be called the grounded Heun class.

Our terminology is consistent with [24, 25]. However, in some publications the name Heun class is used to denote what we call the grounded Heun class, see, e.g., [5].

We will represent Heun class equations by Heun class operators. More precisely, we will say that

$$
\sigma(z) \partial_{z}^{2}+\tau(z) \partial_{z}+\eta(z)
$$

is a Heun class operator if $\eta(z)=\frac{\xi(z)}{\sigma(z)}$ and $\sigma, \tau, \xi$ are polynomials such that

$$
\sigma \neq 0, \quad \operatorname{deg} \sigma \leq 3, \quad \operatorname{deg} \tau \leq 2, \quad \operatorname{deg} \xi \leq 4 .
$$

(3.14) is a grounded Heun class operator if $\sigma, \tau, \eta$ are polynomials such that

$\sigma \neq 0, \quad \operatorname{deg} \sigma \leq 3, \quad \operatorname{deg} \tau \leq 2, \quad \operatorname{deg} \eta \leq 1$.

If in addition $\sigma$ has 3 distinct roots, then (3.14) is a (grounded) Heun type operator.

Clearly, the Heun class and the grounded Heun class are preserved by transformations $z \mapsto$ $a z+b$.

The Heun class is also preserved by sandwiching with powers and exponentials, see (2.8), (2.9) and (2.10).

Heun class operators are invariant with respect to swapping a finite singularity with the infinity. More precisely, Heun class operators of the form (3.14) after the transformations

$$
w=\left(z-z_{0}\right)^{-1} \text {, where } z_{0} \text { is one of finite singular points }
$$

remain in the Heun class. Indeed, without loss of generality, we can suppose that $z_{0}=0$. Thus $\sigma(0)=0$, so that $\sigma(z)=z \rho(z)$, where $\rho$ is a polynomial with $\operatorname{deg} \rho \leq 2$. Substitute $w=z^{-1}$, which transforms (3.14) into

$$
w^{3} \rho\left(w^{-1}\right) \partial_{w}^{2}+w^{2}\left(2 \rho\left(w^{-1}\right)-\tau\left(w^{-1}\right)\right) \partial_{w}+\eta\left(w^{-1}\right) .
$$

It is easy to see that

$$
\begin{aligned}
& \tilde{\sigma}(w):=w^{3} \rho\left(w^{-1}\right), \\
& \tilde{\tau}(w):=w^{2}\left(2 \rho\left(w^{-1}\right)-\tau\left(w^{-1}\right)\right), \\
& \tilde{\eta}(w):=\eta\left(w^{-1}\right)
\end{aligned}
$$

still satisfy the condition (3.15). 
Note that we do not need to put any prefactor in (3.16). Remarkably, the analogous property does not hold for the $M_{n}$ classes with $n \neq 3$ : for them after swapping a finite singularity with $\infty$ an additional prefactor is needed.

Swapping a finite singularity with $\infty$ is possible also if we want to stay within the grounded Heun class, except that the transformation $w=\left(z-z_{0}\right)^{-1}$ needs to be followed by sandwiching with a power, that is a transformation (2.8). Indeed, assume (3.9) is a grounded Heun class operator and $z_{0}=0$ is a singularity. Let $\alpha$ satisfy the generalized indicial equation at $z=\infty$ :

$$
\frac{\rho^{\prime \prime}}{2} \alpha(\alpha+1)-\frac{\tau^{\prime \prime}}{2} \alpha+\eta^{\prime}=0
$$

Then

$$
w^{-\alpha}\left(\tilde{\sigma}(w) \partial_{w}^{2}+\tilde{\tau}(w) \partial_{w}+\tilde{\eta}(w)\right) w^{\alpha}=\tilde{\sigma}(w) \partial_{w}^{2}+\tilde{\tau}_{1}(w) \partial_{w}+\tilde{\eta}_{1}(w),
$$

where

$$
\begin{aligned}
& \tilde{\tau}_{1}(w):=2(\alpha+1) w^{2} \rho\left(w^{-1}\right)-w^{2} \tau\left(w^{-1}\right), \\
& \tilde{\eta}_{1}(w):=w \alpha((\alpha+1) \rho(0)-\tau(0))+\alpha\left((\alpha+1) \rho^{\prime}(0)-\tau^{\prime}(0)\right)+\eta(0) .
\end{aligned}
$$

Clearly, (3.18) is a grounded Heun class operator.

\subsection{Deformed Heun class equations}

Consider $\sigma, \tau, \eta$ satisfying the conditions (3.15), so that (3.14) is a Heun class operator. Let $\lambda, \mu \in \mathbb{C}$. The corresponding deformed Heun class operator is defined as

$$
\sigma(z) \partial_{z}^{2}+\left(\tau(z)-\frac{\sigma(z)}{z-\lambda}\right) \partial_{z}+\eta(z)-\eta(\lambda)-\sigma(\lambda) \mu^{2}-\left(\tau(\lambda)-\sigma^{\prime}(\lambda)\right) \mu+\frac{\sigma(\lambda) \mu}{z-\lambda} .
$$

By Proposition 2.5, the equation defined by (3.19) has a nonlogarithmic singularity at $z=\lambda$ with indices 0,2 . All the remaining finite singularities have the same type (the rank, the indices), as for the original Heun class operator (3.14).

Thus to every Heun class operator (3.14) there corresponds a family of deformed Heun class operators (3.19) depending on two new parameters: $\lambda$ and $\mu$. Note that one of the parameters of $\eta$ in the original operator (2.28) is lost - (3.19) does not depend on the free (zeroth order) term of $\eta$.

The family of deformed Heun class operators is preserved by the same transformations as the family of Heun class operators. Clearly, it is preserved by $z \mapsto a z+b$, division by a constant and sandwiching with powers and exponentials, as described in (2.8), (2.9) and (2.10).

It is also invariant with respect to swapping the singularity at $\infty$ with finite singularities. Thus assume that $\sigma(z)=z \rho(z)$. Then substitution $w=z^{-1}$ transforms (3.19) into

$$
\begin{aligned}
\tilde{\sigma}(w) \partial_{w}^{2} & +\left(\tilde{\tau}(w)-\frac{\tilde{\sigma}(w)}{w-\lambda^{-1}}\right) \partial_{w}+\tilde{\eta}(w)-\tilde{\eta}\left(\lambda^{-1}\right)-\tilde{\sigma}\left(\lambda^{-1}\right) \mu^{2} \\
& -\left(\tilde{\tau}\left(\lambda^{-1}\right) \tilde{\sigma}^{\prime}\left(\lambda^{-1}\right)\right) \mu+\frac{\tilde{\sigma}\left(\lambda^{-1}\right) \mu}{w-\lambda^{-1}}
\end{aligned}
$$

where

$$
\begin{aligned}
& \tilde{\sigma}(w):=w^{3} \rho\left(w^{-1}\right), \\
& \tilde{\tau}(w):=w^{2}\left(3 \rho\left(w^{-1}\right)-\tau\left(w^{-1}\right)\right), \\
& \tilde{\eta}(w):=\eta\left(w^{-1}\right), \\
& \tilde{\mu}:=-\lambda^{2} \mu .
\end{aligned}
$$


Transformation $w=z^{-1}$ transforms a deformed Heun class operator satisfying $\sigma(0)=0$ into another deformed Heun class operator satisfying $\tilde{\sigma}(0)=0$, similarly as for undeformed Heun class operators. Note however a subtle difference between (3.17b) and (3.20b).

The following proposition describes mapping properties of the above described transformation in more detail:

\section{Proposition 3.5.}

1. $\sigma \eta(0)=0 \Leftrightarrow \operatorname{deg} \tilde{\sigma} \tilde{\eta} \leq 3$.

2. $\sigma^{\prime}(0)=0 \Leftrightarrow \operatorname{deg} \tilde{\sigma} \leq 2$.

3. $\sigma^{\prime}(0)=0$ and $\tau(0)=0 \Leftrightarrow \operatorname{deg} \tilde{\sigma} \leq 2$ and $\operatorname{deg} \tilde{\tau} \leq 1$.

4. $\sigma^{\prime}(0)=0$ and $\left(\sigma \eta(0)=(\sigma \eta)^{\prime}(0)=0 \Leftrightarrow \operatorname{deg} \tilde{\sigma} \leq 2\right.$ and $\operatorname{deg} \tilde{\sigma} \tilde{\eta} \leq 2$.

\subsection{Classification of Heun class equations}

In this subsection we discuss two classifications of Heun class equations and operators.

The first is based on the rank of singularities. We classify half-integer and integer ranks separately, except for the rank 1, where we use, as usual, the rounded rank. Not counting the types reducible to the Riemann class, which are treated as "trivial", it partitions the Heun class into ten types.

There exists also a coarser classification, which uses rounded singularity ranks. It groups the ten nontrivial types of the Heun class into five supertypes.

In the following list we give both classifications of the Heun class:

- (standard) Heun or (1111).

- confluent Heun or $(\underline{112})$.

- non-degenerate confluent Heun or $(\underline{112})$.

- degenerate confluent Heun or $\left(\underline{11} \frac{3}{2}\right)$.

- doubly confluent Heun or $(\underline{22})$.

- non-degenerate doubly confluent Heun or (22).

- degenerate doubly confluent Heun or $\left(\frac{3}{2} 2\right)$.

- doubly degenerate doubly confluent Heun or $\left(\frac{3}{2} \frac{3}{2}\right)$.

- biconfluent Heun or $(\underline{13})$.

- non-degenerate biconfluent Heun or (13).

- degenerate biconfluent Heun $\left(\underline{1} \frac{5}{2}\right)$.

- triconfluent Heun ( $\underline{4})$.

- non-degenerate triconfluent Heun (4).

- degenerate triconfluent Heun $\left(\frac{7}{2}\right)$.

In the above list we use names similar to those proposed by [25].

Some of the types in this list have two distinct varieties, which are equivalent by swapping a finite singularity with infinity. The variety where the higher rank singularity is put at $\infty$ is sometimes called the natural. For instance, $(\underline{112})$ has the natural variety $(\underline{11} ; \underline{2})$ and the alternative variety $(\underline{21} ; \underline{1})$.

For some varieties we give more than one normal form - they are labelled a) and b).

In the following theorem we describe normal forms of various types of Heun class operators. Note that there is some arbitrariness in the choice of a normal form. We allow the following transformations: $z \mapsto a z+b$, division by a constant, sandwiching with powers and exponentials. 
Theorem 3.6. Each Heun class operator can be transformed into a Riemann class operator or one of the following normal forms:

\begin{tabular}{|c|c|c|c|c|}
\hline type & $\sigma(z)$ & $\tau(z)$ & $\eta(z)$ & \\
\hline$(\underline{111} ; \underline{1})$ & $z(z-1)(z-t)$ & $a_{2} z^{2}+a_{1} z+a_{0}$ & $\begin{array}{l}\text { a) } b_{1} z+b_{0} \\
\text { b) } b_{0}+b_{-1} z^{-1}\end{array}$ & $t \neq 0,1$ \\
\hline$(\underline{11} ; 2)$ & $z(z-1)$ & $a_{2} z^{2}+a_{1} z+a_{0}$ & $\begin{array}{l}\text { a) } b_{1} z+b_{0} \\
\text { b) } b_{0}+b_{-1} z^{-1}\end{array}$ & $a_{2} \neq 0$ \\
\hline$(2 \underline{1} ; \underline{1})$ & $z^{2}(z-1)$ & $a_{2} z^{2}+a_{1} z+a_{0}$ & $\begin{array}{l}\text { a) } b_{1} z+b_{0} \\
\text { b) } b_{0}+b_{-1} z^{-1}\end{array}$ & $a_{0} \neq 0$ \\
\hline$\left(\underline{11} ; \frac{3}{2}\right)$ & $z(z-1)$ & $a_{1} z+a_{0}$ & $b_{1} z+b_{0}$ & $b_{1} \neq 0$ \\
\hline$\left(\frac{3}{2} \underline{1} ; \underline{1}\right)$ & $z^{2}(z-1)$ & $a_{2} z^{2}+a_{1} z$ & $b_{0}+b_{-1} z^{-1}$ & $b_{-1} \neq 0$ \\
\hline$(2 ; 2)$ & $z^{2}$ & $a_{2} z^{2}+a_{1} z+a_{0}$ & $\begin{array}{l}\text { a) } b_{1} z+b_{0} \\
\text { b) } b_{0}+b_{-1} z^{-1}\end{array}$ & $a_{2} \neq 0, a_{0}=c$ \\
\hline$\left(\frac{3}{2} ; 2\right)$ & $z^{2}$ & $a_{2} z^{2}+a_{1} z$ & $b_{0}+b_{-1} z^{-1}$ & $b_{-1} \neq 0, a_{2}=c$ \\
\hline$\left(2 ; \frac{3}{2}\right)$ & $z^{2}$ & $a_{1} z+a_{0}$ & $b_{1} z+b_{0}$ & $b_{1} \neq 0, a_{0}=c$ \\
\hline$\left(\frac{3}{2} ; \frac{3}{2}\right)$ & $z^{2}$ & 0 & $b_{1} z+b_{0}+b_{-1} z^{-1}$ & $b_{-1} \neq 0, b_{1}=c$ \\
\hline$\left(1 ; \frac{3}{2}\right)$ & $z^{2}$ & $a_{1} z$ & $b_{1} z$ & $b_{1}=c$ \\
\hline$\left(\frac{3}{2} ; 1\right)$ & $z^{2}$ & $a_{1} z$ & $b_{-1} z^{-1}$ & $b_{-1}=c$ \\
\hline$(\underline{1} ; 3)$ & $z$ & $a_{2} z^{2}+a_{1} z+a_{0}$ & $\begin{array}{l}\text { a) } b_{1} z+b_{0} \\
\text { b) } b_{0}+b_{-1} z^{-1}\end{array}$ & $a_{2}=c$ \\
\hline$(3 ; \underline{1})$ & $z^{3}$ & $a_{2} z^{2}+a_{1} z+a_{0}$ & $\begin{array}{l}\text { a) } b_{1} z+b_{0} \\
\text { b) } b_{0}+b_{-1} z^{-1}\end{array}$ & $a_{0}=c$ \\
\hline$\left(\underline{1} ; \frac{5}{2}\right)$ & $z$ & $a_{0}$ & $b_{2} z^{2}+b_{1} z+b_{0}$ & $b_{2}=c$ \\
\hline$\left(\frac{5}{2} ; \underline{1}\right)$ & $z^{3}$ & $a_{2} z^{2}$ & $b_{0}+b_{-1} z^{-1}+b_{-2} z^{-2}$ & $b_{-2}=c$ \\
\hline$(; 4)$ & 1 & $a_{2} z^{2}+a_{0}$ & $b_{1} z+b_{0}$ & $a_{2}=c$ \\
\hline$\left(; \frac{7}{2}\right)$ & 1 & 0 & $b_{3} z^{3}+b_{1} z+b_{0}$ & $b_{3}=c$ \\
\hline
\end{tabular}

In the above table $c$ denotes an arbitrary nonzero constant.

Proof. If $\sigma$ has 3 distinct roots, it can be transformed to $z(z-1)(z-t), t \neq 0,1$. By sandwiching with powers at each finite singularity we can make one of indices 0 . Then $\eta$ becomes a polynomial and $\operatorname{deg} \eta \leq 1$. We obtain the normal form of $(\underline{111} ; \underline{1})$.

Let $\sigma$ have degree 2 and 2 distinct roots. It can be transformed to $z(z-1)$. At each finite singularity we can make one of indices 0 . Then $\eta$ becomes a polynomial and $\operatorname{deg} \eta \leq 2$. By the transformation $\mathrm{e}^{-\kappa z} \cdot \mathrm{e}^{\kappa z}$ with $\kappa$ solving

$$
\kappa^{2}+a_{2} \kappa+b_{2}=0
$$

we can make $b_{2}=0$. If $a_{2} \neq 0$ we obtain the normal form of $(\underline{11} ; 2)$.

Assume that $a_{2}=0$. If $b_{1}=0$, we get the ${ }_{2} F_{1}$ operator, which belongs to the Riemann class. Otherwise we obtain the normal form of $\left(\underline{11} ; \frac{3}{2}\right)$.

Let $\sigma$ have degree 2 and one root. It can be transformed to $z^{2}$. We have

$$
\eta(z)=b_{2} z^{2}+b_{1} z+b_{0}+b_{-1} z^{-1}+b_{-2} z^{-2} .
$$

By $\mathrm{e}^{-\kappa z} \cdot \mathrm{e}^{\kappa z}$ with $\kappa$ solving

$$
\kappa^{2}+a_{2} \kappa+b_{2}=0
$$

we can kill $b_{2}$. By $\mathrm{e}^{\kappa z^{-1}} \cdot \mathrm{e}^{-\kappa z^{-1}}$ with $\kappa$ solving

$$
\kappa^{2}+a_{0} \kappa+b_{-2}=0
$$

we can kill $b_{-2}$. 
Let $a_{0} \neq 0$. By scaling we can make $a_{0}=1$. Then by $z^{-\lambda} \cdot z^{\lambda}$ with $\lambda=-b_{-1}$ we can kill $b_{-1}$, keeping $a_{0}=1$. If $a_{2} \neq 0$, we obtain the normal form of $(2 ; 2)$. If $a_{2}=0$ and $b_{1}=0$, we obtain ${ }_{2} F_{0}$ or Euler II type, both of the Riemann class. If $a_{2}=0$ and $b_{1} \neq 0$, we obtain the normal form of $\left(2 ; \frac{3}{2}\right)$.

Let $a_{0}=0$. If $a_{2} \neq 0$, by scaling we can make $a_{2}=1$ Then by $z^{-\lambda} \cdot z^{\lambda}$ with $\lambda=-b_{1}$ we can kill $b_{1}$ keeping $a_{2}=1$. We obtain the normal form of $\left(\frac{3}{2} ; 2\right)$.

Let $a_{0}=a_{2}=0$. If $b_{1}=b_{-1}=0$, the operator is of the Riemann class. If $b_{-1}=0, b_{1} \neq 0$, then with $z^{-\lambda} \cdot z^{\lambda}$ we kill $b_{0}$ and we obtain $z\left(z \partial_{z}^{2}+a_{1} \partial_{z}+b_{1}\right)$. The operator in brackets can be reduced to the $F_{1}$ operator. If $b_{1}=0, b_{-1} \neq 0$, we similarly kill $b_{0}$ obtaining $\frac{1}{z}\left(z^{3} \partial_{z}^{2}+a_{1} z^{2} \partial_{z}+b_{-1}\right)$. The operator in brackets, after the transformation $z \mapsto \frac{1}{z}$ can be transformed to a $F_{1}$ operator. If $b_{-1}, b_{1} \neq 0$ we apply $z^{-\lambda} \cdot z^{\lambda}$ with $\lambda=-\frac{a_{1}}{2}$ to kill $a_{1}$. We obtain the normal form of $\left(\frac{3}{2} ; \frac{3}{2}\right)$.

Let $\sigma$ have degree 1 . It can be transformed to $z$. One of indices at 0 can be made 0 . Then $\eta$ becomes a polynomial of degree $\leq 3$. By applying $\mathrm{e}^{-\kappa z^{2}} \cdot \mathrm{e}^{\kappa z^{2}}$ with $\kappa=\frac{\sqrt{-b_{3}}}{2}$ we can kill $b_{3}$. If $a_{2} \neq 0$, applying $\mathrm{e}^{-\kappa z} \cdot \mathrm{e}^{\kappa z}$ with $\kappa=-\frac{b_{2}}{a_{2}}$ we kill $b_{2}$. By scaling we can make $a_{2}=1$ and we obtain the normal form of $(\underline{1} ; 3)$. If $a_{2}=0$ and $b_{2} \neq 0$, by applying $\mathrm{e}^{-\kappa z} \cdot \mathrm{e}^{\kappa z}$ with $\kappa=-a_{1}$ we kill $a_{1}$. If $b_{2} \neq 0$, by scaling we can make $b_{2}=1$ and we obtain the normal form of $\left(\underline{1} ; \frac{5}{2}\right)$. If $b_{2}=0$, by applying $\mathrm{e}^{-\kappa z} \cdot \mathrm{e}^{\kappa z}$ with $\kappa$ solving

$$
\kappa^{2}+a_{1} \kappa+b_{1}=0
$$

we obtain an operator which can degenerate to the ${ }_{1} F_{1},{ }_{0} F_{1}$ or Euler I type, all of the Riemann class.

Let $\sigma$ have degree 0 . We can assume that it is $1 . \eta$ is a polynomial of degree 4 .

By applying $\mathrm{e}^{-\kappa z^{3}} \cdot \mathrm{e}^{\kappa z^{3}}$ with $9 \kappa^{2}+a_{2} 3 \kappa+b_{4}=0$ we kill $b_{4}$.

Let $a_{2} \neq 0$. By applying $\mathrm{e}^{-\kappa z^{2}} \cdot \mathrm{e}^{\kappa z^{2}}$ with $\kappa=-\frac{b_{3}}{2 a_{2}}$ we kill $b_{3}$. By applying $\mathrm{e}^{-\kappa z} \cdot \mathrm{e}^{\kappa z}$ with $\kappa=-\frac{b_{2}}{a_{2}}$ we kill $b_{2}$. After a transformation $z \mapsto a z+b$ we can assume that $\tau(z)=z^{2}+a_{0}$. We obtain the normal form of $(; 4)$

Let $a_{2}=0$. By applying $\mathrm{e}^{-\kappa z^{2}} \cdot \mathrm{e}^{\kappa z^{2}}$ with $\kappa=-\frac{a_{1}}{4}$ we kill $a_{1}$. By applying $\mathrm{e}^{-\kappa z} \cdot \mathrm{e}^{\kappa z}$ with $\kappa=-\frac{a_{0}}{2}$ we kill $a_{0}$. Thus $\tau=0$. If $b_{3} \neq 0$, then after a transformation $z \mapsto a z+b$ we can assume that $\tau(z)=z^{3}+a_{1} z+a_{0}$. We obtain the normal form of $\left(; \frac{7}{2}\right)$. If $b_{3}=0$, we obtain an operator that can degenerate to the Hermite, Airy, 1d Helmholtz or 1d Laplace type, all of the Riemann class.

In $(\underline{11} ; 2),(\underline{1} ; 3)$ and $(2 ; 2)$ the transformation $z^{\lambda} \cdot z^{-\lambda}$ with $\lambda=-\frac{b_{1}}{a_{2}}$ kills $b_{1} z$ and produces $b_{-1} z^{-1}$. Thus it makes the normal form b) out of the normal form a).

If $\sigma$ has degree 3 and 2 distinct roots, it can be transformed to $z^{2}(z-1)$. The transformation $z \mapsto z^{-1}$ leads to $\sigma(z)=z(z-1)$.

If $\sigma$ has degree 3 and only 1 root, it can be transformed to $z^{3}$. Then $z \mapsto z^{-1}$ yields $\sigma(z)=z$.

Remark 3.7. The operators listed in the table of Theorem 3.6 as $\left(1 ; \frac{3}{2}\right)$ and $\left(\frac{3}{2} ; 1\right)$ are strictly speaking not of the Riemann class: they are $z$ times an operators of the Riemann class. Hence they yield equations of the Riemann class. So they can be considered as "trivial" and were ignored in the table at the beginning of the subsection.

\section{From Heun class to Painlevé equations}

\subsection{Method of isomonodromic deformations}

Let us review the theory of isomonodromic deformations of linear second order differential equations following $[12,19,20]$. We shall use a notation similar to [19]. 
Let $p, q$ be rational functions of $z$, depending on some parameters. Among these parameters we single out a parameter $t$. We will write $v^{\prime}$ for $\frac{\partial}{\partial z} v$ and $\dot{v}$ for $\frac{\partial}{\partial t} v$. Consider a family of linear second order differential equations of the form

$$
v^{\prime \prime}(z)+p(z) v^{\prime}(z)+q(z) v(z)=0 .
$$

We assume that when we deform the equation (4.1), we can also deform its certain solution $v$ so that the following condition is satisfied:

$$
\dot{v}=a(z, t) v^{\prime}+b(z, t) v .
$$

This essentially means that when we deform the equation, its solutions "live" on the same Riemann surface. In particular, if there are singularities, then one should expect that the monodromy of solutions stays the same.

The compatibility of (4.1) and (4.2) imposes a strong condition on the deformation. Indeed, differentiating (4.2) in $z$ we obtain

$$
\dot{v}^{\prime}=\left(a^{\prime}-a p+b\right) v^{\prime}+\left(-a q+b^{\prime}\right) v .
$$

Differentiating (4.1) once in $t$ and (4.2) twice in $z$ we get

$$
\begin{aligned}
& \dot{v}^{\prime \prime}=\left(-\dot{p}-p a^{\prime}+a p^{2}-p b-q a\right) v^{\prime}+\left(p a q-p b^{\prime}-\dot{q}-q b\right) v, \\
& \dot{v}^{\prime \prime}=\left(-2 q a^{\prime}-a q^{\prime}+b^{\prime \prime}+a p q-b q\right) v+\left(a^{\prime \prime}-2 p a^{\prime}-a p^{\prime}+2 b^{\prime}-a q+a p^{2}-b p\right) v^{\prime} .
\end{aligned}
$$

Equating (4.4) and (4.3) we obtain

$$
\begin{aligned}
& \dot{p}-a p^{\prime}+2 b^{\prime}-p a^{\prime}+a^{\prime \prime}=0, \\
& \dot{q}+p b^{\prime}-2 q a^{\prime}-a q^{\prime}+b^{\prime \prime}=0 .
\end{aligned}
$$

When applying this method to a concrete family of equations one needs to divide its parameters into two categories. The first category should contain all parameters responsible for the monodromy around singular points. For example, the coefficients $p_{-1}$ and $q_{-2}$ of the Laurent series of $p$, resp. $q$ around singular points. In the second category we have parameters that do not influence the monodromy, typically denoted $\mu, \lambda, t$. The variable $t$ is called the "time variable".

\subsection{Isomonodromic deformations in presence of a non-logarithmic singularity}

Let $\sigma, \tau, \eta$ be rational functions. (At the moment we do not assume the conditions (3.15) for the Heun class). Consider the differential equation given by

$$
\partial_{z}^{2}+p_{0}(z) \partial_{z}+q_{0}(z)=\partial_{z}^{2}+\frac{\tau(z)}{\sigma(z)} \partial_{z}+\frac{\eta(z)}{\sigma(z)} .
$$

We assume that $\sigma, \tau, \eta$ depend on a parameter $t$. Let $\lambda, \mu$ be additional parameters. Following the prescription of (2.28), we introduce the deformed equation corresponding to (4.7):

$$
\begin{aligned}
\partial_{z}^{2}+ & p(\lambda, z) \partial_{z}+q(\lambda, \mu, z)=\partial_{z}^{2}+\left(\frac{\tau(z)}{\sigma(z)}-\frac{1}{z-\lambda}\right) \partial_{z} \\
& +\frac{1}{\sigma(z)}\left(\eta(z)-\eta(\lambda)-\mu\left(\tau(\lambda)-\sigma^{\prime}(\lambda)\right)-\mu^{2} \sigma(\lambda)+\frac{\mu \sigma(\lambda)}{(z-\lambda)}\right) .
\end{aligned}
$$

The following theorem is devoted to monodromic deformations of (4.8). It unifies a large family of cases in a single formulation. Unfortunately, this unification has one drawback: relatively complicated conditions (4.9), (4.10) and (4.11) constraining the choice of the time variable $t$ and the auxiliary polynomial $c$. Probably this drawback is impossible to avoid. The main results of our paper, described in the next subsection, will be corollaries of Theorem 4.1. 
Theorem 4.1. Suppose that $c(z)=c(t, \lambda, z)$ is a $t, \lambda$-dependent polynomial of degree $\leq 2$. Suppose that the following conditions are satisfied:

$$
\begin{aligned}
0= & \frac{\dot{\tau}}{\sigma}(z)-\frac{\tau \dot{\sigma}}{\sigma^{2}}(z)+\frac{\frac{c \tau}{\sigma}(z)-\frac{c \tau}{\sigma}(\lambda)-(z-\lambda)\left(\frac{c \tau}{\sigma}\right)^{\prime}(z)}{(z-\lambda)^{2}}, \\
0= & -\frac{\dot{\sigma}}{\sigma}(z)(\eta(z)-\eta(\lambda))+\dot{\eta}(z)-\dot{\eta}(\lambda) \\
& +\frac{\eta(z)-\eta(\lambda)}{(z-\lambda)}\left(\frac{c \sigma^{\prime}}{\sigma}(z)-c^{\prime}(z)\right)-\eta^{\prime}(\lambda)\left(\frac{\sigma^{\prime} c}{\sigma}(\lambda)-c^{\prime}(\lambda)\right) \\
& +\frac{2 c \eta(z)-2 c \eta(\lambda)-\left((c \eta)^{\prime}(z)+(c \eta)^{\prime}(\lambda)\right)(z-\lambda)}{(z-\lambda)^{2}}, \\
0= & \frac{\dot{\sigma}}{\sigma}(z)-\frac{\dot{\sigma}}{\sigma}(\lambda)-\frac{\frac{c \sigma^{\prime}}{\sigma}(z)-\frac{c \sigma^{\prime}}{\sigma}(\lambda)-\left(\frac{c \sigma^{\prime}}{\sigma}\right)^{\prime}(\lambda)(z-\lambda)}{(z-\lambda)} .
\end{aligned}
$$

Define the compatibility functions

$$
a(t, \lambda, z):=\frac{c(z)}{z-\lambda}, \quad b(t, \lambda, \mu, z)=-\frac{c(\lambda) \mu}{z-\lambda} .
$$

Then the following equations for $\lambda, \mu$

$$
\begin{aligned}
& \dot{\lambda}=2 c(\lambda) \mu-c^{\prime}(\lambda)+\frac{c \tau}{\sigma}(\lambda), \\
& \dot{\mu}=-\frac{c \eta^{\prime}}{\sigma}(\lambda)-\mu\left(\frac{c \tau^{\prime}}{\sigma}(\lambda)-\frac{c \sigma^{\prime \prime}}{2 \sigma}(\lambda)-\frac{c^{\prime \prime}}{2}\right)-\mu^{2} \frac{\sigma^{\prime} c}{\sigma}(\lambda)
\end{aligned}
$$

are equivalent to the compatibility conditions (4.6) and (4.5).

The proof of Theorem 4.1 is deferred to Appendixes A.1, A.2 and A.3.

\subsection{Isomonodromic deformations of Heun class equations}

This subsection contains the main results of our paper. We will suppose that $\sigma$ is a polynomial of degree $\leq 3, \tau$ is a polynomial of degree $\leq 2$ and $\eta \sigma$ is a polynomial of degree $\leq 4$. In other words, we will assume that (4.7) is a Heun class equation. We will show that Theorem 4.1 can be applied to a large family of Heun class equations, including normal forms of all its types. As a result we obtain all types of Painlevé equation.

Our main results will be formulated in two theorems. In Theorem 4.2 we still try to give a unified treatment. More precisely, we consider two closely related ansatzes, which we call A and B. Ansatz A is applicable if $\sigma$ has a zero. Ansatz B can be used if the degree of $\sigma$ is $\leq 2$. The time variable is not specified, it is only constrained by certain conditions.

In Theorem 4.3 the time variable is always explicit. Unfortunately, it seems impossible to do it in a unified way - we are compelled to consider 5 distinct cases. (Note that 5 is still less than the number of types of Heun class equations. Besides, some of these cases are applicable to more than one type).

Theorem 4.2. Case A. Assume that $s \in \mathbb{C}$ and $\sigma(s)=0$, so that we can write $\sigma(z)=(z-s) \rho(z)$ for a polynomial $\rho$ of degree $\leq 2$. We assume that $\sigma, \tau, \eta$ depend on $t$. Let $m$ be a function of $t$ satisfying the following conditions

$$
\partial_{t} \frac{\tau}{\sigma}(z)=\frac{m \tau(s)}{(z-s)^{2}}
$$




$$
\begin{aligned}
& \frac{\dot{\sigma}}{\sigma}(z)(\eta(z)-\eta(\lambda))- \\
& \quad \dot{\eta}(z)+\dot{\eta}(\lambda)=m\left(\frac{(\eta(z)-\eta(\lambda))(\lambda-s) \rho(z)}{(z-\lambda)(z-s)}-\eta^{\prime}(\lambda) \rho(\lambda)\right) \\
& +\frac{(\lambda-s)}{(z-\lambda)^{2}}\left(2 \rho \eta(z)-2 \rho \eta(\lambda)-\left((\rho \eta)^{\prime}(z)+(\rho \eta)^{\prime}(\lambda)\right)(z-\lambda)\right), \\
& \frac{\dot{\sigma}}{\sigma}(z)-\frac{\dot{\sigma}}{\sigma}(\lambda)=m \rho(s) \frac{(z-\lambda)}{(z-s)(\lambda-s)} .
\end{aligned}
$$

Define the compatibility functions

$$
a(t, \lambda, z)=\frac{m(\lambda-s) \rho(z)}{z-\lambda}, \quad b(t, \lambda, z)=-\frac{m(\lambda-s) \rho(\lambda) \mu}{z-\lambda},
$$

and the Hamiltonian

$$
H(t, \lambda, \mu)=m\left(\eta(\lambda)+\left(\tau(\lambda)-(\lambda-s) \rho^{\prime}(\lambda)\right) \mu+\sigma(\lambda) \mu^{2}\right) .
$$

Then $\lambda, \mu$ satisfy the Hamilton equations with respect to $H$, that is

$$
\begin{aligned}
\frac{\mathrm{d} \lambda}{\mathrm{d} t} & =\frac{\partial H}{\partial \mu}(t, \lambda, \mu), \\
\frac{\mathrm{d} \mu}{\mathrm{d} t} & =-\frac{\partial H}{\partial \lambda}(t, \lambda, \mu),
\end{aligned}
$$

if and only if (4.6) and (4.5) hold.

Case B. Assume that $\operatorname{deg} \sigma \leq 2$ and $\operatorname{deg} \sigma \eta \leq 3$. Suppose that $\tau$, $\eta$, but not $\sigma$ depend on $t$. Let $m$ be a function of $t$ satisfying

$$
\begin{aligned}
& \frac{\dot{\tau}(z)}{\sigma(z)}=m \frac{\tau^{\prime \prime}}{2} \\
& \dot{\eta}(z)-\dot{\eta}(\lambda)=m \frac{(\sigma \eta)^{\prime \prime \prime}}{6}(z-\lambda) .
\end{aligned}
$$

Define the compatibility functions

$$
a(t, \lambda, z)=\frac{m \sigma(z)}{z-\lambda}, \quad b(t, \lambda, z)=-\frac{m \sigma(\lambda) \mu}{z-\lambda},
$$

and the Hamiltonian

$$
H(t, \lambda, \mu):=m\left(\eta(\lambda)+\left(\tau(\lambda)-\sigma^{\prime}(\lambda)\right) \mu+\sigma(\lambda) \mu^{2}\right) .
$$

Then $\lambda, \mu$ satisfy the Hamilton equations with respect to $H$ if and only if (4.6) and (4.5) hold.

Proof. We set

$$
\begin{aligned}
& c(z)=m(\lambda-s) \rho(z), \quad \text { in Case A, } \\
& c(z)=m \sigma(z), \quad \text { in Case B. }
\end{aligned}
$$

We apply Theorem 4.1. More precisely, we check that conditions (4.9), (4.10) and (4.11) are equivalent to (4.15), (4.16) and (4.17), resp. to (4.19), (4.20). We also verify that equations (4.13) and (4.14) coincide with the Hamilton equations for $H(t, \lambda, \mu)$.

Details of computations are given in Appendixes A.4, A.5 and A.6. 
Note that it is possible to unify the formulas (4.18) and (4.21) for the Hamiltonian in a single formula using the polynomial $c$ from (4.22) and (4.23):

$$
H(t, \lambda, \mu)=\frac{\eta(\lambda) c(\lambda)}{\sigma(\lambda)}+\mu\left(\frac{\tau(\lambda) c(\lambda)}{\sigma(\lambda)}-c^{\prime}(\lambda)\right)+\mu^{2} c(\lambda)
$$

Ansatzes A and B of Theorem 4.2 will still be subdivided into several subcases that differ by the choice of the time variable. All these subcases are described in Theorem 4.3 below, which together with Theorem 4.2 describes the main result of our paper.

The main subcase of Ansatz A is A1, where the time variable is the position of a nondegenerate zero of $\sigma$. This is of course generically true, however there are situations when $\sigma$ does not have nondegenerate zeros. Subcases Ap and Aq can be applied when $\sigma$ has a degenerate (that is, at least double) zero.

In Subcases Ap and Bp time is contained in the function $p_{0}$ (the coefficient of the first order term) of (4.7) and in Aq and Bq it is contained in $q_{0}$. Subcases Ap and Bp are typically used when the rank at $\infty$ is an integer. Subcases $\mathrm{Aq}$ and $\mathrm{Bq}$ are more appropriate for degenerate types, when the rank at $\infty$ is half-integer.

In the following theorem, first we describe $\sigma, \tau$ and $\eta$ that belong to a given subcase, specifying explicitly the dependence on $t$. Next we write the corresponding (undeformed) Heun class operator in the principal form. Then we give the corresponding compatibility functions $a, b$ and the Hamiltonian $H$.

By writing $\operatorname{deg} \beta \leq n$ we mean that $\beta$ is a polynomial in $z$ of degree $\leq n$. Unlike in Theorem 4.3, the dependence on the parameter $t$ will be always explicitly given.

\section{Theorem 4.3.}

- Subcase A1. Let

$$
\begin{aligned}
& \sigma(z)=(z-t) \rho(z), \quad \operatorname{deg} \rho \leq 2 ; \\
& \tau(z)=(1-\kappa) \rho(z)+\phi(z)(z-t), \quad \kappa \in \mathbb{C}, \quad \operatorname{deg} \phi \leq 1 ; \\
& \eta(z)=\frac{\alpha \rho(t)}{z-t}+\eta_{0}(z) \quad \alpha \in \mathbb{C}, \quad \operatorname{deg} \eta_{0} \leq 1 .
\end{aligned}
$$

Consider

$$
\partial_{z}^{2}+\left(\frac{1-\kappa}{z-t}+\frac{\phi(z)}{\rho(z)}\right) \partial_{z}+\frac{\alpha \rho(t)}{(z-t)^{2} \rho(z)}+\frac{\eta_{0}(z)}{(z-t) \rho(z)}
$$

If $\rho(t) \neq 0$, then Theorem $4.2 A$ holds with $m=\rho(t)^{-1}$,

$$
\begin{aligned}
& a(z):=\frac{(\lambda-t) \rho(z)}{\rho(t)(z-\lambda)}, \quad b(z):=-\frac{\sigma(\lambda) \mu}{\rho(t)(z-\lambda)}, \\
& \rho(t) H:=(\lambda-t) \rho(\lambda) \mu^{2}+\left((1-\kappa) \rho(\lambda)+(\lambda-t)\left(\phi(\lambda)-\rho^{\prime}(\lambda)\right) \mu+\frac{\alpha \rho(t)}{\lambda-t}+\eta_{0}(\lambda) .\right.
\end{aligned}
$$

- Subcase Ap. Let

$$
\begin{aligned}
& \sigma(z)=(z-s)^{2} \rho_{1}(z), \quad s \in \mathbb{C}, \quad \operatorname{deg} \rho_{1} \leq 1 \\
& \tau(z)=t \rho_{1}(z)+\tau_{0}(z), \quad \operatorname{deg} \tau_{0} \leq 2 ; \\
& \eta(z)=\frac{\psi(z)}{\rho_{1}(z)}, \quad \operatorname{deg} \psi \leq 2 .
\end{aligned}
$$


Consider

$$
\partial_{z}^{2}+\left(\frac{t}{(z-s)^{2}}+\frac{\tau_{0}(z)}{(z-s)^{2} \rho_{1}(z)}\right) \partial_{z}+\frac{\psi(z)}{(z-s)^{2} \rho_{1}(z)^{2}} .
$$

If $\tau_{0}(s) \neq 0$ or $\rho_{1}(s) \neq 0$, then Theorem $4.2 A$ holds with $m=\left(\tau_{0}(s)+t \rho_{1}(s)\right)^{-1}$,

$$
\begin{aligned}
& a(z):=\frac{(\lambda-s)(z-s) \rho_{1}(z)}{\left(\tau_{0}(s)+t \rho_{1}(s)\right)(z-\lambda)}, \quad b(z):=-\frac{(\lambda-s)^{2} \rho_{1}(\lambda) \mu}{\left(\tau_{0}(s)+t \rho_{1}(s)\right)(z-\lambda)} \\
& \begin{aligned}
\left(\tau_{0}(s)+t \rho_{1}(s)\right) H:= & (\lambda-s)^{2} \rho_{1}(\lambda) \mu^{2} \\
& \quad\left(t \rho_{1}(\lambda)+\tau_{0}(\lambda)-(\lambda-s) \rho_{1}(\lambda)-(\lambda-s)^{2} \rho_{1}^{\prime}\right) \mu+\frac{\psi(\lambda)}{\rho_{1}(\lambda)} .
\end{aligned}
\end{aligned}
$$

- Subcase Aq. Let

$$
\begin{aligned}
& \sigma(z):=(z-s)^{2} \rho_{1}(z), \quad s \in \mathbb{C}, \quad \operatorname{deg} \rho_{1} \leq 1 ; \\
& \tau(z)=(z-s) \phi(z), \quad \operatorname{deg} \phi \leq 1 ; \\
& \eta(z)=\frac{t}{z-s}+\eta_{0}(z), \quad \operatorname{deg} \rho_{1} \eta_{0} \leq 2 .
\end{aligned}
$$

Consider

$$
\partial_{z}^{2}+\frac{\phi(z)}{(z-s) \rho_{1}(z)} \partial_{z}+\frac{1}{(z-s)^{2} \rho_{1}(z)}\left(\frac{t}{z-s}+\eta_{0}(z)\right)
$$

If $\left(\sigma \eta_{0}\right)^{\prime}(s) \neq 0$ or $\rho_{1}(s) \neq 0$, then Theorem $4.2 A$ holds with $m=\left(\left(\sigma \eta_{0}\right)^{\prime}(s)+\rho_{1}(s) t\right)^{-1}$ :

$$
\begin{gathered}
a(z):=\frac{(\lambda-s)(z-s) \rho_{1}(z)}{\left(\left(\sigma \eta_{0}\right)^{\prime}(s)+\rho_{1}(s) t\right)(z-\lambda)}, \quad b(z):=-\frac{(\lambda-s)^{2} \rho_{1}(\lambda) \mu}{\left(\left(\sigma \eta_{0}\right)^{\prime}(s)+\rho_{1}(s) t\right)(z-\lambda)}, \\
\left(\left(\sigma \eta_{0}\right)^{\prime}(s)+\rho_{1}(s) t\right) H:=(\lambda-s)^{2} \rho_{1}(\lambda) \mu^{2}+\left((\lambda-s)\left(\phi(\lambda)-\rho_{1}(\lambda)\right)-(\lambda-s)^{2} \rho_{1}^{\prime}\right) \mu \\
+\frac{t}{\lambda-s}+\eta_{0}(\lambda) .
\end{gathered}
$$

- Subcase Bp. Let

$\sigma(z), \quad \operatorname{deg} \sigma \leq 2$

$\tau(z)=t \sigma(z)+\tau_{0}(z), \quad \operatorname{deg} \tau_{0} \leq 2 ;$

$\eta(z), \quad \operatorname{deg} \sigma \eta \leq 2$.

Consider

$$
\partial_{z}^{2}+\left(t+\frac{\tau_{0}(z)}{\sigma(z)}\right) \partial_{z}+\frac{\eta(z)}{\sigma(z)}
$$

If $\sigma^{\prime \prime} \neq 0$ or $\tau_{0}^{\prime \prime} \neq 0$, then Theorem $4.2 B$ holds with $m=\left(t \frac{\sigma^{\prime \prime}}{2}+\frac{\tau_{0}^{\prime \prime}}{2}\right)^{-1}$ :

$$
\begin{aligned}
& a(z):=\frac{\sigma(z)}{\left(t \frac{\sigma^{\prime \prime}}{2}+\frac{\tau_{0}^{\prime \prime}}{2}\right)(z-\lambda)}, \quad b(z):=-\frac{\sigma(\lambda) \mu}{\left(t \frac{\sigma^{\prime \prime}}{2}+\frac{\tau_{0}^{\prime \prime}}{2}\right)(z-\lambda)}, \\
& \left(t \frac{\sigma^{\prime \prime}}{2}+\frac{\tau_{0}^{\prime \prime}}{2}\right) H:=\sigma(\lambda) \mu^{2}+\left(t \sigma(\lambda)+\tau_{0}(\lambda)-\sigma^{\prime}(\lambda)\right) \mu+\eta(\lambda) .
\end{aligned}
$$


- Subcase Bq. Let

$$
\begin{aligned}
& \sigma(z), \quad \operatorname{deg} \sigma \leq 2 ; \\
& \tau(z), \quad \operatorname{deg} \tau \leq 1 ; \\
& \eta(z)=t z+\eta_{0}(z), \quad \operatorname{deg} \sigma \eta_{0} \leq 3 .
\end{aligned}
$$

Consider

$$
\begin{gathered}
\partial_{z}^{2}+\frac{\tau(z)}{\sigma(z)} \partial_{z}+\frac{t z}{\sigma(z)}+\frac{\eta_{0}(z)}{\sigma(z)} . \\
\text { If } \sigma^{\prime \prime} \neq 0 \text { or }\left(\sigma \eta_{0}\right)^{\prime \prime \prime} \neq 0, \text { then Theorem } 4.2 B \text { holds with } m=\left(t \frac{\sigma^{\prime \prime}}{2}+\frac{\left(\sigma \eta_{0}\right)^{\prime \prime \prime}}{6}\right)^{-1}, \\
a(z):=\frac{\sigma(z)}{\left(t \frac{\sigma^{\prime \prime}}{2}+\frac{\left(\sigma \eta_{0}\right)^{\prime \prime \prime}}{6}\right)(z-\lambda)}, \quad b(z):=-\frac{\sigma(\lambda) \mu}{\left(t \frac{\sigma^{\prime \prime}}{2}+\frac{\left(\sigma \eta_{0}\right)^{\prime \prime \prime}}{6}\right)(z-\lambda)}, \\
\left(t \frac{\sigma^{\prime \prime}}{2}+\frac{\left(\sigma \eta_{0}\right)^{\prime \prime \prime}}{6}\right) H:=\sigma(\lambda) \mu^{2}+\left(\tau(\lambda)-\sigma^{\prime}(\lambda)\right) \mu+t \lambda+\eta_{0}(\lambda) .
\end{gathered}
$$

Note that one can deduce Subcases Ap and Aq from Subcases Bp resp. Bq by applying the symmetry described above Proposition 3.5. However, in Appendices A.4, A.5 and A.6, where we prove Theorem 4.3, we will give independent proofs of all subcases.

Note also that the union of subcases of Theorem 4.3 does not cover the whole Heun class. However, it covers all appropriately interpreted normal forms listed in Theorem 3.6. This will be further discussed in the following subsection.

Remark 4.4. In our applications we will sometimes use rescaled versions of the above constructions. In fact, if $\epsilon \neq 0$, we replace $t$ with $\epsilon t$ and multiply $a, b, H$ with $\epsilon$, then the above theorem remains true.

\subsection{Correspondence between Heun class and Painlevé equations}

Traditionally, Painlevé equations are divided into 6 types: Painlevé I-VI. However, one can argue that some of their degenerate cases should be treated as separate types.

Thus Painlevé V (5.1) splits into the nondegenerate Painlevé V with $\delta \neq 0$ and the degenerate Painlevé $\mathrm{V}$ with $\delta=0$. We denote the former simply by ndeg-V and the latter by deg-V. One can show that deg-V Painlevé is equivalent to Painlevé $\mathrm{III}^{\prime}$, however it is natural to treat it as a separate type. All that is explained in Section 5.2.

With Painlevé III (5.5) the situation is more complicated. First of all, following various authors, we prefer to use the Painlevé III' equation, which is equivalent to Painlevé III by a simple transformation. Beside the nondegenerate case we have the degenerate case and the doubly degenerate case. We denote them respectively, ndeg-III', deg-III' and ddeg-III'. (Ohyama-Okumura denote them $\left(D_{6}^{(1)}\right),\left(D_{7}^{(1)}\right),\left(D_{8}^{(1)}\right)$.) One can also consider an alternative degenerate case $\gamma \neq 0, \delta=0$, which is however equivalent to deg-III'. (Ohyama-Okumura denotes it $\left(D_{7}^{(1)}\right)-2$.) All of that is explained in Section 5.3.

Finally, it is natural to consider the Painlevé 34 equation (4.40), which can be viewed as a degenerate case of the Painlevé IV equation (5.10). One can show that Painlevé 34 is equivalent to Painlevé II, however it is natural to keep it as a separate type. See Section 5.4 for more comments.

This way we obtain 10 types of Painlevé equations. Recall that we also have 10 types of Heun class equations. In fact, each type of Painlevé can be derived from one of the types of deformed Heun. Here is the list of correspondences: 


\begin{tabular}{|c|c|c|c|c|}
\hline (standard) Heun & $(\underline{111} ; \underline{1})$ & A1 a),b) & Painlevé VI & $(\underline{1111})$ \\
\hline \multirow{2}{*}{ ndeg. confluent Heun } & $(\underline{11} ; 2)$ & A1 a),b), Bp b) & \multirow{2}{*}{ Painlevé ndeg-V } & \multirow{2}{*}{$(\underline{112})$} \\
\hline & $(2 \underline{1} ; \underline{1})$ & A1 a), Ap a) & & \\
\hline \multirow{2}{*}{ deg. confluent Heun } & $\left(\underline{11} ; \frac{3}{2}\right)$ & $\mathrm{A} 1, \mathrm{~Bq}$ & \multirow{2}{*}{ Painlevé deg-V } & \multirow{2}{*}{$\left(\underline{11} \frac{3}{2}\right)$} \\
\hline & $\left(\frac{3}{2} \underline{1} ; \underline{1}\right)$ & $\mathrm{Aq}$ & & \\
\hline db. confluent Heun & $(2 ; 2)$ & Ap a), Bp b) & Painlevé ndeg-III' & $(22)$ \\
\hline \multirow{2}{*}{ deg. db. confluent Heun } & $\left(\frac{3}{2} ; 2\right)$ & $\mathrm{Aq}, \mathrm{Bp}$ & \multirow{2}{*}{ Painlevé deg-III' } & \multirow{2}{*}{$\left(\frac{3}{2} 2\right)$} \\
\hline & $\left(2 ; \frac{3}{2}\right)$ & $\mathrm{Ap}, \mathrm{Bq}$ & & \\
\hline ddeg. db. confluent Heun & $\left(\frac{3}{2} ; \frac{3}{2}\right)$ & $\mathrm{Bq}$ & Painlevé ddeg-III' & $\left(\frac{3}{2} \frac{3}{2}\right)$ \\
\hline \multirow{2}{*}{ ndeg. bi-confluent Heun } & $(\underline{1} ; 3)$ & A1 a),b), Bp a),b) & \multirow{2}{*}{ Painlevé IV } & \multirow{2}{*}{$(\underline{1} 3)$} \\
\hline & $(3 ; \underline{1})$ & Ap b) & & \\
\hline \multirow{2}{*}{ deg. bi-confluent Heun } & $\left(\underline{1} ; \frac{5}{2}\right)$ & $\mathrm{Bq}$ & \multirow{2}{*}{ Painlevé 34} & \multirow{2}{*}{$\left(\underline{1} \frac{5}{2}\right)$} \\
\hline & $\left(\frac{5}{2} ; \underline{1}\right)$ & $\mathrm{Aq}$ & & \\
\hline ndeg. tri-confluent Heun & $(; 4)$ & Bp & Painlevé II & $(4)$ \\
\hline deg. tri-confluent Heun & $\left(; \frac{7}{2}\right)$ & $\mathrm{Bq}$ & Painlevé I & $\left(\frac{7}{2}\right)$ \\
\hline
\end{tabular}

In the first column we give the name of the Heun class type. For typographical reasons we abbreviate "nondegenerate" to ndeg, "degenerate" to deg, "doubly degenerate" to ddeg and "doubly" to db.

In the second column we give the symbol of the type in terms of the ranks of singularities. We also indicate which singularity is at $\infty$. In several cases there are two possibilities - we give both of them.

In the third column we indicate subcases of Theorem 4.3 which can be applied to certain normal forms of a given type. Normal forms are taken from the table in Theorem 3.6. If in that table more than one normal form is given, a) or b) indicates which normal form is considered. (In some cases, the normal forms from Theorem 3.6 need to be slightly modified: When we apply A1 to the form b) of $(\underline{111} ; \underline{1})$ we change the roles of the roots; for $(\underline{11} ; 2)$ and $(\underline{1} ; 3)$ we shift one root from 0 to $t$; finally for $(2 \underline{1} ; \underline{1})$ and $\left(\underline{11} ; \frac{3}{2}\right)$ we shift a root from 1 to $t$.)

In the fourth column we give the name of the Painlevé type that can be obtained by the isomonodromic deformation.

In the fifth column we list the symbol in terms of ranks of singularities without the indication of the position of $\infty$. We will often use it in the sequel as the name of the given type of the Painlevé equation. Thus, e.g., the Painlevé $\left(\frac{3}{2} 2\right)$ equation is an alternative name for the degenerate Painlevé III' equation. We will actually prefer these names to the traditional ones, similarly as Slavyanov-Lay in [25].

Occasionally, we will also use the names for Painlevé equation involving the position of $\infty$. For instance, the Painlevé $\left(\frac{3}{2} ; 2\right)$ equation will mean the form of the degenerate Painlevé III' equation obtained from the Heun $\left(\frac{3}{2} ; 2\right)$ equation. The Painlevé $\left(2 ; \frac{3}{2}\right)$ equation will denote the equation obtained from the Heun $\left(2 ; \frac{3}{2}\right)$ equation. Both forms of Painlevé equation are equivalent.

We will discuss further the classification of Painlevé equations in Section 5, where we will see how to group the 10 types into 5 supertypes, parallel to the grouping of 10 types of Heun class equations into 5 supertypes.

In the following subsections we describe how to obtain all types of Painlevé equations from deformed Heun class equations. First we give the functions $\sigma, \tau, \eta$ describing one of possible normal forms of a given type of the Heun class equation. We indicate explicitly the dependence of $\sigma, \tau, \eta$ on the time variable $t$. Then we present this equation in its principal form. Next we give the corresponding deformed equation. Next we give the compatibility functions $a, b$ and the Painlevé Hamiltonian. Finally, we describe the resulting Painlevé equation. 
Note that the whole procedure is determined by $\sigma, \tau, \eta$, by the choice of the time variable $t$ and the functions $a, b$. The latter are restricted by Theorem 4.3. We always indicate which case of Theorem 4.3 we use.

In our derivations we follow the paper of Ohyama-Okumura [19]. We have slightly changed their notation for some of the parameters. We parametrize the equations by the differences of indices at singular points of the deformed equation. In particular, if the rounded rank at $z_{0}$ is 1 , the parameter is called $\kappa_{z_{0}}$, if the rank is 2 , it is called $\chi_{z_{0}}$ and if the rank is 3 it is called $\theta_{z_{0}}$.

One of the parameters of the initial Heun class equation - the free term in $\eta$ - does not enter in the deformed equation, and therefore is not used by [19]. We denote it simply by $c$.

Note that there is some arbitrariness in the choice of Hamiltonians, where a term depending on $t$, but not on $\lambda, \mu$, can always be added. We always choose Hamiltonians coinciding with those of [19].

If in a given type $\sigma$ has a root of multiplicity 1, one can usually use Case A1 of Theorem 4.3. Following [19], we use it only for Heun (1111)-Painlevé VI. For the other types we use Ap, Aq, $\mathrm{Bp}, \mathrm{Bq}$.

In general, for each type of Heun class we give one derivation. The exception is the type $\left(2 \frac{3}{2}\right)$, where, following again [19], we give two versions of derivations of deg-III': one in the form of $\left(2 ; \frac{3}{2}\right)$, the other in the form of $\left(\frac{3}{2} ; 2\right)$.

\subsection{From Heun $(\underline{111} ; \underline{1})$ to Painlevé VI}

Set

$$
\begin{aligned}
& \sigma(z)=z(z-1)(z-t), \\
& \tau(z)=\left(1-\kappa_{0}\right)(z-1)(z-t)+\left(1-\kappa_{1}\right) z(z-t)+\left(1-\kappa_{t}\right) z(z-1), \\
& \eta(z)=\frac{\left(\left(\kappa_{0}+\kappa_{1}+\kappa_{t}-1\right)^{2}-\kappa_{\infty}^{2}\right) z}{4}-c .
\end{aligned}
$$

Heun $(\underline{111} ; \underline{1})$ equation

$$
\partial_{z}^{2}+\left(\frac{1-\kappa_{0}}{z}+\frac{1-\kappa_{1}}{z-1}+\frac{1-\kappa_{t}}{z-t}\right) \partial_{z}+\frac{1}{z(z-1)(z-t)}\left(\frac{\left(\left(\kappa_{0}+\kappa_{1}+\kappa_{t}-1\right)^{2}-\kappa_{\infty}^{2}\right)}{4} z-c\right) .
$$

Deformed Heun $(\underline{111} ; \underline{1})$ equation

$$
\begin{aligned}
\partial_{z}^{2}+ & \left(\frac{1-\kappa_{0}}{z}+\frac{1-\kappa_{1}}{z-1}+\frac{1-\kappa_{t}}{z-t}-\frac{1}{z-\lambda}\right) \partial_{z} \\
+ & \frac{1}{z(z-1)(z-t)}\left(\frac{\left(\left(\kappa_{0}+\kappa_{1}+\kappa_{t}-1\right)^{2}-\kappa_{\infty}^{2}\right)}{4}(z-\lambda)-\mu^{2} \lambda(\lambda-1)(\lambda-t)\right. \\
& \left.\quad+\left(\kappa_{0}(\lambda-1)(\lambda-t)+\kappa_{1} \lambda(\lambda-t)+\kappa_{t} \lambda(\lambda-1)\right) \mu+\frac{\lambda(\lambda-1)(\lambda-t) \mu}{(z-\lambda)}\right) .
\end{aligned}
$$

Type A1 compatibility functions

$$
a(z)=\frac{(\lambda-t) z(z-1)}{t(t-1)(z-\lambda)}, \quad b(z)=-\frac{\lambda(\lambda-1)(\lambda-t) \mu}{t(t-1)(z-\lambda)} .
$$

Painlevé $(\underline{111} ; \underline{1})$ Hamiltonian

$$
\begin{aligned}
t(t-1) H= & \lambda(\lambda-1)(\lambda-t) \mu^{2}-\left(\kappa_{0}(\lambda-1)(\lambda-t)+\kappa_{1} \lambda(\lambda-t)+\left(\kappa_{t}-1\right) \lambda(\lambda-1)\right) \mu \\
& +\frac{\left(\left(\kappa_{0}+\kappa_{1}+\kappa_{t}-1\right)^{2}-\kappa_{\infty}^{2}\right)(\lambda-t)}{4} .
\end{aligned}
$$


Painlevé $(\underline{111} ; \underline{1})$ equation

$$
\begin{aligned}
\frac{\mathrm{d}^{2} \lambda}{\mathrm{d} t^{2}}= & \frac{1}{2}\left(\frac{1}{\lambda}+\frac{1}{\lambda-1}+\frac{1}{\lambda-t}\right)\left(\frac{\mathrm{d} \lambda}{\mathrm{d} t}\right)^{2}-\left(\frac{1}{t}+\frac{1}{t-1}+\frac{1}{\lambda-t}\right) \frac{\mathrm{d} \lambda}{\mathrm{d} t} \\
& +\frac{\lambda(\lambda-1)(\lambda-t)}{t^{2}(t-1)^{2}}\left(\alpha+\beta \frac{t}{\lambda^{2}}+\gamma \frac{t-1}{(\lambda-1)^{2}}+\delta \frac{t(t-1)}{(\lambda-t)^{2}}\right),
\end{aligned}
$$

where

$$
\alpha=\frac{1}{2} \kappa_{\infty}^{2}, \quad \beta=-\frac{1}{2} \kappa_{0}^{2}, \quad \gamma=\frac{1}{2} \kappa_{1}^{2}, \quad \delta=\frac{1}{2}\left(1-\kappa_{t}^{2}\right) .
$$

The standard name of (4.24) is Painlevé VI equation.

\subsection{From Heun $(2 \underline{1} ; \underline{1})$ to Painlevé V}

Set

$$
\begin{aligned}
& \sigma(z)=(z-1)^{2} z, \\
& \tau(z)=\left(2-\chi_{1}\right) z(z-1)+\left(1-\kappa_{0}\right)(z-1)^{2}+t z, \\
& \eta(z)=\frac{\left(\left(\kappa_{0}+\chi_{1}-1\right)^{2}-\kappa_{\infty}^{2}\right)(z-1)}{4}-c .
\end{aligned}
$$

Heun $(2 \underline{1} ; \underline{1})$ equation (with the singularity of rank 2 put at 1 ):

$$
\partial_{z}^{2}+\left(\frac{2-\chi_{1}}{z-1}+\frac{1-\kappa_{0}}{z}+\frac{t}{(z-1)^{2}}\right) \partial_{z}+\frac{1}{(z-1)^{2} z}\left(\frac{\left(\kappa_{0}+\chi_{1}-1\right)^{2}-\kappa_{\infty}^{2}}{4}(z-1)-c\right) .
$$

Deformed Heun $(2 \underline{1} ; \underline{1})$ equation:

$$
\begin{aligned}
\partial_{z}^{2}+ & \left(\frac{t}{(z-1)^{2}}+\frac{2-\chi_{1}}{z-1}+\frac{1-\kappa_{0}}{z}-\frac{1}{z-\lambda}\right) \partial_{z} \\
+ & \frac{1}{(z-1)^{2} z}\left(\frac{\left(\kappa_{0}+\chi_{1}-1\right)^{2}-\kappa_{\infty}^{2}}{4}(z-\lambda)-(\lambda-1)^{2} \lambda \mu^{2}\right. \\
& \left.\quad-\left(-\kappa_{0}(\lambda-1)^{2}-\chi_{1} \lambda(\lambda-1)+t \lambda\right) \mu+\frac{(\lambda-1)^{2} \lambda \mu}{(z-\lambda)}\right) .
\end{aligned}
$$

Type Ap compatibility functions

$$
a(z)=\frac{(\lambda-1) z(z-1)}{t(z-\lambda)}, \quad b(z)=-\frac{(\lambda-1)^{2} \lambda \mu}{t(z-\lambda)} .
$$

Painlevé (2푸 $\underline{1})$ Hamiltonian

$$
\begin{aligned}
t H= & (\lambda-1)^{2} \lambda \mu^{2}-\left(\kappa_{0}(\lambda-1)^{2}+\left(\chi_{1}-1\right) \lambda(\lambda-1)-t \lambda\right) \mu \\
& +\frac{\left(\left(\kappa_{0}+\chi_{1}-1\right)^{2}-\kappa_{\infty}^{2}\right)(\lambda-1)}{4} .
\end{aligned}
$$

Painlevé $(2 \underline{1} ; \underline{1})$ equation:

$$
\frac{\mathrm{d}^{2} \lambda}{\mathrm{d} t^{2}}=\left(\frac{1}{2 \lambda}+\frac{1}{\lambda-1}\right)\left(\frac{\mathrm{d} \lambda}{\mathrm{d} t}\right)^{2}-\frac{1}{t} \frac{\mathrm{d} \lambda}{\mathrm{d} t}+\frac{(\lambda-1)^{2}}{t^{2}}\left(\alpha \lambda+\frac{\beta}{\lambda}\right)+\gamma \frac{\lambda}{t}-\frac{\lambda(\lambda+1)}{2(\lambda-1)},
$$

where

$$
\alpha=\frac{1}{2} \kappa_{\infty}^{2}, \quad \beta=-\frac{1}{2} \kappa_{0}^{2}, \quad \gamma=\chi_{1} .
$$

According to the standard terminology, (4.26) is the nondegenerate case of the Painlevé $\mathrm{V}$ equation. 


\subsection{From Heun $\left(\frac{3}{2} \underline{1} ; \underline{1}\right)$ to degenerate Painlevé V}

Set

$$
\begin{aligned}
& \sigma(z)=(z-1)^{2} z \\
& \tau(z)=(z-1) z+\left(1-\kappa_{0}\right)(z-1)^{2}, \\
& \eta(z)=-\frac{t}{(z-1)}+\frac{\left(\kappa_{0}^{2}-\kappa_{\infty}^{2}\right) z}{4}-c .
\end{aligned}
$$

Heun $\left(\frac{3}{2} 1 ; 1\right)$ equation (with the singularity of rank 2 put at 1 ):

$$
\partial_{z}^{2}+\left(\frac{1}{z-1}+\frac{1-\kappa_{0}}{z}\right) \partial_{z}+\frac{1}{z(z-1)^{2}}\left(-\frac{t}{(z-1)}+\frac{\left(\kappa_{0}^{2}-\kappa_{\infty}^{2}\right)}{4}(z-1)-c\right) .
$$

Deformed Heun $\left(\frac{3}{2} \underline{1} ; \underline{1}\right)$ equation:

$$
\begin{aligned}
\partial_{z}^{2}+ & \left(\frac{1}{z-1}+\frac{1-\kappa_{0}}{z}-\frac{1}{z-\lambda}\right) \partial_{z}+\frac{1}{(z-1)^{2} z}\left(-\frac{t}{(z-1)}+\frac{t}{(\lambda-1)}+\frac{\left(\kappa_{0}^{2}-\kappa_{\infty}^{2}\right)}{4}(z-\lambda)\right. \\
& \left.-\lambda(\lambda-1)^{2} \mu^{2}+\left(\lambda(\lambda-1)+\kappa_{0}(\lambda-1)^{2}\right) \mu+\frac{(\lambda-1)^{2} \lambda \mu}{(z-\lambda)}\right) .
\end{aligned}
$$

Type Aq compatibility functions:

$$
a(z)=\frac{(\lambda-1) z(z-1)}{t(z-\lambda)}, \quad b(z)=-\frac{\lambda(\lambda-1)^{2} \mu}{t(z-\lambda)} .
$$

Painlevé $\left(\frac{3}{2} \underline{1} ; \underline{1}\right)$ Hamiltonian:

$$
t H=\lambda(\lambda-1)^{2} \mu^{2}-\kappa_{0}(\lambda-1)^{2} \mu+\frac{\left(\kappa_{0}^{2}-\kappa_{\infty}^{2}\right)(\lambda-1)}{4}-\frac{t \lambda}{(\lambda-1)} .
$$

Painlevé $\left(\frac{3}{2} \underline{1} ; \underline{1}\right)$ equation:

$$
\frac{\mathrm{d}^{2} \lambda}{\mathrm{d} t^{2}}=\left(\frac{1}{2 \lambda}+\frac{1}{\lambda-1}\right)\left(\frac{\mathrm{d} \lambda}{\mathrm{d} t}\right)^{2}-\frac{1}{t} \frac{\mathrm{d} \lambda}{\mathrm{d} t}+\frac{(\lambda-1)^{2}}{t^{2}}\left(\alpha \lambda+\frac{\beta}{\lambda}\right)-2 \frac{\lambda}{t},
$$

where

$$
\alpha=\frac{1}{2} \kappa_{\infty}^{2}, \quad \beta=-\frac{1}{2} \kappa_{0}^{2} .
$$

According to the standard terminology (4.28) is the degenerate Painlevé V equation.

\subsection{From Heun $(2 ; 2)$ to non-degenerate Painlevé III'}

Set

$$
\sigma(z)=z^{2}, \quad \tau(z)=t+\left(2-\chi_{0}\right) z-z^{2}, \quad \eta(z)=\frac{\left(\chi_{0}+\chi_{\infty}-1\right) z}{2}-c .
$$

Heun $(2 ; 2)$ equation:

$$
\partial_{z}^{2}+\left(\frac{t}{z^{2}}+\frac{2-\chi_{0}}{z}-1\right) \partial_{z}+\frac{\left(\chi_{0}+\chi_{\infty}-1\right)}{2 z}-\frac{c}{z^{2}} .
$$


Deformed Heun $(2 ; 2)$ equation:

$$
\begin{aligned}
\partial_{z}^{2}+ & \left(\frac{t}{z^{2}}+\frac{2-\chi_{0}}{z}-1-\frac{1}{z-\lambda}\right) \partial_{z} \\
& +\frac{1}{z^{2}}\left(\frac{\left(\chi_{0}+\chi_{\infty}-1\right)}{2}(z-\lambda)-\lambda^{2} \mu^{2}-\left(t-\chi_{0} \lambda-\lambda^{2}\right) \mu+\frac{\lambda^{2} \mu}{(z-\lambda)}\right) .
\end{aligned}
$$

Type Ap compatibility functions:

$$
a(z):=\frac{\lambda z}{t(z-\lambda)}, \quad b(z)=-\frac{\lambda^{2} \mu}{t(z-\lambda)} .
$$

Painlevé $(2 ; 2)$ Hamiltonian:

$$
t H:=\lambda^{2} \mu^{2}-\left(\lambda^{2}+\left(\chi_{0}-1\right) \lambda-t\right) \mu+\frac{1}{2}\left(\chi_{0}+\chi_{\infty}-1\right) \lambda .
$$

Painlevé $(2 ; 2)$ equation:

$$
\frac{\mathrm{d}^{2} \lambda}{\mathrm{d} t^{2}}=\frac{1}{\lambda}\left(\frac{\mathrm{d} \lambda}{\mathrm{d} t}\right)^{2}-\frac{1}{t} \frac{\mathrm{d} \lambda}{\mathrm{d} t}+\frac{\alpha \lambda^{2}}{4 t^{2}}+\frac{\lambda^{3}}{t^{2}}+\frac{\beta}{4 t}-\frac{1}{\lambda},
$$

where

$$
\alpha=-4 \chi_{\infty}, \quad \beta=4 \chi_{0} .
$$

According to the standard terminology (4.30) could be called the nondegenerate Painlevé III' equation.

\subsection{From Heun $\left(2 ; \frac{3}{2}\right)$ to degenerate Painlevé III'}

We set

$$
\sigma(z):=z^{2}, \quad \tau(z)=t+z\left(2-\chi_{0}\right), \quad \eta(z)=\frac{z}{2}-c .
$$

Heun $\left(2 ; \frac{3}{2}\right)$ equation:

$$
\partial_{z}^{2}+\left(\frac{t}{z^{2}}+\frac{2-\chi_{0}}{z}\right) \partial_{z}+\frac{1}{z^{2}}\left(\frac{1}{2} z-c\right) .
$$

Deformed Heun $\left(2 ; \frac{3}{2}\right)$ equation:

$$
\partial_{z}^{2}+\left(\frac{t}{z^{2}}+\frac{2-\chi_{0}}{z}-\frac{1}{z-\lambda}\right) \partial_{z}+\frac{1}{z^{2}}\left(\frac{(z-\lambda)}{2}-\lambda^{2} \mu^{2}-\left(t-\chi_{0} \lambda\right) \mu+\frac{\lambda^{2} \mu}{(z-\lambda)}\right) .
$$

Type Ap compatibility functions

$$
a(z):=\frac{\lambda z}{t(z-\lambda)}, \quad b(z)=-\frac{\lambda^{2} \mu}{t(z-\lambda)} .
$$

Painlevé $\left(2 ; \frac{3}{2}\right)$ Hamiltonian:

$$
\left.t H=\lambda^{2} \mu^{2}+\left(1-\chi_{0}\right) \lambda+t\right) \mu+\frac{\lambda}{2} .
$$

Painlevé $\left(2 ; \frac{3}{2}\right)$ equation:

$$
\frac{\mathrm{d}^{2} \lambda}{\mathrm{d} t^{2}}=\frac{1}{\lambda}\left(\frac{\mathrm{d} \lambda}{\mathrm{d} t}\right)^{2}-\frac{1}{t} \frac{\mathrm{d} \lambda}{\mathrm{d} t}-\frac{\lambda^{2}}{t^{2}}+\frac{\beta}{4 t}-\frac{1}{\lambda},
$$

where

$$
\beta=4 \chi_{0} .
$$

According to the standard terminology (4.32) is one of the forms of the degenerate Painlevé III' equation. 


\subsection{From Heun $\left(\frac{3}{2} ; 2\right)$ to degenerate Painlevé III' $^{\prime}$}

Set

$$
\sigma(z)=z^{2}, \quad \tau(z)=-z^{2}+z, \quad \eta(z)=\frac{t}{2 z}-c+\frac{\chi_{\infty} z}{2} .
$$

Heun $\left(\frac{3}{2} ; 2\right)$ equation:

$$
\partial_{z}^{2}+\left(-1+\frac{1}{z}\right) \partial_{z}+\frac{1}{z^{2}}\left(\frac{t}{2 z}-c+\frac{\chi_{\infty}}{2} z\right) .
$$

Deformed Heun $\left(\frac{3}{2} ; 2\right)$ equation:

$$
\partial_{z}^{2}+\left(-1+\frac{1}{z}-\frac{1}{z-\lambda}\right) \partial_{z}+\frac{1}{z^{2}}\left(\frac{t}{2 z}-\frac{t}{2 \lambda}+\frac{\chi_{\infty}}{2}(z-\lambda)-\lambda^{2} \mu^{2}+\left(\lambda^{2}+\lambda\right) \mu+\frac{\mu \lambda^{2}}{(z-\lambda)}\right) .
$$

Type Aq compatibility functions:

$$
a(z):=\frac{\lambda z}{t(z-\lambda)}, \quad b(z)=-\frac{\lambda^{2} \mu}{t(z-\lambda)} .
$$

Painlevé $\left(\frac{3}{2} ; 2\right)$ Hamiltonian

$$
t H=\lambda^{2} \mu^{2}-\lambda^{2} \mu+\frac{\chi_{\infty} \lambda}{2}+\frac{t}{2 \lambda} .
$$

Painlevé $\left(\frac{3}{2} ; 2\right)$ equation:

$$
\frac{\mathrm{d}^{2} \lambda}{\mathrm{d} t^{2}}=\frac{1}{\lambda}\left(\frac{\mathrm{d} \lambda}{\mathrm{d} t}\right)^{2}-\frac{1}{t} \frac{\mathrm{d} \lambda}{\mathrm{d} t}+\frac{\alpha \lambda^{2}}{4 t^{2}}+\frac{\lambda^{3}}{t^{2}}+\frac{1}{t},
$$

where

$$
\alpha=-4 \chi_{\infty}
$$

According to the standard terminology (4.32) is one of the forms of the degenerate Painlevé III' equation.

\subsection{From Heun $\left(\frac{3}{2} ; \frac{3}{2}\right)$ to doubly degenerate Painlevé III'}

We set

$$
\sigma(z)=z^{2}, \quad \tau(z)=2 z, \quad \eta(z)=\frac{z}{2}-c+\frac{t}{2 z} .
$$

Heun $\left(\frac{3}{2} ; \frac{3}{2}\right)$ equation

$$
\partial_{z}^{2}+\frac{2}{z} \partial_{z}+\frac{1}{z^{2}}\left(\frac{1}{2} z-c+\frac{t}{2 z}\right) .
$$

Deformed Heun $\left(\frac{3}{2} ; \frac{3}{2}\right)$ equation:

$$
\partial_{z}^{2}+\left(\frac{2}{z}-\frac{1}{z-\lambda}\right) \partial_{z}+\frac{1}{z^{2}}\left(\frac{t}{2 z}-\frac{t}{2 \lambda}+\frac{1}{2}(z-\lambda)-\lambda^{2} \mu^{2}+\frac{\mu \lambda^{2}}{(z-\lambda)}\right) .
$$


Type Aq compatibility functions:

$$
a(z):=\frac{\lambda z}{t(z-\lambda)}, \quad b(z)=-\frac{\lambda^{2} \mu}{t(z-\lambda)} .
$$

Painlevé $\left(\frac{3}{2} ; \frac{3}{2}\right)$ Hamiltonian

$$
t H=\lambda^{2} \mu^{2}+\lambda \mu+\frac{\lambda}{2}+\frac{t}{2 \lambda} .
$$

Painlevé $\left(\frac{3}{2} ; \frac{3}{2}\right)$ equation:

$$
\frac{\mathrm{d}^{2} \lambda}{\mathrm{d} t^{2}}=\frac{1}{\lambda}\left(\frac{\mathrm{d} \lambda}{\mathrm{d} t}\right)^{2}-\frac{1}{t} \frac{\mathrm{d} \lambda}{\mathrm{d} t}-\frac{\lambda^{2}}{t^{2}}+\frac{1}{t} .
$$

Following the standard terminology (4.36) could be called the doubly degenerate Painlevé III' equation.

\subsection{From Heun $(\underline{1} ; 3)$ to Painlevé IV}

Set

$$
\sigma(z)=z, \quad \tau(z)=1-\kappa_{0}-t z-\frac{z^{2}}{2}, \quad \eta(z)=\frac{\theta_{\infty} z}{2}-c .
$$

Heun $(\underline{1} ; 3)$ equation

$$
\partial_{z}^{2}+\left(\frac{1-\kappa_{0}}{z}-t-\frac{z}{2}\right) \partial_{z}+\frac{1}{z}\left(\frac{\theta_{\infty}}{2} z-c\right) .
$$

Deformed Heun $(\underline{1} ; 3)$ equation

$$
\partial_{z}^{2}+\left(\frac{1-\kappa_{0}}{z}-t-\frac{z}{2}-\frac{1}{z-\lambda}\right) \partial_{z}+\frac{1}{z}\left(\frac{\theta_{\infty}(z-\lambda)}{2}-\lambda \mu^{2}+\left(\frac{1}{2} \lambda^{2}+t \lambda+\kappa_{0}\right) \mu+\frac{\lambda \mu}{(z-\lambda)}\right) .
$$

Type Bp compatibility functions:

$$
a(z):=\frac{2 z}{(z-\lambda)}, \quad b(z)=-\frac{2 \lambda \mu}{(z-\lambda)} .
$$

Painlevé $(\underline{1} ; 3)$ Hamiltonian:

$$
H=2 \lambda \mu^{2}-\left(\lambda^{2}+2 t \lambda+2 \kappa_{0}\right) \mu+\theta_{\infty} \lambda .
$$

Painlevé $(\underline{1} ; 3)$ equation

$$
\frac{\mathrm{d}^{2} \lambda}{\mathrm{d} t^{2}}=\frac{1}{2 \lambda}\left(\frac{\mathrm{d} \lambda}{\mathrm{d} t}\right)^{2}+\frac{3}{2} \lambda^{3}+4 t \lambda^{2}+2\left(t^{2}-\alpha\right) \lambda+\frac{\beta}{\lambda}
$$

where

$$
\alpha=-\kappa_{0}+2 \theta_{\infty}-1, \quad \beta=-2 \kappa_{0}^{2} .
$$

In the standard terminology (4.38) is called the Painlevé IV equation. 


\subsection{From Heun $\left(\underline{1} ; \frac{5}{2}\right)$ to Painlevé 34}

Set

$$
\sigma(z)=z, \quad \tau(z)=1-\kappa_{0}, \quad \eta(z)=-\frac{1}{2} z^{2}-\frac{1}{2} t z-c .
$$

Heun $\left(\underline{1} ; \frac{5}{2}\right)$ equation:

$$
\partial_{z}^{2}+\frac{1-\kappa_{0}}{z} \partial_{z}+\frac{1}{z}\left(-\frac{1}{2} z^{2}-\frac{t z}{2}-c\right) .
$$

Deformed Heun $\left(\underline{1} ; \frac{5}{2}\right)$ equation:

$$
\partial_{z}^{2}+\left(\frac{1-\kappa_{0}}{z}-\frac{1}{z-\lambda}\right)+\frac{1}{z}\left(-\frac{z^{2}}{2}+\frac{\lambda^{2}}{2}-\frac{t}{2}(z-\lambda)-\lambda \mu^{2}+\kappa_{0} \mu+\frac{\lambda \mu}{z-\lambda}\right) .
$$

Type Bq compatibility functions:

$$
a(z)=\frac{z}{z-\lambda}, \quad b(z)=-\frac{\lambda \mu}{z-\lambda} .
$$

Painlevé $\left(\underline{1} ; \frac{5}{2}\right)$ Hamiltonian

$$
H=\lambda \mu^{2}-\kappa_{0} \mu-\frac{\lambda^{2}}{2}-\frac{t \lambda}{2} .
$$

Painlevé $\left(\underline{1} ; \frac{5}{2}\right)$ equation:

$$
\frac{\mathrm{d}^{2} \lambda}{\mathrm{d} t^{2}}=\frac{1}{2 \lambda}\left(\frac{\mathrm{d} \lambda}{\mathrm{d} t}\right)^{2}+2 \lambda^{2}+t \lambda-\frac{\alpha}{2 \lambda}
$$

where $\alpha=\kappa_{0}^{2}$.

According to [19], the standard name for (4.40) is the Painlevé 34 equation.

\subsection{From Heun $(; 4)$ to Painlevé II}

Set

$$
\sigma(z)=1, \quad \tau(z)=-2 z^{2}-t, \quad \eta(z)=-(2 \alpha+1) z-c .
$$

Heun $(; 4)$ equation:

$$
\partial_{z}^{2}-\left(2 z^{2}+t\right) \partial_{z}-(2 \alpha+1) z-c
$$

Deformed Heun (; 4) equation:

$$
\partial_{z}^{2}+\left(-2 z^{2}-t-\frac{1}{z-\lambda}\right) \partial_{z}-(2 \alpha+1)(z-\lambda)-\mu^{2}+\left(2 \lambda^{2}+t\right) \mu+\frac{\mu}{z-\lambda} .
$$

Type Bp compatibility functions scaled with $\epsilon=-1$ :

$$
a(z):=\frac{1}{2(z-\lambda)}, \quad b(z)=-\frac{\mu}{2(z-\lambda)} .
$$

Painlevé (;4) Hamiltonian:

$$
H=\frac{1}{2} \mu^{2}-\left(\lambda^{2}+\frac{t}{2}\right) \mu-\left(\alpha+\frac{1}{2}\right) \lambda .
$$

Painlevé $(; 4)$ equation:

$$
\frac{\mathrm{d}^{2} \lambda}{\mathrm{d} t^{2}}=2 \lambda^{3}+t \lambda+\alpha
$$

According to the standard terminology (4.42) is called the Painlevé II equation 


\subsection{From Heun $\left(; \frac{7}{2}\right)$ to Painlevé I}

Set

$$
\sigma(z)=1, \quad \tau(z)=0, \quad \eta(z)=-4 z^{3}-2 t z-c .
$$

Heun $\left(; \frac{7}{2}\right)$ equation:

$$
\partial_{z}^{2}-4 z^{3}-2 t z-c .
$$

Deformed Heun $\left(; \frac{7}{2}\right)$ equation:

$$
\partial_{z}^{2}-\frac{1}{(z-\lambda)} \partial_{z}-4 z^{3}-2 t z+4 \lambda^{3}+2 t \lambda-\mu^{2}+\frac{\mu}{(z-\lambda)} .
$$

Type Bq compatibility functions scaled with $\epsilon=-6$ :

$$
a(z):=\frac{1}{2(z-\lambda)}, \quad b(z)=-\frac{\mu}{2(z-\lambda)} .
$$

Painlevé (; $\left.\frac{7}{2}\right)$ Hamiltonian:

$$
H=\frac{1}{2} \mu^{2}-2 \lambda^{3}-t \lambda .
$$

Painlevé $\left(; \frac{7}{2}\right)$ equation:

$$
\frac{\mathrm{d}^{2} \lambda}{\mathrm{d} t^{2}}=6 \lambda^{2}+t
$$

In the standard terminology (4.44) is called the Painlevé I equation.

\section{$5 \quad$ Five supertypes of Painlevé equation}

\subsection{Overview of five supertypes}

Recall that the ten types of the Heun class equation can be grouped into five supertypes, as described in Section 3.6. The ten types of Painlevé equation can be also grouped into five supertypes. There is an exact correspondence between the supertypes of Heun class and Painlevé equations:

- Painlevé VI or (1111).

- Painlevé V or $(\underline{112})$.

- Painlevé ndeg-V or (112).

- Painlevé deg-V or $\left(\underline{11} \frac{3}{2}\right)$.

- Painlevé III' or (222).

- Painlevé ndeg-III' or (22).

- Painlevé deg-III' or $\left(\frac{3}{2} 2\right)$.

- Painlevé ddeg-III' or $\left(\frac{3}{2} \frac{3}{2}\right)$.

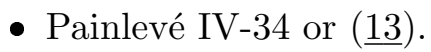

- Painlevé IV or (13). 
- Painlevé 34 or $\left(\underline{1} \frac{5}{2}\right)$.

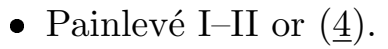

- Painlevé II or (4).

- Painlevé I or $\left(\frac{7}{2}\right)$.

In what follows we discuss this classification. We describe the minimal set of parameters that can be used in a given type and various equivalences. In our discussion we try to include the Hamiltonian aspect, whenever it is possible.

The above classification of Painlevé equation was pointed out by Ohyama-Okumura, see the beginning of Section 2 of [19]. (In that reference the authors use the word "type" both for what we call "supertype" and "type".) The discussion in [19], however, concentrated on the second order equations. Less space was devoted to the Hamiltonian form of the five supertypes.

The first supertype is Painlevé VI or (1111), which contains only one type. All the four remaining supertypes contain at least two types. We discuss them in the following subsections.

In each of the following subsections we start with a general form of the given supertype of the Painlevé equation. It will be indicated by $\diamond$. It always has the form

$$
\frac{\mathrm{d}^{2} \lambda}{\mathrm{d} t^{2}}=F\left(t, \frac{\mathrm{d} \lambda}{\mathrm{d} t}, \lambda\right)
$$

The corresponding differential (nonlinear) operator will be denoted

$$
P(t, \lambda):=\frac{\mathrm{d}^{2} \lambda}{\mathrm{d} t^{2}}-F\left(t, \frac{\mathrm{d} \lambda}{\mathrm{d} t}, \lambda\right)
$$

We also introduce the corresponding Hamiltonian $H(t, \lambda, \mu)$. Both $P(t, \lambda)$ and $H(t, \lambda, \mu)$ depend on several parameters, put as subscripts. Next we give the scaling properties of the equation and the Hamiltonian.

Then we list various nontrivial types that belong to a given supertype, marking them with $*$.

Finally, we discuss the relationship between various types. In particular, show how to reduce the number of parameters using scaling.

Each supertype contains one generic type, which we call non-degenerate. Besides, it may contain one or more degenerate types. The Hamiltonian that covers the non-degenerate type, does not always allow us to describe all types that belong to a given supertype. This can be viewed as a drawback of the Hamiltonian approach.

\subsection{Painlevé V or $(\underline{\mathbf{1 1 2}})$}

As noted in [19], the usual form of the Painlevé V equation, depending on 4 parameters, should be treated not as a single type, but as a supertype. It is invariant with respect to a scaling transformation. It includes two nontrivial types: nondegenerate $\mathrm{V}$ depending on 3 parameters and degenerate $\mathrm{V}$ depending on 2 parameters. There exists also a trivial type, solvable in quadrature.

In this subsection we discuss the supertype Painlevé $\mathrm{V}$ in detail. Note that we prefer to denote it by $(\underline{112})$, since it corresponds to the supertype (112) of the Heun class.

$\diamond$ Painlevé V or (112) equation and Hamiltonian

$$
\begin{aligned}
& \frac{\mathrm{d}^{2} \lambda}{\mathrm{d} t^{2}}=\left(\frac{1}{2 \lambda}+\frac{1}{\lambda-1}\right)\left(\frac{\mathrm{d} \lambda}{\mathrm{d} t}\right)^{2}-\frac{1}{t} \frac{\mathrm{d} \lambda}{\mathrm{d} t}+\frac{(\lambda-1)^{2}}{t^{2}}\left(\alpha \lambda+\frac{\beta}{\lambda}\right)+\gamma \frac{\lambda}{t}+\delta \frac{\lambda(\lambda+1)}{\lambda-1}, \\
& t H=(\lambda-1)^{2} \lambda \mu^{2}-\left(\kappa_{0}(\lambda-1)^{2}+\left(\chi_{1}-1\right) \lambda(\lambda-1)-\eta t \lambda\right) \mu
\end{aligned}
$$




$$
+\frac{\left(\left(\kappa_{0}+\chi_{1}-1\right)^{2}-\kappa_{\infty}^{2}\right)(\lambda-1)}{4}
$$

where $\alpha=\frac{1}{2} \kappa_{\infty}^{2}, \beta=-\frac{1}{2} \kappa_{0}^{2}, \gamma=\chi_{1} \eta, \delta=-\frac{1}{2} \eta^{2}$.

Scaling properties

$$
\begin{aligned}
& \epsilon^{2} P_{\alpha, \beta, \gamma, \delta}(\epsilon t, \lambda)=P_{\alpha, \beta, \epsilon \gamma, \epsilon^{2} \delta}(t, \lambda), \\
& \epsilon H_{\kappa_{0}, \kappa_{\infty}, \chi_{1}, \eta}(\epsilon t, \lambda, \mu)=H_{\kappa_{0}, \kappa_{\infty}, \chi_{1}, \frac{\eta}{\epsilon}}(t, \lambda, \mu) .
\end{aligned}
$$

* Painlevé ndeg-V or (211) equation and Hamiltonian recalled from (4.26) and (4.25):

$$
\begin{aligned}
\frac{\mathrm{d}^{2} \lambda}{\mathrm{d} t^{2}}= & \left(\frac{1}{2 \lambda}+\frac{1}{\lambda-1}\right)\left(\frac{\mathrm{d} \lambda}{\mathrm{d} t}\right)^{2}-\frac{1}{t} \frac{\mathrm{d} \lambda}{\mathrm{d} t}+\frac{(\lambda-1)^{2}}{t^{2}}\left(\alpha \lambda+\frac{\beta}{\lambda}\right)+\gamma \frac{\lambda}{t}-\frac{\lambda(\lambda+1)}{2(\lambda-1)}, \\
t H= & (\lambda-1)^{2} \lambda \mu^{2}-\left(\kappa_{0}(\lambda-1)^{2}+\left(\chi_{1}-1\right) \lambda(\lambda-1)-t \lambda\right) \mu \\
& +\frac{\left(\left(\kappa_{0}+\chi_{1}-1\right)^{2}-\kappa_{\infty}^{2}\right)(\lambda-1)}{4},
\end{aligned}
$$

where $\alpha=\frac{1}{2} \kappa_{\infty}^{2}, \beta=-\frac{1}{2} \kappa_{0}^{2}, \gamma=\chi_{1}$.

* Painlevé deg-V or $\left(\frac{3}{2} \underline{11}\right)$ equation and Hamiltonian, recalled from (4.28) and (4.27):

$$
\begin{aligned}
& \frac{\mathrm{d}^{2} \lambda}{\mathrm{d} t^{2}}=\left(\frac{1}{2 \lambda}+\frac{1}{\lambda-1}\right)\left(\frac{\mathrm{d} \lambda}{\mathrm{d} t}\right)^{2}-\frac{1}{t} \frac{\mathrm{d} \lambda}{\mathrm{d} t}+\frac{(\lambda-1)^{2}}{t^{2}}\left(\alpha \lambda+\frac{\beta}{\lambda}\right)-2 \frac{\lambda}{t}, \\
& t H=\lambda(\lambda-1)^{2} \mu^{2}-\kappa_{0}(\lambda-1)^{2} \mu+\frac{\left(\kappa_{0}^{2}-\kappa_{\infty}^{2}\right)(\lambda-1)}{4}-\frac{t \lambda}{(\lambda-1)},
\end{aligned}
$$

where $\alpha=\frac{1}{2} \kappa_{\infty}^{2}, \beta=-\frac{1}{2} \kappa_{0}^{2}$.

Let us discuss special cases:

- Let $\delta \neq 0$. In the Hamiltonian form it corresponds to $\eta \neq 0$. By scaling we can set $\delta=-\frac{1}{2}$, and in the Hamiltonian form $\eta=1$. We obtain the Painlevé (112) equation and Hamiltonian.

- Let $\delta=0, \gamma \neq 0$. By scaling we can set $\gamma=-2$. We obtain the Painlevé $\left(\underline{11} \frac{3}{2}\right)$ equation. However, on the Hamiltonian level this reduction does not work: we cannot directly use (5.2) to obtain the Painlevé $\left(\underline{11} \frac{3}{2}\right)$ Hamiltonian.

- Let $\delta=0, \gamma=0$. On the Hamiltonian level, $\eta=0$. The Hamiltonian becomes

$$
\begin{aligned}
t H= & (\lambda-1)^{2} \lambda \mu^{2}-\left(\kappa_{0}(\lambda-1)^{2}+\left(\chi_{1}-1\right) \lambda(\lambda-1)\right) \mu \\
& +\frac{\left(\left(\kappa_{0}+\chi_{1}-1\right)^{2}-\kappa_{\infty}^{2}\right)(\lambda-1)}{4} .
\end{aligned}
$$

This case is solvable in quadratures by the method of Section B.3.

Note that the corresponding 2 nd order equation does not depend on the parameter $\chi_{1}$. On the Hamiltonian level it can be seen by using the canonical transformation $\tilde{\mu}=\mu-\frac{\chi_{1}-1}{2(\lambda-1)}$, which transforms (5.4) into

$$
t H=(\lambda-1)^{2} \lambda \tilde{\mu}^{2}-\kappa_{0}(\lambda-1)^{2} \tilde{\mu}+\frac{\left(\kappa_{0}^{2}-\kappa_{\infty}^{2}\right)(\lambda-1)}{4}-\frac{\left(\chi_{1}-1\right)^{2}}{4},
$$

where the dependence on $\chi_{1}$ remains only in the free term. 
Remark 5.1. It is well known that the Painlevé deg-V or $\left(\underline{11} \frac{3}{2}\right)$ and ndeg-III' or (22) equations are equivalent [19]. Below we will show this by describing a canonical transformation that connects the corresponding Hamiltonians.

Let us insert the canonical transformation

$$
\tilde{\lambda}=1-\frac{1}{\mu}, \quad \mu=\frac{1}{1-\tilde{\lambda}}, \quad \tilde{\mu}=\mu^{2} \lambda-\frac{\left(\chi_{0}-1\right) \mu}{2}, \quad \lambda=(1-\tilde{\lambda})^{2} \tilde{\mu}+\frac{\left(\chi_{0}-1\right)}{2}(1-\tilde{\lambda}),
$$

into the (22) Hamiltonian (5.6). We obtain

$$
t H=\tilde{\lambda}(\tilde{\lambda}-1)^{2} \tilde{\mu}^{2}+\frac{-\chi_{0}+\chi_{\infty}+1}{2}(\tilde{\lambda}-1)^{2} \tilde{\mu}-\frac{\chi_{\infty}\left(\chi_{0}-1\right)}{2}(\tilde{\lambda}-1)-\frac{t \tilde{\lambda}}{(\tilde{\lambda}-1)}-\frac{\left(\chi_{0}-1\right)^{2}}{4}+t,
$$

which after appropriate identification of parameters coincides with the $\left(\underline{11} \frac{3}{2}\right)$ Hamiltonian (5.3) up to a free term.

\subsection{Painlevé III' or (22)}

As noted in [19], the usual Painlevé III' equation, depending on 4 parameters, should be treated as a supertype. It is invariant with respect to two distinct scaling transformations. It includes 3 nontrivial types: nondegenerate III' depending on 2 parameters, degenerate III' (in two forms) depending on 1 parameter, doubly degenerate III' $^{\prime}$ with no parameters. There are also trivial forms solvable in quadratures.

In this subsection we discuss Painlevé III' in detail. We prefer to denote it by the symbol (22), because it corresponds to the supertype (22) of the Heun class.

$\diamond$ Painlevé III' or $(\underline{22})$ equation and Hamiltonian:

$$
\begin{aligned}
& \frac{\mathrm{d}^{2} \lambda}{\mathrm{d} t^{2}}=\frac{1}{\lambda}\left(\frac{\mathrm{d} \lambda}{\mathrm{d} t}\right)^{2}-\frac{1}{t} \frac{\mathrm{d} \lambda}{\mathrm{d} t}+\frac{\alpha \lambda^{2}+\gamma \lambda^{3}}{4 t^{2}}+\frac{\beta}{4 t}+\frac{\delta}{4 \lambda}, \\
& t H=\lambda^{2} \mu^{2}-\left(\eta_{\infty} \lambda^{2}+\left(\chi_{0}-1\right) \lambda-\eta_{0} t\right) \mu+\frac{1}{2} \eta_{\infty}\left(\chi_{0}+\chi_{\infty}-1\right) \lambda,
\end{aligned}
$$

where $\alpha=-4 \eta_{\infty} \chi_{\infty}, \beta=4 \eta_{0} \chi_{0}, \gamma=4 \eta_{\infty}^{2}, \delta=-4 \eta_{0}^{2}$. Scaling properties

$$
\begin{aligned}
& \frac{\epsilon^{2}}{\omega} P_{\alpha, \beta, \gamma, \delta}(\epsilon t, \omega \lambda)=P_{\omega \alpha, \frac{\epsilon}{\omega} \beta, \omega^{2} \gamma, \frac{\epsilon^{2}}{\omega^{2}} \delta}(t, \lambda), \\
& \epsilon H_{\eta_{0}, \eta_{\infty}, \chi_{0}, \chi_{\infty}}\left(\epsilon t, \omega \lambda, \frac{\mu}{\omega}\right)=H_{\frac{\epsilon}{\omega} \eta_{0}, \omega \eta_{\infty}, \chi_{0}, \chi_{\infty}}(t, \lambda, \mu) .
\end{aligned}
$$

* Painlevé ndeg-III' or (22) equation and Hamiltonian, recalled from (4.29) and (4.30):

$$
\begin{aligned}
& \frac{\mathrm{d}^{2} \lambda}{\mathrm{d} t^{2}}=\frac{1}{\lambda}\left(\frac{\mathrm{d} \lambda}{\mathrm{d} t}\right)^{2}-\frac{1}{t} \frac{\mathrm{d} \lambda}{\mathrm{d} t}+\frac{\alpha \lambda^{2}}{4 t^{2}}+\frac{\lambda^{3}}{t^{2}}+\frac{\beta}{4 t}-\frac{1}{\lambda}, \\
& t H:=\lambda^{2} \mu^{2}-\left(\lambda^{2}+\left(\chi_{0}-1\right) \lambda-t\right) \mu+\frac{1}{2}\left(\chi_{0}+\chi_{\infty}-1\right) \lambda,
\end{aligned}
$$

where $\alpha=-4 \chi_{\infty}, \beta=4 \chi_{0}$.

* Painlevé deg-III'-1 or $\left(2 ; \frac{3}{2}\right)$ equation and Hamiltonian, recalled from (4.32) and (4.31):

$$
\begin{aligned}
& \frac{\mathrm{d}^{2} \lambda}{\mathrm{d} t^{2}}=\frac{1}{\lambda}\left(\frac{\mathrm{d} \lambda}{\mathrm{d} t}\right)^{2}-\frac{1}{t} \frac{\mathrm{d} \lambda}{\mathrm{d} t}-\frac{\lambda^{2}}{t^{2}}+\frac{\beta}{4 t}-\frac{1}{\lambda}, \\
& t H=\lambda^{2} \mu^{2}+\left(\left(1-\chi_{0}\right) \lambda+t\right) \mu+\frac{\lambda}{2},
\end{aligned}
$$

where $\beta=4 \chi_{0}$. 
* Painlevé deg-III'-2 or $\left(\frac{3}{2} ; 2\right)$ equation and Hamiltonian recalled from (4.34) and (4.33):

$$
\begin{aligned}
& \frac{\mathrm{d}^{2} \lambda}{\mathrm{d} t^{2}}=\frac{1}{\lambda}\left(\frac{\mathrm{d} \lambda}{\mathrm{d} t}\right)^{2}-\frac{1}{t} \frac{\mathrm{d} \lambda}{\mathrm{d} t}+\frac{\alpha \lambda^{2}}{4 t^{2}}+\frac{\lambda^{3}}{t^{2}}+\frac{1}{t}, \\
& t H=\lambda^{2} \mu^{2}-\lambda^{2} \mu+\frac{\chi_{\infty} \lambda}{2}+\frac{t}{2 \lambda}
\end{aligned}
$$

where $\alpha=-4 \chi_{\infty}$.

* Painlevé ddeg-III' or $\left(\frac{3}{2} \frac{3}{2}\right)$ equation and Hamiltonian, recalled from (4.36) and (4.35):

$$
\begin{aligned}
& \frac{\mathrm{d}^{2} \lambda}{\mathrm{d} t^{2}}=\frac{1}{\lambda}\left(\frac{\mathrm{d} \lambda}{\mathrm{d} t}\right)^{2}-\frac{1}{t} \frac{\mathrm{d} \lambda}{\mathrm{d} t}-\frac{\lambda^{2}}{t^{2}}+\frac{1}{t}, \\
& t H=\lambda^{2} \mu^{2}+\lambda \mu+\frac{\lambda}{2}+\frac{t}{2 \lambda} .
\end{aligned}
$$

Let us discuss special cases:

- Let $\delta \neq 0, \gamma \neq 0$. On the level of the Hamiltonian it means $\eta_{0} \neq 0, \eta_{\infty} \neq 0$. By scaling we can set on the Hamiltonian level $\eta_{0}=1, \eta_{\infty}=1$, which corresponds to $\gamma=4, \delta=-4$. We obtain the Painlevé ndeg-III' or (22) equation (4.30) and Hamiltonian (4.29).

- Let $\delta, \alpha \neq 0, \gamma=0$. By scaling we can make $\delta=-4, \alpha=-4$. We obtain the Painlevé $\left(2 ; \frac{3}{2}\right)$ equation. This reduction does not work for the Painlevé $\left(2 ; \frac{3}{2}\right)$ Hamiltonian.

- Let $\delta=0, \gamma, \beta \neq 0$. By scaling we can make $\gamma=4, \beta=4$. We obtain the Painlevé $\left(\frac{3}{2} ; 2\right)$ equation. This reduction does not work for the Painlevé $\left(\frac{3}{2} ; 2\right)$ Hamiltonian.

The equations $\left(\frac{3}{2} ; 2\right)$ and $\left(2 ; \frac{3}{2}\right)$ are two equivalent forms of $\left(2 \frac{3}{2}\right)$ see Proposition 5.2.

- Let $\delta=\gamma=0, \alpha, \beta \neq 0$. By scaling we can make $\alpha=-4, \beta=4$. We obtain the Painlevé $\left(\frac{3}{2} \frac{3}{2}\right)$ equation (4.36). This reduction does not work for the Painlevé $\left(\frac{3}{2} \frac{3}{2}\right)$ Hamiltonian (4.35).

- Let $\alpha=\gamma=0$. On the Hamiltonian level, $\eta_{\infty}=0$. The Hamiltonian is

$$
t H=\lambda^{2} \mu^{2}-\left(\left(\chi_{0}-1\right) \lambda-\eta_{0} t\right) \mu .
$$

We can apply to (5.7) the time-dependent canonical transformation

$$
\tilde{H}=H-\frac{\tilde{\lambda} \tilde{\mu}}{t}, \quad \tilde{\lambda}=\frac{\lambda}{t}, \quad \tilde{\mu}=t \mu
$$

obtaining

$$
t \tilde{H}=\tilde{\lambda}^{2} \tilde{\mu}^{2}-\left(\chi_{0} \tilde{\lambda}-\eta_{0}\right) \tilde{\mu} .
$$

It is solvable by quadratures by Section B.3.

- Let $\beta=\delta=0$. On the Hamiltonian level it corresponds to $\eta_{0}=0$. The Hamiltonian is

$$
t H=\lambda^{2} \mu^{2}-\left(\eta_{\infty} \lambda^{2}+\left(\chi_{0}-1\right) \lambda\right) \mu+\frac{1}{2} \eta_{\infty}\left(\chi_{0}+\chi_{\infty}-1\right) \lambda .
$$

It is solvable by quadratures by Section B.3.

Proposition 5.2. The Painlevé $\left(2 ; \frac{3}{2}\right)$ and $\left(\frac{3}{2} ; 2\right)$ equations are equivalent. 
Proof. First we apply to the Painlevé $\left(2 ; \frac{3}{2}\right)$ Hamiltonian the time-dependent canonical transformation (5.8) obtaining

$$
t \tilde{H}=\tilde{\lambda}^{2} \tilde{\mu}^{2}+\left(-\chi_{0} \tilde{\lambda}+1\right) \tilde{\mu}+\frac{t \tilde{\lambda}}{2} .
$$

Next we apply the time independent canonical transformation

$$
\tilde{\lambda}=\frac{1}{\lambda}, \quad \tilde{\mu}=-\mu \lambda^{2}+\frac{\chi_{0} \lambda}{2},
$$

obtaining

$$
t \tilde{H}=\lambda^{2} \mu^{2}-\lambda^{2} \mu+\frac{\chi_{0} \lambda}{2}+\frac{t}{2 \lambda}-\frac{\chi_{0}^{2}}{4},
$$

which is the Painlevé $\left(\frac{3}{2} ; 2\right)$ Hamiltonian for $\chi_{\infty}=\chi_{0}$ minus $\frac{\chi_{0}^{2}}{4}$.

Remark 5.3. The standard Painlevé III equation is given by

$$
\frac{\mathrm{d}^{2} \tilde{\lambda}}{\mathrm{d} \tilde{t}^{2}}=\frac{1}{\lambda}\left(\frac{\mathrm{d} \tilde{\lambda}}{\mathrm{d} \tilde{t}}\right)^{2}-\frac{1}{\tilde{t}} \frac{\mathrm{d} \tilde{\lambda}}{\mathrm{d} \tilde{t}}+\frac{\alpha \tilde{\lambda}^{2}+\beta}{\tilde{t}}+\gamma \tilde{\lambda}^{3}+\frac{\delta}{\tilde{\lambda}} .
$$

The Painlevé III' equation is obtained from (5.9) by $\tilde{\lambda}=t \lambda, \tilde{t}=t^{2}$.

\subsection{Painlevé IV-34 or ( $\underline{13})$}

It has been noted in [19] that it is natural to consider Painlevé IV together with the so-called Painlevé 34. The latter is equivalent to Painlevé II, and therefore is not so well known. Together they can be treated as special cases of a supertype, which in [19] is denoted 4_34, and we denote by IV-34, or preferably by $(\underline{13})$, since it is related to the supertype ( $\underline{13})$ of the Heun class. In this subsection we discuss this supertype of Painlevé in detail.

Painlevé IV-34 depends on 3 parameters. It is invariant with respect to a scaling transformation. It contains Painlevé IV depending on 2 parameters and Painlevé 34 depending on 1 parameter, as well as a trivial type solvable in quadratures.

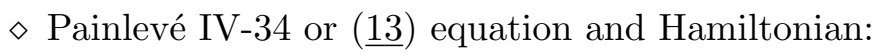

$$
\begin{aligned}
& \frac{\mathrm{d}^{2} \lambda}{\mathrm{d} t^{2}}=\frac{1}{2 \lambda}\left(\frac{\mathrm{d} \lambda}{\mathrm{d} t}\right)^{2}+\rho \lambda(2 \lambda+t)+\gamma \lambda(\lambda+t)(3 \lambda+t)+\frac{\beta}{4 \lambda}, \\
& H=\lambda \mu^{2}-\left(\eta \lambda^{2}+\eta t \lambda-\theta \lambda+\kappa_{0}\right) \mu+\left(\frac{\theta^{2}}{4}+\frac{\left(\kappa_{0}-1\right) \eta}{2}\right) \lambda,
\end{aligned}
$$

where $\beta=-2 \kappa_{0}^{2}, \rho=-\eta \theta, \gamma=\frac{1}{2} \eta^{2}$. Scaling properties

$$
\begin{aligned}
& \epsilon P_{\beta, \rho, \gamma}(\epsilon t, \epsilon \lambda)=P_{\beta, \epsilon^{3} \rho, \epsilon^{4} \gamma}(t, \lambda), \\
& \epsilon H_{\eta, \theta, \kappa_{0}}\left(\epsilon t, \epsilon \lambda, \epsilon^{-1} \mu\right)=H_{\epsilon^{2} \eta, \epsilon \theta, \kappa_{0}}(t, \lambda, \mu) .
\end{aligned}
$$

* Painlevé IV or (13) equation and Hamiltonian, recalled from (4.38) and (4.37):

$$
\begin{aligned}
& \frac{\mathrm{d}^{2} \lambda}{\mathrm{d} t^{2}}=\frac{1}{2 \lambda}\left(\frac{\mathrm{d} \lambda}{\mathrm{d} t}\right)^{2}+\frac{3}{2} \lambda^{3}+4 t \lambda^{2}+2\left(t^{2}-\alpha\right) \lambda+\frac{\beta}{\lambda}, \\
& H=2 \lambda \mu^{2}-\left(\lambda^{2}+2 t \lambda+2 \kappa_{0}\right) \mu+\theta_{\infty} \lambda,
\end{aligned}
$$

where $\alpha=-\kappa_{0}+2 \theta_{\infty}-1, \beta=-2 \kappa_{0}^{2}$. 
* Painlevé 34 or $\left(\underline{1} \frac{5}{2}\right)$ equation and Hamiltonian, recalled from (4.40) and (4.39):

$$
\begin{aligned}
& \frac{\mathrm{d}^{2} \lambda}{\mathrm{d} t^{2}}=\frac{1}{2 \lambda}\left(\frac{\mathrm{d} \lambda}{\mathrm{d} t}\right)^{2}+2 \lambda^{2}+t \lambda+\frac{\beta}{4 \lambda}, \\
& H=\lambda \mu^{2}-\kappa_{0} \mu-\frac{\lambda^{2}}{2}-\frac{t \lambda}{2},
\end{aligned}
$$

where $\beta=-2 \kappa_{0}^{2}$.

Let us discuss special cases:

- Let $\gamma \neq 0$. By scaling we can set $\gamma=\frac{1}{8}$. We change the time $\tilde{t}=\frac{1}{2} t+\rho$. We obtain the Painlevé (13) equation with $\alpha=4 \rho^{2}$.

The equivalent reduction on the Hamiltonian level: For $\eta \neq 0$, by scaling, we can make $\eta=\frac{1}{2}$. We multiply the Hamiltonian by 2 and change $t$ to $2 t$ :

$$
2 H(2 t, \lambda, \mu)=2 \lambda \mu^{2}-\left(\lambda^{2}+2 t \lambda-2 \theta \lambda+\kappa_{0}\right) \mu+\frac{\theta^{2}+\kappa_{0}-1}{2} \lambda,
$$

which is the Painlevé (13) Hamiltonian with $\theta_{\infty}=\frac{\theta^{2}+\kappa_{0}-1}{2}$ and $\tilde{t}=t-\theta$.

- Let $\gamma=0, \rho \neq 0$. By scaling we can make $\rho=1$. We obtain the Painlevé 34 or $\left(\underline{1} \frac{5}{2}\right)$ equation. This reduction does not work for the Painlevé 34 or $\left(\underline{1} \frac{5}{2}\right)$ Hamiltonian.

- Let $\gamma=\rho=0$, which on the Hamiltonian level corresponds to $\eta=0$. We have the Hamiltonian

$$
H=\lambda \mu^{2}-\left(-\theta \lambda+\kappa_{0}\right) \mu+\frac{\theta^{2}}{4} \lambda
$$

which is solvable by quadratures (see Section B.3).

Remark 5.4. Note that Painlevé 34 or $\left(\underline{1} \frac{5}{2}\right)$ is equivalent to Painlevé II or (4).

Let us show this on the Hamiltonian level. After an application of the canonical transformation

$$
\tilde{\mu}=\lambda, \quad \tilde{\lambda}=-\mu,
$$

the Painlevé $\left(\underline{1} \frac{5}{2}\right)$ Hamiltonian (5.11) becomes

$$
H=-\frac{\tilde{\mu}^{2}}{2}-\tilde{\lambda}^{2} \tilde{\mu}-\frac{t \tilde{\mu}}{2}+\kappa_{0} \tilde{\lambda} .
$$

Then we change $t$ into $-t$ and multiply the Hamiltonian by -1 , obtaining the Painlevé (4) Hamiltonian (5.12) with $\kappa_{0}=\alpha+\frac{1}{2}$.

\subsection{Painlevé I-II or ( $\underline{4})$}

Usually Painlevé I and II equations are treated separately. However, it has been noted already by Painlevé and elaborated in [19] that it is natural to join them in a single supertype. [19] denotes it $1 \_2$, we denote it I-II, or preferably $(\underline{4})$, since it corresponds to the supertype ( $\left.\underline{4}\right)$ of the Heun class. In this subsection we discuss this supertype of Painlevé in detail.

Painlevé I-II depends on 2 parameters. It is invariant with respect to a scaling transformation. It contains Painlevé II, depending on 1 parameter, Painlevé I with no parameters and a trivial type solvable in quadratures. 
$\diamond$ Painlevé I-II or ( $\underline{4})$ equation and Hamiltonian:

$$
\begin{aligned}
& \frac{\mathrm{d}^{2} \lambda}{\mathrm{d} t^{2}}=\gamma\left(2 \lambda^{3}+t \lambda\right)+\beta\left(6 \lambda^{2}+t\right) \\
& H=\frac{1}{2} \mu^{2}-\left(\eta \lambda^{2}+\frac{1}{2} \eta t\right) \mu-2 \beta \lambda^{3}-t \beta \lambda-\frac{1}{2} \eta \lambda
\end{aligned}
$$

where $\gamma=\eta^{2}$.

Scaling properties:

$$
\begin{aligned}
& \epsilon^{3} P_{\gamma, \beta}\left(\epsilon^{2} t, \epsilon \lambda\right)=P_{\epsilon^{6} \gamma, \epsilon^{5} \beta}(t, \lambda), \\
& \epsilon^{2} H_{\eta, \beta}\left(\epsilon^{2} t, \epsilon \lambda, \epsilon^{-1} \mu\right)=H_{\epsilon^{3} \eta, \epsilon^{5} \beta}(t, \lambda, \mu) .
\end{aligned}
$$

* Painlevé II or (4) equation and Hamiltonian, recalled from (4.42) and (4.41):

$$
\begin{aligned}
& \frac{\mathrm{d}^{2} \lambda}{\mathrm{d} t^{2}}=2 \lambda^{3}+t \lambda+\alpha \\
& H=\frac{1}{2} \mu^{2}-\left(\lambda^{2}+\frac{t}{2}\right) \mu-\left(\alpha+\frac{1}{2}\right) \lambda .
\end{aligned}
$$

* Painlevé I or $\left(\frac{7}{2}\right)$ equation and Hamiltonian, recalled from (4.44) and (4.43):

$$
\begin{aligned}
& \frac{\mathrm{d}^{2} \lambda}{\mathrm{d} t^{2}}=6 \lambda^{2}+t \\
& H=\frac{1}{2} \mu^{2}-2 \lambda^{3}-t \lambda
\end{aligned}
$$

Let us discuss special cases:

- Let $\eta \neq 0$, in both the equation and the Hamiltonian. By scaling we can set $\eta=1$. We apply the canonical transformation

$$
t=\tilde{t}+6 \beta^{2}, \quad \lambda=\tilde{\lambda}-\beta, \quad \mu=\tilde{\mu}-2 \beta \tilde{\lambda}+4 \beta^{2} .
$$

We obtain

$$
H=\frac{\tilde{\mu}^{2}}{2}-\left(\tilde{\lambda}^{2}+\frac{\tilde{t}}{2}\right) \mu-\left(2 \beta^{3}+\frac{1}{2}\right) \tilde{\lambda}+\frac{1}{2} \beta-\beta^{2} \tilde{t} .
$$

Thus up to free terms we obtain the Painlevé (4) Hamiltonian with $\alpha=2 \beta^{3}$, and hence also the Painlevé (4) equation.

- Let $\eta=0, \beta \neq 0$. By scaling we can set $\beta=1$. The Hamiltonian becomes

$$
H=\frac{\mu^{2}}{2}-2 \lambda^{3}-t \lambda
$$

Thus we obtain the Painlevé $\left(\frac{7}{2}\right)$ Hamiltonian, and hence also the Painlevé $\left(\frac{7}{2}\right)$ equation.

- Let $\eta=0, \beta=0$. The Hamiltonian becomes $H=\frac{1}{2} \mu^{2}$, which is trivial. 


\section{A Proof of Theorems 4.1, 4.2 and 4.3}

\section{A.1 Preparation for the proof of Theorem 4.1}

Recall from (4.7), (4.8) and (4.12) that we set

$$
\begin{aligned}
& p(z):=p_{0}(z)-\frac{1}{z-\lambda}, \quad p_{0}(z):=\frac{\tau(z)}{\sigma(z)}, \\
& q(z):=q_{0}(z)+\frac{1}{\sigma(z)}\left(-\eta(\lambda)-\mu\left(\tau(\lambda)-\sigma^{\prime}(\lambda)\right)-\mu^{2} \sigma(\lambda)+\frac{\mu \sigma(\lambda)}{(z-\lambda)}\right), \quad q_{0}(z):=\frac{\eta(z)}{\sigma(z)}, \\
& a(z):=\frac{c(z)}{z-\lambda}, \quad b(z):=-\frac{c(\lambda) \mu}{z-\lambda}
\end{aligned}
$$

where $c(z)$ is a $t, \lambda$-dependent polynomial of degree $\leq 2$. Recall that the prime is synonymous with $\partial_{z}$ and the dot with $\partial_{t}$.

We will find conditions on $c$ and the time variable $t$ so that the compatibility conditions (4.5) and (4.6), that is,

$$
\begin{aligned}
& \dot{p}-a p^{\prime}+2 b^{\prime}-p a^{\prime}+a^{\prime \prime}=0, \\
& \dot{q}+p b^{\prime}-2 q a^{\prime}-a q^{\prime}+b^{\prime \prime}=0,
\end{aligned}
$$

are satisfied.

The following simple identities will be useful in our calculations:

\section{Lemma A.1.}

$$
\begin{aligned}
& \frac{(\lambda-s)}{(z-\lambda)}\left(\frac{1}{z-s}-\frac{1}{\lambda-s}+\frac{z-\lambda}{(\lambda-s)^{2}}\right)=\frac{1}{\lambda-s}-\frac{1}{z-s} \\
& \frac{2}{z-s}-\frac{2}{\lambda-s}+(z-\lambda)\left(\frac{1}{(z-s)^{2}}+\frac{1}{(\lambda-s)^{2}}\right)=\frac{(z-\lambda)^{3}}{(z-s)^{2}(\lambda-s)^{2}} .
\end{aligned}
$$

Let $\psi:=\frac{\xi(z)}{z-s}$. If $\xi$ be a polynomial with $\operatorname{deg} \xi \leq 2$, then

$$
\begin{aligned}
& \xi(z)-\xi(\lambda)-(z-\lambda) \xi^{\prime}(z)=-\frac{\xi^{\prime \prime}}{2}(z-\lambda)^{2}, \\
& \psi(z)-\psi(\lambda)-(z-\lambda) \psi^{\prime}(z)=-\frac{\xi(s)(z-\lambda)^{2}}{(z-s)^{2}(\lambda-s)} .
\end{aligned}
$$

If $\xi$ is a polynomial with $\operatorname{deg} \xi \leq 3$, then

$$
\begin{aligned}
& 2 \xi(z)-2 \xi(\lambda)-(z-\lambda)\left(\xi^{\prime}(z)+\xi^{\prime}(\lambda)\right)=-\frac{\xi^{\prime \prime \prime}}{6}(z-\lambda)^{3} \\
& 2 \psi(z)-2 \psi(\lambda)-(z-\lambda)\left(\psi^{\prime}(z)+\psi^{\prime}(\lambda)\right)=\xi(s) \frac{(z-\lambda)^{3}}{(z-s)^{2}(\lambda-s)^{2}} .
\end{aligned}
$$

\section{A.2 First compatibility condition}

Proposition A.2. Suppose that the following equation of motion for $\lambda$ holds:

$$
\dot{\lambda}=2 c(\lambda) \mu-c^{\prime}(\lambda)+\frac{c \tau}{\sigma}(\lambda),
$$

and we have the condition

$$
0=I:=\frac{\dot{\tau}}{\sigma}(z)-\frac{\tau \dot{\sigma}}{\sigma^{2}}(z)+\frac{\frac{c \tau}{\sigma}(z)-\frac{c \tau}{\sigma}(\lambda)-(z-\lambda)\left(\frac{c \tau}{\sigma}\right)^{\prime}(z)}{(z-\lambda)^{2}} .
$$

Then (A.1) is satisfied. 


\section{Proof.}

$$
\begin{aligned}
0= & \dot{p}(z)-a(z) p^{\prime}(z)+2 b^{\prime}(z)-p(z) a^{\prime}(z)+a^{\prime \prime}(z) \\
= & \dot{p}_{0}(z)-\frac{\dot{\lambda}}{(z-\lambda)^{2}}-\frac{c(z)}{z-\lambda}\left(p_{0}^{\prime}(z)+\frac{1}{(z-\lambda)^{2}}\right)+\frac{2 c(\lambda) \mu}{(z-\lambda)^{2}} \\
& -\left(p_{0}(z)-\frac{1}{(z-\lambda)}\right)\left(\frac{c^{\prime}(z)}{z-\lambda}-\frac{c(z)}{(z-\lambda)^{2}}\right)+\frac{c^{\prime \prime}(z)}{z-\lambda}-\frac{2 c^{\prime}(z)}{(z-\lambda)^{2}}+\frac{2 c(z)}{(z-\lambda)^{3}} \\
= & \frac{1}{(z-\lambda)^{2}}\left(-\dot{\lambda}+2 c(\lambda) \mu-c^{\prime}(z)+\left(c p_{0}\right)(z)\right)+\frac{1}{(z-\lambda)}\left(-c(z) p_{0}^{\prime}(z)-p_{0}(z) c^{\prime}(z)+c^{\prime \prime}(z)\right) \\
& +\dot{p}_{0}(z) .
\end{aligned}
$$

We rearrange this as

$$
\begin{aligned}
= & \frac{1}{(z-\lambda)^{2}}\left(-\dot{\lambda}+2 c(\lambda) \mu-c^{\prime}(\lambda)+\left(c p_{0}\right)(\lambda)\right) \\
& +\frac{-c^{\prime}(z)+c^{\prime}(\lambda)+c^{\prime \prime}(z)(z-\lambda)}{(z-\lambda)^{2}} \\
& +\frac{\left(c p_{0}\right)(z)-\left(c p_{0}\right)(\lambda)-(z-\lambda)\left(c p_{0}\right)^{\prime}(z)}{(z-\lambda)^{2}}+\dot{p}_{0}(z) .
\end{aligned}
$$

(A.9) is proportional to $\frac{1}{(z-\lambda)^{2}}$ and the last two lines are regular at $z=\lambda$. Therefore, (A.9) has to vanish separately, yielding the condition (A.7). (A.10) vanishes automatically, because $c$ is a polynomial of degree $\leq 2$ in $z$. (A.11) yields the condition (A.8).

\section{A.3 Second compatibility condition}

It is much more difficult to analyze the second compatibility condition.

Proposition A.3. Suppose that the equation for $\lambda$ (A.7) holds together with the equation for $\mu$

$$
\dot{\mu}=-\frac{c \eta^{\prime}}{\sigma}(\lambda)-\mu\left(\frac{c \tau^{\prime}}{\sigma}(\lambda)-\frac{c \sigma^{\prime \prime}}{2 \sigma}(\lambda)-\frac{c^{\prime \prime}}{2}\right)-\mu^{2} \frac{\sigma^{\prime} c}{\sigma}(\lambda)
$$

Assume also that $I=0$ (see (A.8)) and the following conditions are satisfied:

$$
\begin{aligned}
0=I I:= & -\frac{\dot{\sigma}}{\sigma}(z)(\eta(z)-\eta(\lambda))+\dot{\eta}(z)-\dot{\eta}(\lambda) \\
& +\frac{\eta(z)-\eta(\lambda)}{(z-\lambda)}\left(\frac{c \sigma^{\prime}}{\sigma}(z)-c^{\prime}(z)\right)-\eta^{\prime}(\lambda)\left(\frac{\sigma^{\prime} c}{\sigma}(\lambda)-c^{\prime}(\lambda)\right) \\
& +\frac{2 c \eta(z)-2 c \eta(\lambda)-\left((c \eta)^{\prime}(z)+(c \eta)^{\prime}(\lambda)\right)(z-\lambda)}{(z-\lambda)^{2}} ; \\
0=I I I:= & \frac{\dot{\sigma}}{\sigma}(z)-\frac{\dot{\sigma}}{\sigma}(\lambda)-\frac{\frac{c \sigma^{\prime}}{\sigma}(z)-\frac{c \sigma^{\prime}}{\sigma}(\lambda)-\left(\frac{c \sigma^{\prime}}{\sigma}\right)^{\prime}(\lambda)(z-\lambda)}{(z-\lambda)} .
\end{aligned}
$$

Then (A.2) is true.

\section{Proof.}

$$
\begin{aligned}
0 & =\dot{q}(z)+p(z) b^{\prime}(z)-2 q(z) a^{\prime}(z)-a(z) q^{\prime}(z)+b^{\prime \prime}(z) \\
& =\frac{1}{\sigma(z)}\left(-\tau(\lambda)+\sigma^{\prime}(\lambda)-2 \mu \sigma(\lambda)+\frac{\sigma(\lambda)}{(z-\lambda)}\right) \dot{\mu}
\end{aligned}
$$




$$
\begin{aligned}
& +\frac{1}{\sigma(z)}\left(-\eta^{\prime}(\lambda)-\mu\left(\tau^{\prime}(\lambda)-\sigma^{\prime \prime}(\lambda)\right)-\mu^{2} \sigma^{\prime}(\lambda)+\frac{\mu \sigma^{\prime}(\lambda)}{(z-\lambda)}+\frac{\mu \sigma(\lambda)}{(z-\lambda)^{2}}\right) \dot{\lambda} \\
& -\frac{\dot{\sigma}(z)}{\sigma^{2}(z)}\left(\eta(z)-\eta(\lambda)-\mu\left(\tau(\lambda)-\sigma^{\prime}(\lambda)\right)-\mu^{2} \sigma(\lambda)+\frac{\mu \sigma(\lambda)}{(z-\lambda)}\right) \\
& +\frac{1}{\sigma(z)}\left(\dot{\eta}(z)-\dot{\eta}(\lambda)-\mu\left(\dot{\tau}(\lambda)-\dot{\sigma}^{\prime}(\lambda)\right)-\mu^{2} \dot{\sigma}(\lambda)+\frac{\mu \dot{\sigma}(\lambda)}{(z-\lambda)}\right)+\left(\frac{\tau(z)}{\sigma(z)}-\frac{1}{(z-\lambda)}\right) \frac{c(\lambda) \mu}{(z-\lambda)^{2}} \\
& -\frac{2}{\sigma(z)}\left(\eta(z)-\eta(\lambda)-\mu\left(\tau(\lambda)-\sigma^{\prime}(\lambda)\right)-\mu^{2} \sigma(\lambda)+\frac{\mu \sigma(\lambda)}{(z-\lambda)}\right)\left(\frac{c^{\prime}(z)}{(z-\lambda)}-\frac{c(z)}{(z-\lambda)^{2}}\right) \\
& +\frac{c(z) \sigma^{\prime}(z)}{(z-\lambda) \sigma^{2}(z)}\left(\eta(z)-\eta(\lambda)-\mu\left(\tau(\lambda)-\sigma^{\prime}(\lambda)\right)-\mu^{2} \sigma(\lambda)+\frac{\mu \sigma(\lambda)}{(z-\lambda)}\right) \\
& -\frac{c(z)}{(z-\lambda) \sigma(z)}\left(\eta^{\prime}(z)-\frac{\mu \sigma(\lambda)}{(z-\lambda)^{2}}\right)-2 \frac{c(\lambda) \mu}{(z-\lambda)^{3}} .
\end{aligned}
$$

Next we collect the terms that contain an inverse power of $z-\lambda$. These terms are grouped in several categories. In these terms we also insert (A.7). We obtain

$$
\begin{aligned}
& =\frac{1}{\sigma(z)}\left(-\tau(\lambda)+\sigma^{\prime}(\lambda)-2 \mu \sigma(\lambda)\right) \dot{\mu}+\frac{1}{\sigma(z)}\left(-\eta^{\prime}(\lambda)-\mu\left(\tau^{\prime}(\lambda)-\sigma^{\prime \prime}(\lambda)\right)-\mu^{2} \sigma^{\prime}(\lambda)\right) \dot{\lambda} \\
& -\frac{\dot{\sigma}(z)}{\sigma^{2}(z)}\left(\eta(z)-\eta(\lambda)-\mu\left(\tau(\lambda)-\sigma^{\prime}(\lambda)\right)-\mu^{2} \sigma(\lambda)\right) \\
& +\frac{1}{\sigma(z)}\left(\dot{\eta}(z)-\dot{\eta}(\lambda)-\mu\left(\dot{\tau}(\lambda)-\dot{\sigma}^{\prime}(\lambda)\right)-\mu^{2} \dot{\sigma}(\lambda)\right) \\
& +\frac{1}{(z-\lambda) \sigma(z)}\left(-\mu \frac{\dot{\sigma}(z) \sigma(\lambda)}{\sigma(z)}+\mu \dot{\sigma}(\lambda)+\dot{\mu} \sigma(\lambda)\right)+\frac{1}{(z-\lambda)^{2} \sigma(z)} 2 c(z)(\eta(z)-\eta(\lambda)) \\
& +\frac{1}{(z-\lambda) \sigma(z)}\left(-2 c^{\prime}(z)(\eta(z)-\eta(\lambda))+c(z) \frac{\sigma^{\prime}(z)}{\sigma(z)}(\eta(z)-\eta(\lambda))-c(z) \eta^{\prime}(z)\right) \\
& +\frac{\mu}{(z-\lambda)^{2} \sigma(z)}(c(\lambda) \tau(\lambda)+c(\lambda) \tau(z)-2 c(z) \tau(\lambda)) \\
& +\frac{\mu}{(z-\lambda) \sigma(z)}\left(c(\lambda) \frac{\sigma^{\prime}(\lambda)}{\sigma(\lambda)} \tau(\lambda)-c(z) \frac{\sigma^{\prime}(z)}{\sigma(z)} \tau(\lambda)+2 c^{\prime}(z) \tau(\lambda)\right) \\
& +\frac{\mu}{(z-\lambda)^{3} \sigma(z)}(3 c(z) \sigma(\lambda)-3 c(\lambda) \sigma(z)) \\
& +\frac{\mu}{(z-\lambda)^{2} \sigma(z)}\left(-c^{\prime}(\lambda) \sigma(\lambda)-2 c^{\prime}(z) \sigma(\lambda)+2 c(z) \sigma^{\prime}(\lambda)+c(z) \frac{\sigma^{\prime}(z)}{\sigma(z)} \sigma(\lambda)\right) \\
& +\frac{\mu}{(z-\lambda) \sigma(z)}\left(-c^{\prime}(\lambda) \sigma^{\prime}(\lambda)-2 c^{\prime}(z) \sigma^{\prime}(\lambda)+c(z) \frac{\sigma^{\prime}(z)}{\sigma(z)} \sigma^{\prime}(\lambda)\right) \\
& +\frac{\mu^{2}}{(z-\lambda)^{2} \sigma(z)}(2 c(\lambda) \sigma(\lambda)-2 c(z) \sigma(\lambda)) \\
& +\frac{\mu^{2}}{(z-\lambda) \sigma(z)}\left(2 c(\lambda) \sigma^{\prime}(\lambda)+2 c^{\prime}(z) \sigma(\lambda)-c(z) \frac{\sigma^{\prime}(z)}{\sigma(z)} \sigma(\lambda)\right) \\
& =-\frac{1}{\sigma(z)}\left(\eta^{\prime}(\lambda)+\mu\left(\tau^{\prime}(\lambda)-\sigma^{\prime \prime}(\lambda)\right)+\mu^{2} \sigma^{\prime}(\lambda)\right) \dot{\lambda}-\frac{1}{\sigma(z)}\left(\tau(\lambda)-\sigma^{\prime}(\lambda)+2 \mu \sigma(\lambda)\right) \dot{\mu} \\
& +\frac{\sigma(\lambda)}{\sigma(z)(z-\lambda)} \dot{\mu}+\frac{1}{\sigma(z)}\left(-\frac{\dot{\sigma}}{\sigma}(z)(\eta(z)-\eta(\lambda))+\dot{\eta}(z)-\dot{\eta}(\lambda)\right) \\
& +\frac{\mu}{\sigma(z)}\left((\lambda) \frac{\dot{\sigma}}{\sigma}(z)-\dot{\tau}(\lambda)\right)+\frac{\mu}{\sigma(z)}\left(-\sigma^{\prime}(\lambda) \frac{\dot{\sigma}}{\sigma}(z)+\dot{\sigma}^{\prime}(\lambda)-\sigma(\lambda) \frac{\left(\frac{\dot{\sigma}}{\sigma}(z)-\frac{\dot{\sigma}}{\sigma}(\lambda)\right)}{(z-\lambda)}\right) \\
& +\frac{\mu^{2}}{\sigma(z)} \sigma(\lambda)\left(\frac{\dot{\sigma}}{\sigma}(z)-\frac{\dot{\sigma}}{\sigma}(\lambda)\right)+\frac{1}{(z-\lambda) \sigma(z)} c(\lambda) \eta^{\prime}(\lambda)
\end{aligned}
$$




$$
\begin{aligned}
& +\frac{1}{\sigma(z)}\left(\frac{2 c \eta(z)-2 c \eta(\lambda)-\left((c \eta)^{\prime}(z)+(c \eta)^{\prime}(\lambda)\right)(z-\lambda)}{(z-\lambda)^{2}}+\frac{\eta(z)-\eta(\lambda)}{(z-\lambda)}\left(c \frac{\sigma^{\prime}}{\sigma}(z)-c^{\prime}(z)\right)\right) \\
& +\frac{\mu}{\sigma(z)(z-\lambda)} c(\lambda) \tau^{\prime}(\lambda)+\frac{\mu}{\sigma(z)}\left(\frac{\left(\frac{\sigma^{\prime} c}{\sigma}(\lambda)-\frac{\sigma^{\prime} c}{\sigma}(z)\right)}{(z-\lambda)} \tau(\lambda)+c(\lambda) \frac{\tau^{\prime \prime}}{2}+c^{\prime \prime} \tau(\lambda)\right) \\
& +\frac{\mu}{(z-\lambda) \sigma(z)}\left(-\frac{c^{\prime \prime} \sigma(\lambda)}{2}-\frac{c(\lambda) \sigma^{\prime \prime}(\lambda)}{2}\right) \\
& +\frac{\mu}{\sigma(z)}\left(-c^{\prime \prime} \sigma^{\prime}(\lambda)+\frac{\left(\frac{c \sigma^{\prime}}{\sigma}(z)-\frac{c \sigma^{\prime}}{\sigma}(\lambda)-(z-\lambda)\left(\frac{c \sigma^{\prime}}{\sigma}\right)^{\prime}(\lambda)\right)}{(z-\lambda)^{2}} \sigma(\lambda)\right. \\
& \left.\quad+\frac{\left(\frac{c \sigma^{\prime}}{\sigma}(z)-\frac{c \sigma^{\prime}}{\sigma}(\lambda)\right)}{z-\lambda} \sigma^{\prime}(\lambda)-\frac{\sigma^{\prime \prime \prime} c(\lambda)}{2}\right) \\
& +\frac{\mu^{2}}{\sigma(z)(z-\lambda)} c(\lambda) \sigma^{\prime}(\lambda)+\frac{\mu^{2}}{\sigma(z)}\left(c^{\prime \prime} \sigma(\lambda)-\sigma(\lambda) \frac{\left(\frac{\sigma^{\prime} c}{\sigma}(z)-\frac{\sigma^{\prime} c}{\sigma}(\lambda)\right)}{(z-\lambda)}\right) .
\end{aligned}
$$

The singular term equals

$$
\frac{1}{\sigma(z)(z-\lambda)}\left(\sigma(\lambda) \dot{\mu}+c(\lambda) \eta^{\prime}(\lambda)+\mu\left(c(\lambda) \tau^{\prime}(\lambda)-\frac{1}{2} c(\lambda) \sigma^{\prime \prime}(\lambda)-\frac{1}{2} c^{\prime \prime} \sigma(\lambda)\right)+\mu^{2} c(\lambda) \sigma^{\prime}(\lambda)\right) .
$$

It yields the equation for $\dot{\mu}$, that is (A.12). After inserting (A.7) and (A.12) the first two lines become

$$
\begin{aligned}
& \frac{1}{\sigma(z) \sigma(\lambda)} \eta^{\prime}(\lambda)\left(-c(\lambda) \sigma^{\prime}(\lambda)+c^{\prime}(\lambda) \sigma(\lambda)\right) \\
& \quad+\frac{\mu}{\sigma(z) \sigma(\lambda)}\left(\tau^{\prime}(\lambda)\left(-c(\lambda) \sigma^{\prime}(\lambda)+c^{\prime}(\lambda) \sigma(\lambda)\right)+\tau(\lambda)\left(-\frac{c^{\prime \prime} \sigma(\lambda)}{2}+\frac{c(\lambda) \sigma^{\prime \prime}(\lambda)}{2}\right)\right) \\
& \quad+\frac{\mu}{\sigma(z) \sigma(\lambda)}\left(\frac{c^{\prime \prime} \sigma^{\prime}(\lambda) \sigma(\lambda)}{2}+\frac{c(\lambda) \sigma^{\prime}(\lambda) \sigma^{\prime \prime}(\lambda)}{2}-c^{\prime}(\lambda) \sigma(\lambda) \sigma^{\prime \prime}(\lambda)\right) \\
& \quad+\frac{\mu^{2}}{\sigma(z) \sigma(\lambda)}\left(-c(\lambda) \sigma^{\prime}(\lambda)^{2}+c^{\prime}(\lambda) \sigma(\lambda) \sigma^{\prime}(\lambda)-c^{\prime \prime} \sigma(\lambda)^{2}+c(\lambda) \sigma^{\prime \prime}(\lambda) \sigma(\lambda)\right) .
\end{aligned}
$$

Finally, we obtain

$$
\begin{aligned}
& =\frac{1}{\sigma(z)}\left(-\frac{\dot{\sigma}}{\sigma}(z)(\eta(z)-\eta(\lambda))+\dot{\eta}(z)-\dot{\eta}(\lambda)+\frac{\eta(z)-\eta(\lambda)}{(z-\lambda)}\left(\frac{c \sigma^{\prime}}{\sigma}(z)-c^{\prime}(z)\right)\right. \\
& \left.-\eta^{\prime}(\lambda)\left(\frac{\sigma^{\prime} c}{\sigma}(\lambda)-c^{\prime}(\lambda)\right)+\frac{2 c \eta(z)-2 c \eta(\lambda)-\left((c \eta)^{\prime}(z)+(c \eta)^{\prime}(\lambda)\right)(z-\lambda)}{(z-\lambda)^{2}}\right) \\
& +\frac{\mu}{\sigma(z)}\left(-\dot{\tau}(\lambda)+c(\lambda) \frac{\tau^{\prime \prime}}{2}-\tau^{\prime}(\lambda)\left(\frac{\sigma^{\prime} c}{\sigma}(\lambda)-c^{\prime}(\lambda)\right)\right. \\
& \left.+\tau(\lambda)\left(\frac{\dot{\sigma}}{\sigma}(z)+\frac{c^{\prime \prime}}{2}+\frac{c(\lambda) \sigma^{\prime \prime}(\lambda)}{2 \sigma(\lambda)}-\frac{\left(\frac{\sigma^{\prime} c}{\sigma}(z)-\frac{\sigma^{\prime} c}{\sigma}(\lambda)\right)}{(z-\lambda)}\right)\right) \\
& +\frac{\mu}{\sigma(z)}\left(-\sigma^{\prime}(\lambda) \frac{\dot{\sigma}}{\sigma}(z)+\dot{\sigma}^{\prime}(\lambda)-\sigma(\lambda) \frac{\left(\frac{\dot{\sigma}}{\sigma}(z)-\left(\frac{\dot{\sigma}}{\sigma}(\lambda)\right)\right.}{(z-\lambda)}\right. \\
& -\frac{c^{\prime \prime}}{2} \sigma^{\prime}(\lambda)+\frac{c(\lambda)}{2} \frac{\sigma^{\prime}(\lambda)}{\sigma(\lambda)} \sigma^{\prime \prime}(\lambda)-c^{\prime}(\lambda) \sigma^{\prime \prime}(\lambda)-\frac{\sigma^{\prime \prime \prime} c(\lambda)}{2} \\
& \left.+\frac{\left(\frac{c \sigma^{\prime}}{\sigma}(z)-\frac{c \sigma^{\prime}}{\sigma}(\lambda)-(z-\lambda)\left(\frac{c \sigma^{\prime}}{\sigma}\right)^{\prime}(\lambda)\right)}{(z-\lambda)^{2}} \sigma(\lambda)+\frac{\left(\frac{c \sigma^{\prime}}{\sigma}(z)-\frac{c \sigma^{\prime}}{\sigma}(\lambda)\right)}{z-\lambda} \sigma^{\prime}(\lambda)\right)
\end{aligned}
$$




$$
\begin{aligned}
& +\frac{\mu^{2} \sigma(\lambda)}{\sigma(z)}\left(\frac{\dot{\sigma}}{\sigma}(z)-\frac{\dot{\sigma}}{\sigma}(\lambda)-\frac{\frac{c \sigma^{\prime}}{\sigma}(z)-\frac{c \sigma^{\prime}}{\sigma}(\lambda)-\left(\frac{c \sigma^{\prime}}{\sigma}\right)^{\prime}(\lambda)(z-\lambda)}{(z-\lambda)}\right) \\
= & \frac{1}{\sigma(z)} I I+\left(-\frac{\mu \sigma(\lambda)}{\sigma(z)(z-\lambda)}+\frac{\mu}{\sigma(z)}\left(\tau(\lambda)-\sigma^{\prime}(\lambda)\right)+\frac{\mu^{2} \sigma(\lambda)}{\sigma(z)}\right) I I I \\
& +\frac{\mu}{\sigma(z)}\left(\left(\tau(\lambda)-\sigma^{\prime}(\lambda)\right) \frac{\dot{\sigma}}{\sigma}(\lambda)-\dot{\tau}(\lambda)+\dot{\sigma}^{\prime}(\lambda)\right) \\
& +\frac{\mu}{\sigma(z)}\left(\frac{1}{2}\left(\left(\tau-\sigma^{\prime}\right) c\right)^{\prime \prime}(\lambda)-\left(\tau-\sigma^{\prime}\right)^{\prime}(\lambda) \frac{c \sigma^{\prime}}{\sigma}(\lambda)\right. \\
& \left.\quad+\left(\tau-\sigma^{\prime}\right)(\lambda)\left(-\frac{c^{\prime} \sigma^{\prime}}{\sigma}(\lambda)+\frac{c \sigma^{\prime} \sigma^{\prime}}{\sigma^{2}}(\lambda)-\frac{c \sigma^{\prime \prime}}{2 \sigma}(\lambda)\right)\right) \\
= & \frac{1}{\sigma(z)} I I+\left(-\frac{\mu \sigma(\lambda)}{\sigma(z)(z-\lambda)}+\frac{\mu}{\sigma(z)}\left(\tau(\lambda)-\sigma^{\prime}(\lambda)\right)+\frac{\mu^{2} \sigma(\lambda)}{\sigma(z)}\right) I I I \\
& +\frac{\mu \sigma(\lambda)}{\sigma(z)}\left(-\partial_{t} \frac{\tau}{\sigma}(\lambda)+\partial_{t} \frac{\sigma^{\prime}}{\sigma}(\lambda)+\frac{1}{2}\left(\frac{\tau c}{\sigma}\right)^{\prime \prime}(\lambda)-\frac{1}{2}\left(\frac{\sigma^{\prime} c}{\sigma}\right)^{\prime \prime}(\lambda)\right) .
\end{aligned}
$$

We have

$$
\begin{aligned}
& \lim _{z \rightarrow \lambda} I=\partial_{t} \frac{\tau}{\sigma}(\lambda)-\frac{1}{2}\left(\frac{\tau c}{\sigma}\right)^{\prime \prime}(\lambda), \\
& \lim _{z \rightarrow \lambda} \frac{I I I}{(z-\lambda)}=\partial_{t} \frac{\sigma^{\prime}}{\sigma}(\lambda)-\frac{1}{2}\left(\frac{\sigma^{\prime} c}{\sigma}\right)^{\prime \prime}(\lambda) .
\end{aligned}
$$

Therefore, if $I$ and $I I I$ vanish, then so do (A.14) and (A.15), and hence also (A.13)

Propositions A.2 and A.3 prove Theorem 4.1.

Next we would like to prove Theorems 4.2 and 4.3. The proof will be divided into three subsections. In the first two we consider Cases A and in the third Case B.

From now on we assume that $\sigma, \tau, \eta$ correspond to a Heun class equation, that is $\operatorname{deg} \sigma \leq 3$, $\operatorname{deg} \tau \leq 2$ and $\operatorname{deg} \sigma \eta \leq 4$.

\section{A.4 Case A, Part I}

Assume that $\sigma$ has a zero at $z=s$ so that $\sigma(z)=(z-s) \rho(z)$. Clearly, $\operatorname{deg} \rho \leq 2$.

We make the ansatz

$$
c(z):=m(\lambda-s) \rho(z),
$$

where $m$ is a function only of $t$.

The equations (A.7) and (A.12) can be rewritten as

$$
\begin{aligned}
& \dot{\lambda}=m\left(2 \sigma(\lambda) \mu-(\lambda-s) \rho^{\prime}(\lambda)+\tau(\lambda)\right), \\
& \dot{\mu}=-m\left(\eta^{\prime}(\lambda)+\mu\left(\tau^{\prime}(\lambda)-\rho^{\prime}(\lambda)-(\lambda-s) \rho^{\prime \prime}(\lambda)\right)+\mu^{2} \sigma^{\prime}(\lambda)\right) .
\end{aligned}
$$

It is easy to check that (A.17) and (A.18) are the Hamilton equations for the Hamiltonian

$$
H=m\left(\eta(\lambda)+\left(\tau(\lambda)-(\lambda-s) \rho^{\prime}(\lambda)\right) \mu+\sigma(\lambda) \mu^{2}\right) .
$$

Another consequence of (A.16) is

$$
\frac{c \tau}{\sigma}(z)=\frac{m(\lambda-s) \tau(z)}{(z-s)}
$$


Therefore, using (A.4) we obtain

$$
\frac{\frac{c \tau}{\sigma}(z)-\frac{c \tau}{\sigma}(\lambda)-(z-\lambda)\left(\frac{c \tau}{\sigma}\right)^{\prime}(z)}{(z-\lambda)^{2}}=-\frac{m \tau(s)}{(z-s)^{2}} .
$$

Hence,

$$
I=\partial_{t} \frac{\tau}{\sigma}(z)-\frac{m \tau(s)}{(z-s)^{2}}
$$

Using

$$
\frac{c \sigma^{\prime}}{\sigma}(z)-c^{\prime}(z)=\frac{m(\lambda-s) \rho(z)}{z-s}
$$

we obtain

$$
\begin{gathered}
\frac{\eta(z)-\eta(\lambda)}{(z-\lambda)}\left(\frac{c \sigma^{\prime}}{\sigma}(z)-c^{\prime}(z)\right)-\eta^{\prime}(\lambda)\left(\frac{\sigma^{\prime} c}{\sigma}(\lambda)-c^{\prime}(\lambda)\right) \\
=\frac{m(\eta(z)-\eta(\lambda))(\lambda-s) \rho(z)}{(z-\lambda)(z-s)}-m \eta^{\prime}(\lambda) \rho(\lambda) .
\end{gathered}
$$

Therefore,

$$
\begin{aligned}
I I= & -\frac{\dot{\sigma}}{\sigma}(z)(\eta(z)-\eta(\lambda))+\dot{\eta}(z)-\dot{\eta}(\lambda)+\frac{m(\eta(z)-\eta(\lambda))(\lambda-s) \rho(z)}{(z-\lambda)(z-s)}-m \eta^{\prime}(\lambda) \rho(\lambda) \\
& +\frac{m(\lambda-s)}{(z-\lambda)^{2}}\left(2 \rho \eta(z)-2 \rho \eta(\lambda)-\left((\rho \eta)^{\prime}(z)+(\rho \eta)^{\prime}(\lambda)\right)(z-\lambda)\right) .
\end{aligned}
$$

Finally, we have

$$
\frac{c \sigma^{\prime}(z)}{\sigma(z)}=\frac{m(\lambda-s)\left(\rho(z)+(z-s) \rho^{\prime}(z)\right)}{(z-s)} .
$$

Using (A.4) with $\xi(z)=-\rho(z)-(z-s) \rho^{\prime}(z)$ and the interchanged role of $\lambda$ and $z$ we obtain

$$
\frac{\frac{c \sigma^{\prime}}{\sigma}(z)-\frac{c \sigma^{\prime}}{\sigma}(\lambda)-\left(\frac{c \sigma^{\prime}}{\sigma}\right)^{\prime}(\lambda)(z-\lambda)}{(z-\lambda)}=m \rho(s) \frac{(z-\lambda)}{(z-s)(\lambda-s)} .
$$

Therefore,

$$
I I I=\frac{\dot{\sigma}}{\sigma}(z)-\frac{\dot{\sigma}}{\sigma}(\lambda)-m \rho(s) \frac{(z-\lambda)}{(z-s)(\lambda-s)} .
$$

Subcase A1. We assume that the root $s$ is single and the time variable is chosen as $t=s$. We can write $\sigma(z)=(z-t) \rho(z), \rho(t) \neq 0, \operatorname{deg} \rho \leq 2$.

We assume $\operatorname{deg} \eta_{0} \leq 1, \operatorname{deg} \phi \leq 1, \kappa, \alpha \in \mathbb{C}$,

$$
\tau(z)=(1-\kappa) \rho(z)+\phi(z)(z-t), \quad \eta(z)=\frac{\alpha \rho(t)}{z-t}+\eta_{0}(z), \quad \dot{\kappa}=\dot{\rho}=\dot{\phi}=\dot{\eta}_{0}=\dot{\alpha}=0 .
$$

We have

$$
\partial_{t} \frac{\tau}{\sigma}(z)=\frac{1-\kappa}{(z-t)^{2}}, \quad 1-\kappa=\frac{\tau(t)}{\rho(t)} .
$$


Therefore,

$$
I=\frac{1-\kappa}{(z-t)^{2}}(1-m \rho(t)) .
$$

Using $\operatorname{deg} \eta_{0} \leq 1$ and $\dot{\eta}_{0}=0$ we obtain

$$
\begin{aligned}
& -\frac{\dot{\sigma}}{\sigma}(z)\left(\eta_{0}(z)-\eta_{0}(\lambda)\right)+\dot{\eta}_{0}(z)-\dot{\eta}_{0}(\lambda)=\frac{1}{(z-t)} \eta_{0}^{\prime}(z-\lambda), \\
& \frac{m\left(\eta_{0}(z)-\eta_{0}(\lambda)\right)(\lambda-t) \rho(z)}{(z-\lambda)(z-t)}-m \eta_{0}^{\prime}(\lambda) \rho(\lambda)=-\frac{m \rho(t) \eta_{0}^{\prime}(z-\lambda)}{(z-t)}+m \eta_{0}^{\prime}(\lambda-t) \frac{\rho^{\prime \prime}}{2}(z-\lambda) .
\end{aligned}
$$

Using $\operatorname{deg} \eta_{0} \rho \leq 3$ and (A.5) we get

$$
\begin{gathered}
\frac{m(\lambda-t)}{(z-\lambda)^{2}}\left(2 \rho \eta_{0}(z)-2 \rho \eta_{0}(\lambda)-\left(\left(\rho \eta_{0}\right)^{\prime}(z)+\left(\rho \eta_{0}\right)^{\prime}(\lambda)\right)(z-\lambda)\right) \\
=-\frac{m(\lambda-t)\left(\rho \eta_{0}\right)^{\prime \prime \prime}(z-\lambda)}{6}=-\frac{m(\lambda-t) \rho^{\prime \prime} \eta_{0}^{\prime}(z-\lambda)}{2} .
\end{gathered}
$$

Set $\psi(z):=\frac{\rho(t)}{z-t}$. We have

$$
\begin{aligned}
-\frac{\dot{\sigma}}{\sigma}(z)(\psi(z)-\psi(\lambda))+\dot{\psi}(z)-\dot{\psi}(\lambda)= & \left(\frac{\rho(t)}{z-t}+\rho^{\prime}(t)\right)\left(\frac{1}{z-t}-\frac{1}{\lambda-t}\right) \\
& +\rho(t)\left(\frac{1}{(z-t)^{2}}-\frac{1}{(\lambda-t)^{2}}\right), \\
\frac{m(\psi(z)-\psi(\lambda))(\lambda-t) \rho(z)}{(z-\lambda)(z-t)}-m \psi^{\prime}(\lambda) \rho(\lambda)= & -\frac{m \rho(t)}{(z-t)^{2}}\left(\rho(t)+\rho^{\prime}(t)(z-t)+\frac{\rho^{\prime \prime}}{2}(z-t)^{2}\right) \\
& +\frac{m \rho(t)}{(\lambda-t)^{2}}\left(\rho(t)+\rho^{\prime}(t)(\lambda-t)+\frac{\rho^{\prime \prime}}{2}(\lambda-t)^{2}\right) .
\end{aligned}
$$

Using (A.6) we get

$$
\begin{aligned}
\frac{m(\lambda-t)}{(z-\lambda)^{2}}\left(2 \rho \psi(z)-2 \rho \psi(\lambda)-\left((\rho \psi)^{\prime}(z)+(\rho \psi)^{\prime}(\lambda)\right)(z-\lambda)\right) & =\frac{m \rho(t)^{2}(z-\lambda)}{(z-t)^{2}(\lambda-t)} \\
& =-\frac{m \rho(t)^{2}}{(z-t)}\left(\frac{1}{z-t}-\frac{1}{\lambda-t}\right) .
\end{aligned}
$$

Combining the above identities we obtain

$$
\begin{aligned}
I I= & \left(\left(-(\lambda-t) \eta_{0}^{\prime}+\frac{\rho(t)}{z-t}+\rho^{\prime}(t)\right)\left(\frac{1}{z-t}-\frac{1}{\lambda-t}\right)+\rho(t)\left(\frac{1}{(z-t)^{2}}-\frac{1}{\lambda-t)^{2}}\right)\right) \\
& \times(1-m \rho(t)) .
\end{aligned}
$$

We have

$$
\frac{\dot{\sigma}}{\sigma}(z)-\frac{\dot{\sigma}}{\sigma}(\lambda)=-\frac{1}{z-t}+\frac{1}{\lambda-t}=\frac{z-\lambda}{(\lambda-t)(z-t)} .
$$

Therefore,

$$
I I I=\frac{z-\lambda}{(\lambda-t)(z-t)}(1-m \rho(t)) .
$$

Thus $m=\frac{1}{\rho(t)}$ implies $I=I I=I I I=0$. 


\section{A.5 Case A, Part II}

Assume that the root $s$ is at least double. Then $\rho(s)=0$ so that the normalization $m=\frac{1}{\rho(s)}$ does not work. We have to change the time variable.

Thus we assume $\sigma(z)=(z-s)^{2} \rho_{1}(z)$, where $\operatorname{deg} \rho_{1} \leq 1$. We also assume $\dot{s}=\dot{\rho}_{1}=0$. Then we have

$$
\begin{aligned}
& I=\frac{\dot{\tau}}{\sigma}(z)-\frac{m \tau(s)}{(z-s)^{2}} \\
& I I=\dot{\eta}(z)-\dot{\eta}(\lambda)+\frac{m(\lambda-s)}{(z-\lambda)}\left(\rho_{1} \eta(z)-\rho_{1} \eta(\lambda)-\left(\rho_{1} \eta\right)^{\prime}(\lambda)(z-\lambda)-\frac{(\rho \eta)^{\prime \prime \prime}}{6}(z-\lambda)^{2}\right) .
\end{aligned}
$$

We consider separately two subcases: in the first the time variable is contained in $p_{0}$ and in the second in $q_{0}$.

Subcase Ap. We assume that $\dot{\tau}_{0}=\dot{\eta}=0, \operatorname{deg} \tau_{0} \leq 2, \sigma \eta(s)=(\sigma \eta)^{\prime}(s)=0$,

$$
\tau(z)=\tau_{0}(z)+t \rho_{1}(z), \quad \tau_{0}(s) \neq 0 \quad \text { or } \quad \rho_{1}(s) \neq 0 .
$$

We have

$$
\frac{\dot{\tau}}{\sigma}(z)=\frac{1}{(z-s)^{2}}, \quad \tau(s)=\tau_{0}(s)+t \rho_{1}(s) \not \equiv 0 .
$$

Hence,

$$
I=\frac{1}{(z-s)^{2}}\left(1-m\left(\tau_{0}(s)+t \rho_{1}(s)\right)\right) .
$$

Thus $m=\left(\tau_{0}(s)+t \rho_{1}(s)\right)^{-1}$ implies $I=0$.

$\sigma \eta(s)=(\sigma \eta)^{\prime}(s)=0$ implies that $\rho_{1} \eta$ is a polynomial of degree $\leq 2$. Therefore, $(\rho \eta)^{\prime \prime \prime}=$ $\left.\left(\rho_{1} \eta\right)(z-s)\right)^{\prime \prime \prime}=3\left(\rho_{1} \eta\right)^{\prime \prime}$ and

$$
I I=\frac{m(\lambda-s)}{(z-\lambda)}\left(\rho_{1} \eta(z)-\rho_{1} \eta(\lambda)-\left(\rho_{1} \eta\right)^{\prime}(\lambda)(z-\lambda)-\frac{\left(\rho_{1} \eta\right)^{\prime \prime}}{2}(z-\lambda)^{2}\right)=0 .
$$

Subcase Aq. We assume that $\tau(s)=0, \sigma \eta_{0}(s)=0, \operatorname{deg} \sigma \eta_{0} \leq 4$,

$$
\begin{aligned}
& \eta(z)=\frac{t}{z-s}+\eta_{0}(z), \quad \dot{\sigma}=\dot{\tau}=\dot{\eta}_{0}=0, \\
& \rho_{1}(s) \neq 0 \quad \text { or } \quad\left(\sigma \eta_{0}\right)^{\prime} \neq 0 .
\end{aligned}
$$

Clearly, $I=0$.

Now

$$
\sigma \eta_{0}(z)=(z-s)\left(\sigma \eta_{0}\right)^{\prime}(s)+(z-s)^{2} \psi(z),
$$

where $\operatorname{deg} \psi \leq 2$. Therefore,

$$
\begin{aligned}
& \eta_{0} \rho_{1}(z)=\frac{\left(\sigma \eta_{0}\right)^{\prime}(s)}{z-s}+\psi(z) \\
& \rho_{1} \eta(z)=\frac{\left(\sigma \eta_{0}\right)^{\prime}(s)+\rho_{1}(s) t}{z-s}+\psi(z)+\rho_{1}^{\prime} t .
\end{aligned}
$$

Now $(\eta \rho)^{\prime \prime \prime}=\left((z-s) \rho_{1} \eta\right)^{\prime \prime \prime}=((z-s) \psi)^{\prime \prime \prime}=3 \psi^{\prime \prime}$. Therefore,

$$
\rho_{1} \eta(z)-\rho_{1} \eta(\lambda)-\left(\rho_{1} \eta\right)^{\prime}(\lambda)(z-\lambda)-\frac{(\rho \eta)^{\prime \prime \prime}}{6}(z-\lambda)^{2}
$$




$$
\begin{aligned}
= & \psi(z)-\psi(\lambda)-(z-\lambda) \psi^{\prime}(\lambda)-(z-\lambda)^{2} \frac{\psi^{\prime \prime}}{2} \\
& +\left(\left(\sigma \eta_{0}\right)^{\prime}(s)+\rho_{1}(s) t\right)\left(\frac{1}{z-s}-\frac{1}{\lambda-s}+\frac{(z-\lambda)}{(\lambda-s)^{2}}\right) .
\end{aligned}
$$

(A.19) vanishes, because $\operatorname{deg} \psi \leq 2$. Using (A.20), (A.3) and

$$
\dot{\eta}(z)-\dot{\eta}(\lambda)=\frac{1}{z-s}-\frac{1}{\lambda-s}
$$

we obtain

$$
I I=\left(\frac{1}{z-s}-\frac{1}{\lambda-s}\right)\left(1-m\left(\left(\sigma \eta_{0}\right)^{\prime}(s)+\rho_{1}(s) t\right)\right),
$$

where $\left(\sigma \eta_{0}\right)^{\prime}(s)+\rho_{1}(s) t \not \equiv 0$. Hence if $m=\left(\left(\sigma \eta_{0}\right)^{\prime}(s)+\rho_{1}(s) t\right)^{-1}$, then $I I=0$.

\section{A.6 Case B}

We assume that $\operatorname{deg} \sigma \leq 2, \operatorname{deg} \sigma \eta \leq 3$ and $\dot{\sigma}=0$. We set

$$
c(z)=m \sigma(z),
$$

where $m$ is a function just of $t$. The equations (A.7) and (A.12) can be rewritten as

$$
\begin{aligned}
& \dot{\lambda}=m\left(2 \sigma(\lambda) \mu-\sigma^{\prime}(\lambda)+\tau(\lambda)\right), \\
& \dot{\mu}=-m\left(\eta^{\prime}(\lambda)+\mu\left(\tau^{\prime}(\lambda)-\sigma^{\prime \prime}(\lambda)\right)+\mu^{2} \sigma^{\prime}(\lambda)\right) .
\end{aligned}
$$

We easily check that (A.21) and (A.22) are the Hamilton equations for the Hamiltonian

$$
H(t, \lambda, \mu):=m\left(\eta(\lambda)+\left(\tau(\lambda)-\sigma^{\prime}(\lambda)\right) \mu+\sigma(\lambda) \mu^{2}\right) .
$$

Using $\operatorname{deg} \tau \leq 2$, we get

$$
\frac{\tau(z)-\tau(\lambda)-(z-\lambda) \tau^{\prime}(z)}{(z-\lambda)^{2}}=-\frac{\tau^{\prime \prime}}{2} .
$$

Therefore,

$$
I=\frac{\dot{\tau}(z)}{\sigma(z)}-m \frac{\tau^{\prime \prime}}{2}
$$

Using $\operatorname{deg} \sigma \eta \leq 3$ we obtain

$$
\frac{2 \sigma \eta(z)-2 \sigma \eta(\lambda)-\left((\sigma \eta)^{\prime}(z)+(\sigma \eta)^{\prime}(\lambda)\right)(z-\lambda)}{(z-\lambda)^{2}}=-\frac{(\sigma \eta)^{\prime \prime \prime}}{6}(z-\lambda) .
$$

Therefore,

$$
I I=\dot{\eta}(z)-\dot{\eta}(\lambda)-m \frac{(\sigma \eta)^{\prime \prime \prime}}{6}(z-\lambda) .
$$

$I I I$ is clearly 0 , because $\dot{\sigma}=0$ and $\frac{c \sigma^{\prime}}{\sigma}$ is a polynomial of degree $\leq 1$.

This ends the proof of Theorem 4.3B.

Again, we consider two subcases, with $t$ contained in $p_{0}$ and in $q_{0}$. 
Subcase Bp. $\operatorname{deg} \sigma \leq 2, \operatorname{deg} \tau_{0} \leq 2, \operatorname{deg} \sigma \eta \leq 2$,

$$
\begin{aligned}
& \tau(z)=t \sigma(z)+\tau_{0}(z), \quad \dot{\sigma}=\dot{\tau}_{0}=\dot{\eta}=0, \\
& \operatorname{deg} \sigma=2 \quad \text { or } \quad \operatorname{deg} \tau_{0}=2 .
\end{aligned}
$$

$I I=0$ is automatic. Moreover, $\tau^{\prime \prime}=t \sigma^{\prime \prime}+\tau_{0}^{\prime \prime} \not \equiv 0$ and

$$
I=1-m\left(t \frac{\sigma^{\prime \prime}}{2}+\frac{\tau^{\prime \prime}}{2}\right)
$$

Hence $m=\left(t \frac{\sigma^{\prime \prime}}{2}+\frac{\tau_{0}^{\prime \prime}}{2}\right)^{-1}$ implies $I=0$.

Subcase Bq. $\operatorname{deg} \sigma \leq 2, \operatorname{deg} \tau \leq 1, \operatorname{deg} \sigma \eta_{0} \leq 3$,

$$
\begin{array}{ll}
\eta(z)=t z+\eta_{0}(z), & \dot{\sigma}=\dot{\tau}=\dot{\eta}_{0}=0 \\
\operatorname{deg} \sigma=2 \quad \text { or } & \operatorname{deg} \sigma \eta_{0}=3 .
\end{array}
$$

$I=0$ is automatic. Moreover, $(\sigma \eta)^{\prime \prime \prime}=t 3 \sigma^{\prime \prime}+(\sigma \eta)^{\prime \prime \prime} \not \equiv 0$ and

$$
I I=(z-\lambda)\left(1-m\left(t \frac{\sigma^{\prime \prime}}{2}+\frac{\left(\sigma \eta_{0}\right)^{\prime \prime \prime}}{6}\right)\right)
$$

Therefore $m=\left(t \frac{\sigma^{\prime \prime}}{2}+\frac{\left(\sigma \eta_{0}\right)^{\prime \prime \prime}}{6}\right)^{-1}$ implies $I I=0$.

\section{B Hamilton equations}

\section{B.1 From Hamilton equations to second order equations}

We devote this appendix to a few remarks about Hamilton equations.

Suppose that $H(t, \lambda, \mu)$ is a time-dependent Hamiltonian. The equations

$$
\begin{aligned}
& \frac{\mathrm{d} \lambda}{\mathrm{d} t}=\frac{\partial H}{\partial \mu}(t, \lambda, \mu), \\
& \frac{\mathrm{d} \mu}{\mathrm{d} t}=-\frac{\partial H}{\partial \lambda}(t, \lambda, \mu),
\end{aligned}
$$

are called the Hamilton equations generated by $H$.

All Painlevé Hamiltonians have the form

$$
H(t, \lambda, \mu)=f(t, \lambda) \frac{\mu^{2}}{2}+\mu g(t, \lambda)+h(t, \lambda) .
$$

For such Hamiltonians it is easy to eliminate $\mu$ from the Hamilton equations. One obtains a second order differential equation for $\lambda$ of the form

$$
\begin{aligned}
& \frac{\mathrm{d}^{2} \lambda}{\mathrm{d} t^{2}}=A(t, \lambda)\left(\frac{\mathrm{d} \lambda}{\mathrm{d} t}\right)^{2}+B(t, \lambda) \frac{\mathrm{d} \lambda}{\mathrm{d} t}+C(t, \lambda), \\
& A:=\frac{1}{2 f} \frac{\partial f}{\partial \lambda}, \quad B:=\frac{1}{f} \frac{\partial f}{\partial t}, \quad C:=-\frac{g^{2}}{2 f} \frac{\partial f}{\partial \lambda}-\frac{g}{f} \frac{\partial f}{\partial t}+g \frac{\partial g}{\partial \lambda}+\frac{\partial g}{\partial t}-f \frac{\partial h}{\partial \lambda} .
\end{aligned}
$$




\section{B.2 Invariance of Hamilton equations}

The Hamilton equations are invariant with respect to various transformations.

- The equations generated by $\epsilon H(\epsilon t, \lambda, \mu)$ are equivalent to (B.1) and (B.2).

- Let $(\lambda, \mu) \mapsto(\tilde{\lambda}, \tilde{\mu})$ be a (time-independent) canonical transformation, that means

$$
\frac{\partial \tilde{\lambda}}{\partial \lambda} \frac{\partial \tilde{\mu}}{\partial \mu}-\frac{\partial \tilde{\lambda}}{\partial \mu} \frac{\partial \tilde{\mu}}{\partial \lambda}=1
$$

Then the Hamilton equations in the new variables

$$
\frac{\mathrm{d} \tilde{\lambda}}{\mathrm{d} t}=\frac{\partial H}{\partial \tilde{\mu}}, \quad \frac{\mathrm{d} \tilde{\mu}}{\mathrm{d} t}=-\frac{\partial H}{\partial \tilde{\lambda}},
$$

are equivalent to (B.2).

- The Hamilton equations are invariant with respect to the following time-dependent transformation:

$$
\tilde{\lambda}=t^{-1} \lambda, \quad \tilde{\mu}=t \mu, \quad \tilde{H}=H-t^{-1} \tilde{\lambda} \tilde{\mu} .
$$

\section{B.3 Hamiltonian solvable in quadratures}

Let us now consider a Hamiltonian of the form

$$
H(t, \lambda, \mu)=m(t)\left(f(\lambda) \frac{\mu^{2}}{2}+\mu g(\lambda)+h(\lambda)\right) .
$$

We will show that it is solvable in quadratures.

First we change the time from $t$ to $s$, by solving

$$
\frac{\mathrm{d} s}{\mathrm{~d} t}=m(t)
$$

Using the time $s$ we can replace (B.3) by the time-independent Hamiltonian

$$
H(\lambda, \mu)=f(\lambda) \frac{\mu^{2}}{2}+\mu g(\lambda)+h(\lambda) .
$$

Now

$$
E:=f(\lambda) \frac{\mu^{2}}{2}+\mu g(\lambda)+h(\lambda)
$$

is a constant of motion, hence we can express $\mu$ in terms of $\lambda$ :

$$
\mu=\frac{-g(\lambda) \pm \sqrt{g(\lambda)^{2}-2 f(\lambda)(h(\lambda)-E)}}{f(\lambda)} .
$$

We insert this into the first Hamilton equation

$$
\frac{\mathrm{d} \lambda}{\mathrm{d} s}=\mu f(\lambda)+g(\lambda)
$$

obtaining

$$
\frac{\mathrm{d} \lambda}{\mathrm{d} s}= \pm \sqrt{g(\lambda)^{2}-2 f(\lambda)(h(\lambda)-E)} .
$$

This is clearly solvable in quadratures. 


\section{Acknowledgements}

J.D. and A.I. would like to express their gratitude to Galina Filipuk for very useful discussions and remarks. A.I. acknowledges the support by the Armenian Science Committee (SC Grant No. 20RF-171), and the Armenian National Science and Education Fund (ANSEF Grant No. PS5701). The work of J.D. and A.L. was supported by National Science Center (Poland) under the grant UMO-2019/35/B/ST1/01651.

\section{References}

[1] Chiang Y.-M., Law C.-K., Yu G.-F., Invariant subspaces of biconfluent Heun operators and special solutions of Painlevé IV, arXiv:1905.10046.

[2] Dereziński J., Hypergeometric type functions and their symmetries, Ann. Henri Poincaré 15 (2014), 15691653, arXiv:1305.3113.

[3] Dereziński J., Group-theoretical origin of symmetries of hypergeometric class equations and functions, in Complex Differential and Difference Equations (Proceedings of the School and Conference Held at Bedlewo, Poland, September 2-15, 2018), Editors G. Filipuk, A. Lastra, S. Michalik, Y. Takei, H. Żołądek, De Gruyter Proceedings in Mathematics, Berlin, 2020, 3-128, arXiv:1906.03512.

[4] Dereziński J., Wrochna M., Exactly solvable Schrödinger operators, Ann. Henri Poincaré 12 (2011), 397418, arXiv:1009.0541.

[5] Filipuk G., Ishkhanyan A., Dereziński J., On the derivatives of the Heun functions, J. Contemp. Math. Anal. 55 (2020), 200-207, arXiv:1907.12692.

[6] Fuchs R., Über lineare homogene Differentialgleichungen zweiter Ordnung mit drei im Endlichen gelegenen wesentlich singulären Stellen, Math. Ann. 63 (1907), 301-321.

[7] Gambier B., Sur les équations différentielles du second ordre et du premier degré dont l'intégrale générale est a points critiques fixes, Acta Math. 33 (1910), 1-55.

[8] Heun K., Zur Theorie der Riemann'schen Functionen zweiter Ordnung mit vier Verzweigungspunkten, Math. Ann. 33 (1888), 161-179.

[9] Ince E.L., Ordinary differential equations, Dover Publications, New York, 1944.

[10] Ishkhanyan A., Suominen K.-A., New solutions of Heun's general equation, J. Phys. A: Math. Gen. 36 (2003), L81-L85, arXiv:0909.1684.

[11] Its A.R., Prokhorov A., On some Hamiltonian properties of the isomonodromic tau functions, in Ludwig Faddeev Memorial Volume, World Sci. Publ., Hackensack, NJ, 2018, 227-264, arXiv:1803.04212.

[12] Iwasaki K., Kimura H., Shimomura S., Yoshida M., From Gauss to Painlevé. A modern theory of special functions, Aspects of Mathematics, Vol. E16, Friedr. Vieweg \& Sohn, Braunschweig, 1991.

[13] Jimbo M., Monodromy problem and the boundary condition for some Painlevé equations, Publ. Res. Inst. Math. Sci. 18 (1982), 1137-1161.

[14] Jimbo M., Miwa T., Monodromy preserving deformation of linear ordinary differential equations with rational coefficients. II, Phys. D 2 (1981), 407-448.

[15] Kimura T., On Fuchsian differential equations reducible to hypergeometric equations by linear transformations, Funkcial. Ekvac. 13 (1971), 213-232.

[16] Maier R.S., The 192 solutions of the Heun equation, Math. Comp. 76 (2007), 811-843, arXiv:math.CA/0408317.

[17] Mason P., Differential equations and singularities II, available at https://analyticphysics.com.

[18] Nikiforov A.F., Uvarov V.B., Special functions of mathematical physics. A unified introduction with applications, Birkhäuser Verlag, Basel, 1988.

[19] Ohyama Y., Okumura S., A coalescent diagram of the Painlevé equations from the viewpoint of isomonodromic deformations, J. Phys. A: Math. Gen. 39 (2006), 12129-12151, arXiv:math.CA/0601614.

[20] Okamoto K., Isomonodromic deformation and Painlevé equations, and the Garnier system, J. Fac. Sci. Univ. Tokyo Sect. IA Math. 33 (1986), 575-618.

[21] Painlevé P., Mémoire sur les équations différentielles dont l'intégrale générale est uniforme, Bull. Soc. Math. France 28 (1900), 201-261. 
[22] Painlevé P., Sur les équations différentielles du second ordre et d'ordre supérieur dont l'intégrale générale est uniforme, Acta Math. 25 (1902), 1-85.

[23] Poole E.G.C., Linear differential equations, Oxford University Press, Oxford, 1936.

[24] Ronveaux A. (Editor), Heun's differential equations, Oxford University Press, Oxford, 1995.

[25] Slavyanov S.Yu., Lay W., Special functions. A unified theory based on singularities, Oxford Mathematical Monographs, Oxford University Press, Oxford, 2000.

[26] Slavyanov S.Yu., Shatco D.A., Ishkhanyan A.M., Rotinyan T.A., Generation and removal of apparent singularities in linear ordinary differential equations with polynomial coefficients, Theoret. and Math. Phys. 189 (2016), 1726-1733, arXiv:1606.01476. 